\title{
Bottom-Up Synthesis of Optically Active Oligonaphthalenes: Three Different Pathways for Controlling the Axial Chirality.
}

Kazunori Tsubaki, ${ }^{* a}$ Hiroyuki Tanaka, ${ }^{a}$ Kazuto Takaishi, ${ }^{a}$ Masaya Miura, ${ }^{a}$ Hiroshi Morikawa, ${ }^{a}$ Takumi Furuta, ${ }^{\mathrm{b}}$ Kiyoshi Tanaka, ${ }^{\mathrm{b}}$ Kaoru Fuji, ${ }^{\mathrm{c}}$ Takahiro Sasamori, ${ }^{\mathrm{a}}$ Norihiro Tokitoh, ${ }^{\mathrm{a}}$ Takeo Kawabata $^{\text {a }}$

a) Institute for Chemical Research, Kyoto University, Gokasho, Uji, Kyoto, 611-0011, Japan.

b) School of Pharmaceutical Sciences, University of Shizuoka, Yada, Shizuoka, 422-8002, Japan.

c) Faculty of Pharmaceutical Sciences, Hiroshima International University, 5-1-1 Hirokoshingai, Kure, Hiroshima 737-0112, Japan

e-mail: tsubaki@fos.kuicr.kyoto-u.ac.jp

Page S2: $\quad$ UV and CD spectra of 22-25.

Pages S3-S43: Synthesis of the Oligonaphthalene Derivatives.

Page S44-S48: Synthesis of the Bispyrene Derivatives.

Page S49-S55: General Procedure for the Synthesis of ${ }^{13} \mathrm{C}$ enrich Compounds 29 - 31. 
UV and CD spectra of 22-25.

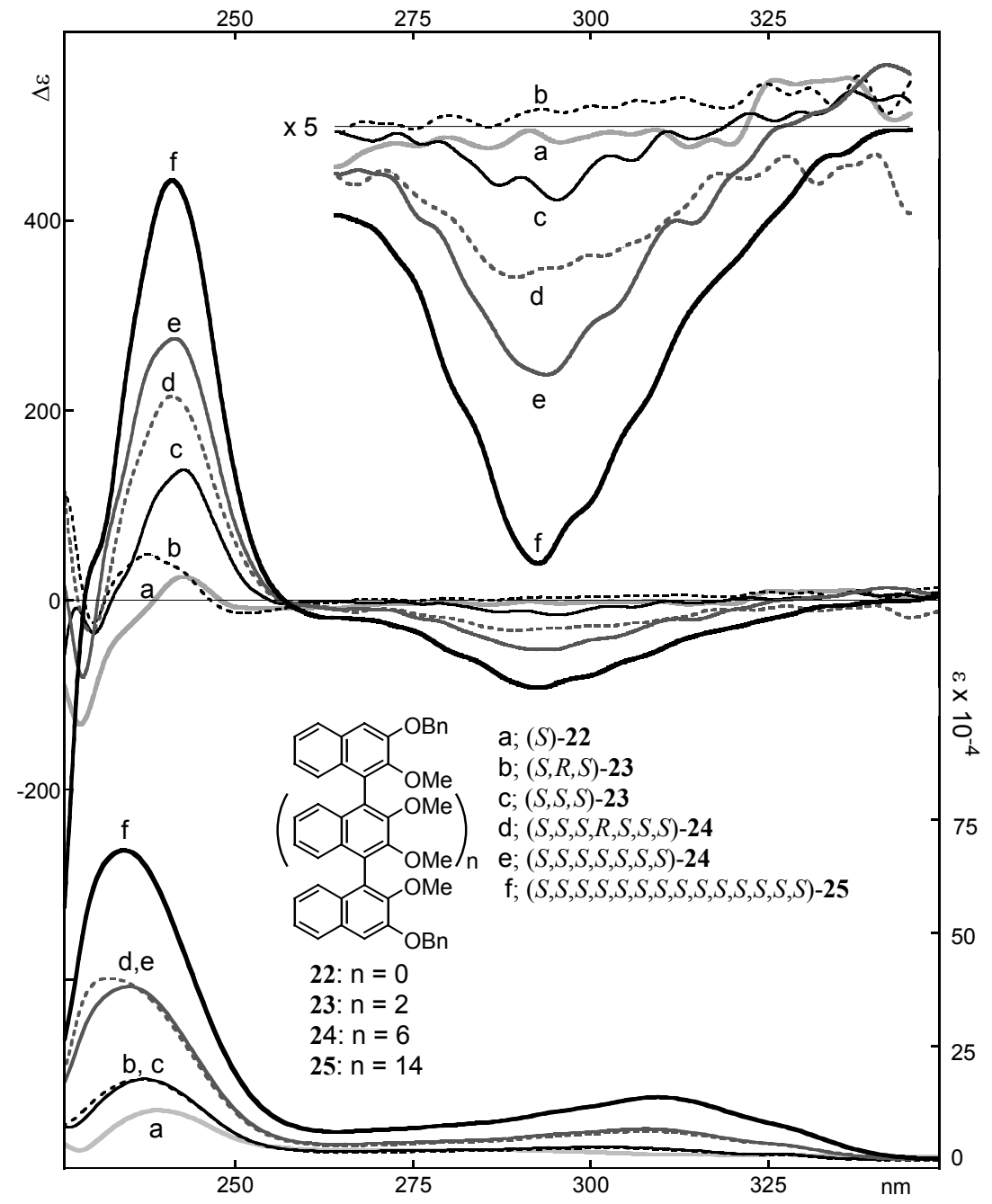

Condition of UV-vis spectra: Chloroform $1.0 \times 10^{-5} \mathrm{M}$, light path length $=1 \mathrm{~mm}, 20^{\circ} \mathrm{C}$.

Condition of CD spectra: Chloroform $1.0 \times 10^{-5} \mathrm{M}$, light path length $=1 \mathrm{~mm}, 25^{\circ} \mathrm{C}$.

\begin{tabular}{lcccc} 
oligonaphthalenes & $\lambda_{1}(\mathrm{~nm})$ & $\varepsilon_{1}{ }^{* *}$ & $\lambda_{2}(\mathrm{~nm})$ & $\varepsilon_{2}{ }^{* *}$ \\
\hline$(S)-\mathbf{2 2}$ & 239 & $109000(54500)$ & 279 & $21000(10500)$ \\
$(S, R, S)-\mathbf{2 3}$ & 237 & $178000(44500)$ & 304 & $26000(6500)$ \\
$(S, S, S)-\mathbf{2 3}$ & 237 & $178000(44500)$ & 300 & $28000(7000)$ \\
$(S, S, S, R, S, S, S)-\mathbf{2 4}$ & 232 & $400000(50000)$ & 308 & $63000(7900)$ \\
$(S, S, S, S, S, S, S)-\mathbf{2 4}$ & 235 & $382000(47800)$ & 308 & $68000(8500)$ \\
all- $(S)-\mathbf{2 5}^{*}$ & 235 & $682000(42600)$ & 310 & $138000(8600)$ \\
\hline
\end{tabular}

Condition: Chloroform $1.0 \times 10^{-5} \mathrm{M}$, light path length $=1 \mathrm{~mm}, 20{ }^{\circ} \mathrm{C}$.

* all-(S)-25; $(S, S, S, S, S, S, S, S, S, S, S, S, S, S, S)-\mathbf{2 5}, * *(\quad)=\varepsilon /$ [number of naphthalene units] 


\section{Experimental Section}

General: Unless otherwise specified, all ${ }^{1} \mathrm{H}-\mathrm{NMR}$ spectra were taken at 200 or $300 \mathrm{MHz}$ in $\mathrm{CDCl}_{3}$ with chemical shifts being reported at $\delta$ ppm from tetramethylsilane as an internal standard. Flash column chromatography was carried out with silica gel 60 spherical (150-325 mesh) and silica gel 60 $\mathrm{F}_{254}$ plates (Merck) for preparative TLC.

Scheme SI-1.

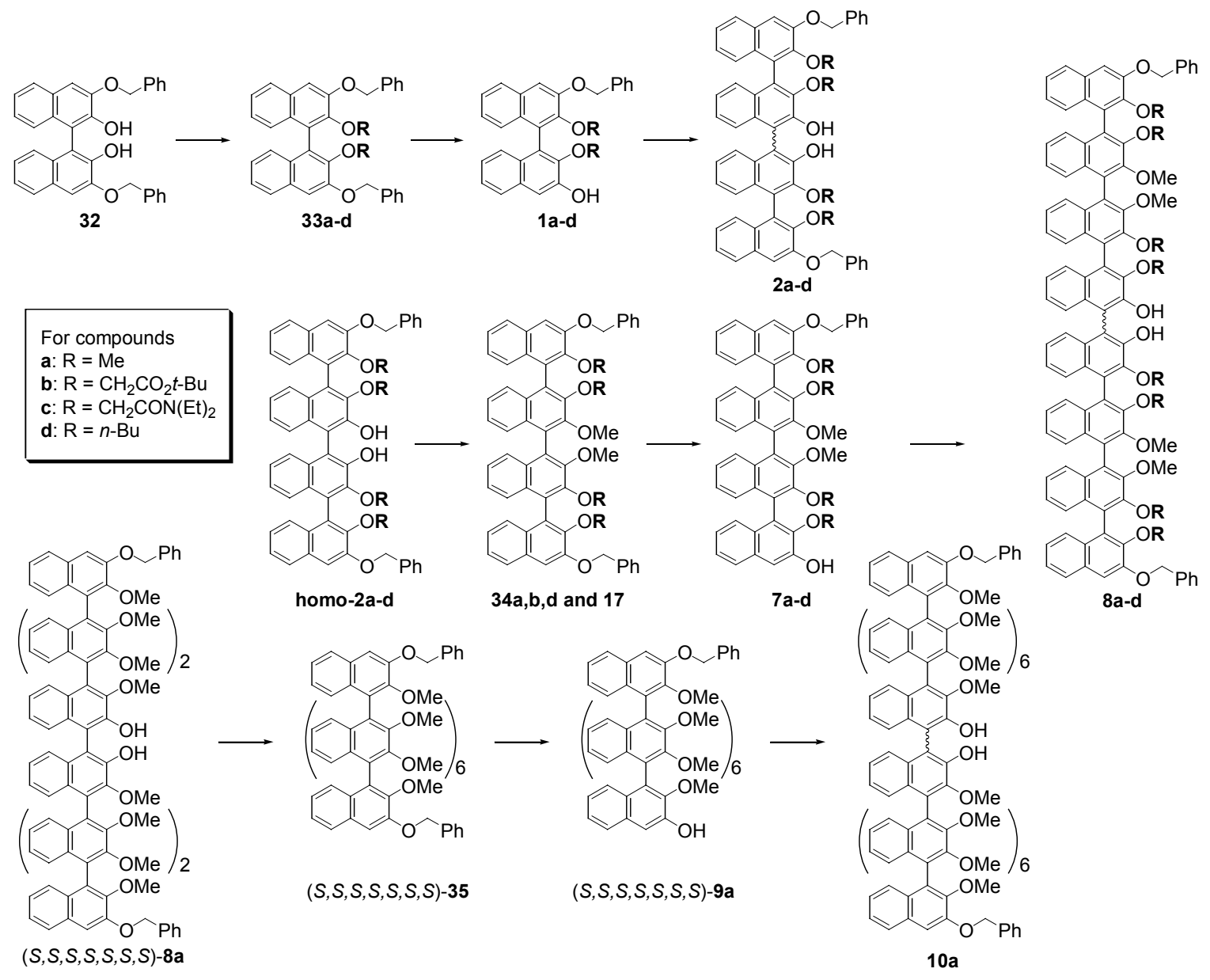

Compounds 32, 33a, 1a, 2a, 34a, 7a, 8a, 35, 9a and 10a are known.

Tsubaki, K.; Miura, M.; Morikawa, H.; Tanaka, H.; Kawabata, T.; Furuta, T.; Tanaka, K.; Fuji. K. J. Am. Chem. Soc. 2003, 125, 16200-16201. 
General Procedure for the Synthesis of Compounds 33b-d: The synthesis of $(R)-33 b$ is typical. Tert-butyl bromoacetate $(2.58 \mathrm{~g}, 1.96 \mathrm{ml}, 13.24 \mathrm{mmol}, 2.2$ eq.) was added to a suspension of $(R)-32(3.0$ g, $6.02 \mathrm{mmol})$ and $\mathrm{K}_{2} \mathrm{CO}_{3}(1.83 \mathrm{~g}, 13.24 \mathrm{mmol}, 2.2$ eq.) in DMF (10 ml). The suspension was stirred for 5 hours at room temperature. The reaction mixture was poured into the mixed solvent of ethyl acetate and hydrochloric acid solution. The organic layer was separated and washed successively with hydrochloric acid solution, water (twice) and brine. After dried over sodium sulfate, the solvent was evaporated in vacuo to afford crude of $(R)-33 \mathbf{b}$ as a white powder (4.19 g, 96\% yield). Crude $(R)-33 \mathbf{b}$ was directly used for the next step without further purification. A small part of the sample was subjected to further purification by PTLC to give an analytical sample.

$(R)-33 b: 96 \%$ yield; colorless viscous oil; $[\alpha]_{\mathrm{D}}^{20}=+11.8\left(\mathrm{c}=1.43, \mathrm{CHCl}_{3}\right)$; IR $(\mathrm{KBr}) 2979,1755$, $1725,1597 \mathrm{~cm}^{-1} ;{ }^{1} \mathrm{H}-\mathrm{NMR}\left(200 \mathrm{MHz}, \mathrm{CDCl}_{3}\right) \delta 1.20(\mathrm{~s}, 18 \mathrm{H}), 4.24(\mathrm{~d}, J=15.6,2 \mathrm{H}), 4.57(\mathrm{~d}, J=15.6$, 2H), $5.30(\mathrm{~s}, 4 \mathrm{H}), 7.0-7.6(\mathrm{~m}, 18 \mathrm{H}), 7.74(\mathrm{~d}, J=8.0,2 \mathrm{H})$; HRMS (EI) Calcd for $\mathrm{C}_{46} \mathrm{H}_{46} \mathrm{O}_{8}: 726.3193$. Found: 726.3197; Anal. Calcd for $\mathrm{C}_{46} \mathrm{H}_{46} \mathrm{O}_{8}$ : C, 76.01; H, 6.38. Found: C, 76.16; H, 6.44.

$(S)-33 c$ : $85 \%$ yield from $(S)-32$ and 2 -chloro- $N, N$-diethylacetamide; colorless foam; $[\alpha]_{\mathrm{D}}{ }^{20}=-14.5(\mathrm{c}$ $\left.=0.97, \mathrm{CHCl}_{3}\right) ; \mathrm{IR}(\mathrm{KBr}) 2972,1638,1436,1386,1230 \mathrm{~cm}^{-1} ;{ }^{1} \mathrm{H}-\mathrm{NMR}\left(200 \mathrm{MHz}, \mathrm{CDCl}_{3}\right) \delta 0.53(\mathrm{t}, J$ $=7.1,6 \mathrm{H}), 0.89(\mathrm{t}, J=7.1,6 \mathrm{H}), 2.5-2.8(\mathrm{~m}, 4 \mathrm{H}), 2.9-3.2(\mathrm{~m}, 4 \mathrm{H}), 4.34(\mathrm{~d}, J=12.0,2 \mathrm{H}), 4.62(\mathrm{~d}, J=$ 12.0, 2H), $5.32(\mathrm{~s}, 4 \mathrm{H}), 7.1-7.5(\mathrm{~m}, 16 \mathrm{H}), 7.58(\mathrm{~d}, J=6.2,2 \mathrm{H}), 7.75(\mathrm{~d}, J=8.0,2 \mathrm{H})$; HRMS (EI) Calcd for $\mathrm{C}_{46} \mathrm{H}_{48} \mathrm{~N}_{2} \mathrm{O}_{6}: 724.3513$. Found: 724.3536 .

$(S)-33 d$ :quantitative yield from $(S)-32$ and 1-bromobutane; pale yellow oil; $[\alpha]_{\mathrm{D}}{ }^{22}=-4.6(\mathrm{c}=0.50$, $\left.\mathrm{CHCl}_{3}\right)$; IR (neat) 2956, 1595, 1441, $1378 \mathrm{~cm}^{-1} ;{ }^{1} \mathrm{H}-\mathrm{NMR}\left(200 \mathrm{MHz}, \mathrm{CDCl}_{3}\right) \delta 0.45(\mathrm{t}, J=7.2,6 \mathrm{H})$, 0.6-0.9 (m, 4H), 1.1-1.3 (m, 4H), 3.6-3.7 (m, 2H), 3.9-4.1 (m, 2H), $5.31(\mathrm{~s}, 4 \mathrm{H}), 7.10-7.15(\mathrm{~m}, 4 \mathrm{H})$, 7.25-7.45 (m, 10H), $7.53(\mathrm{~d}, J=7.6,4 \mathrm{H}), 7.75(\mathrm{~d}, J=8.4,2 \mathrm{H})$; HRMS (EI) Calcd for $\mathrm{C}_{42} \mathrm{H}_{42} \mathrm{O}_{4}$ : 610.3083. Found: 610.3079; Anal. Calcd for $\mathrm{C}_{42} \mathrm{H}_{42} \mathrm{O}_{4} \cdot \mathrm{H}_{2} \mathrm{O}: \mathrm{C}, 80.22 ; \mathrm{H}, 7.05$. Found: C, 80.03; H, 6.84. 


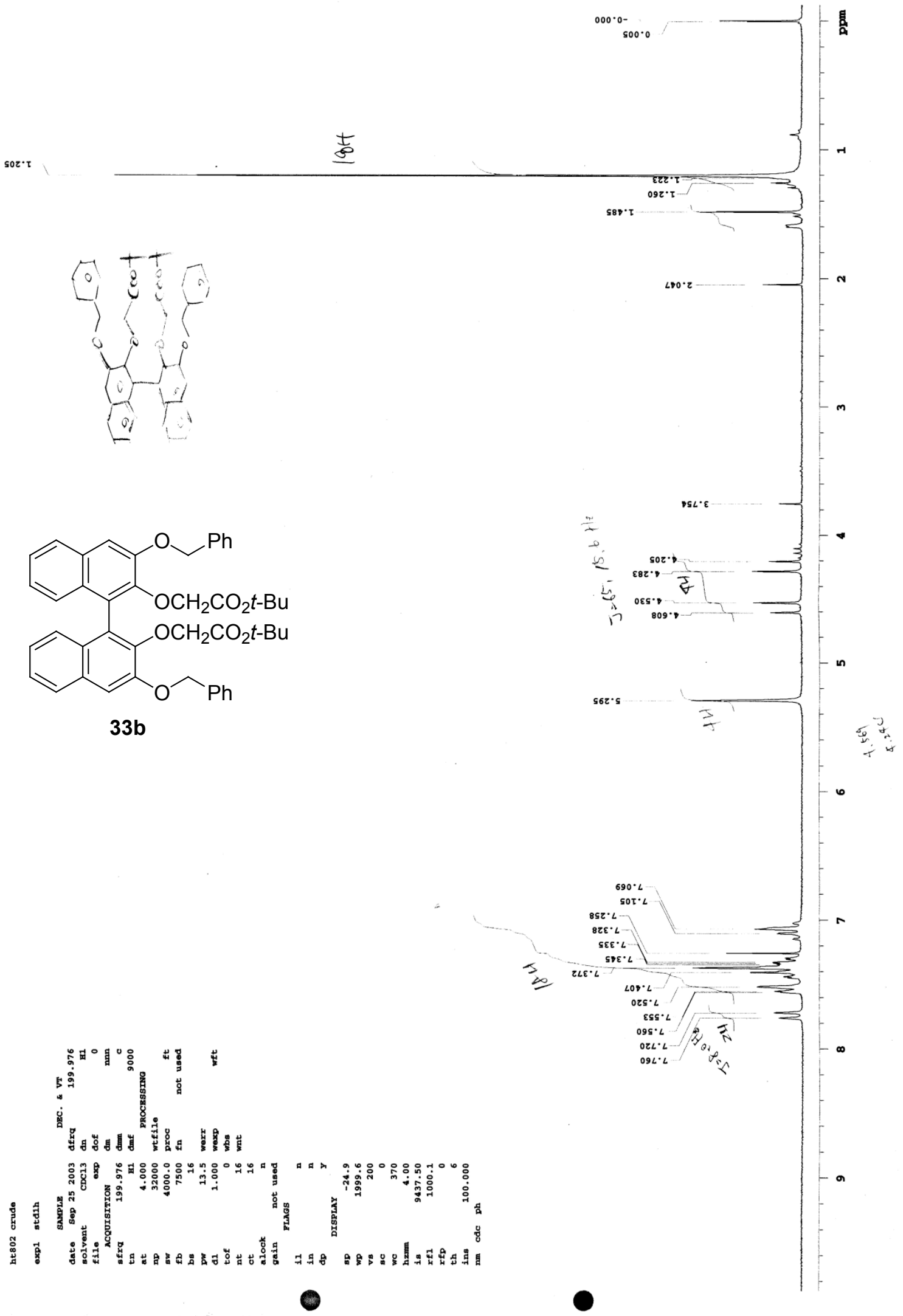




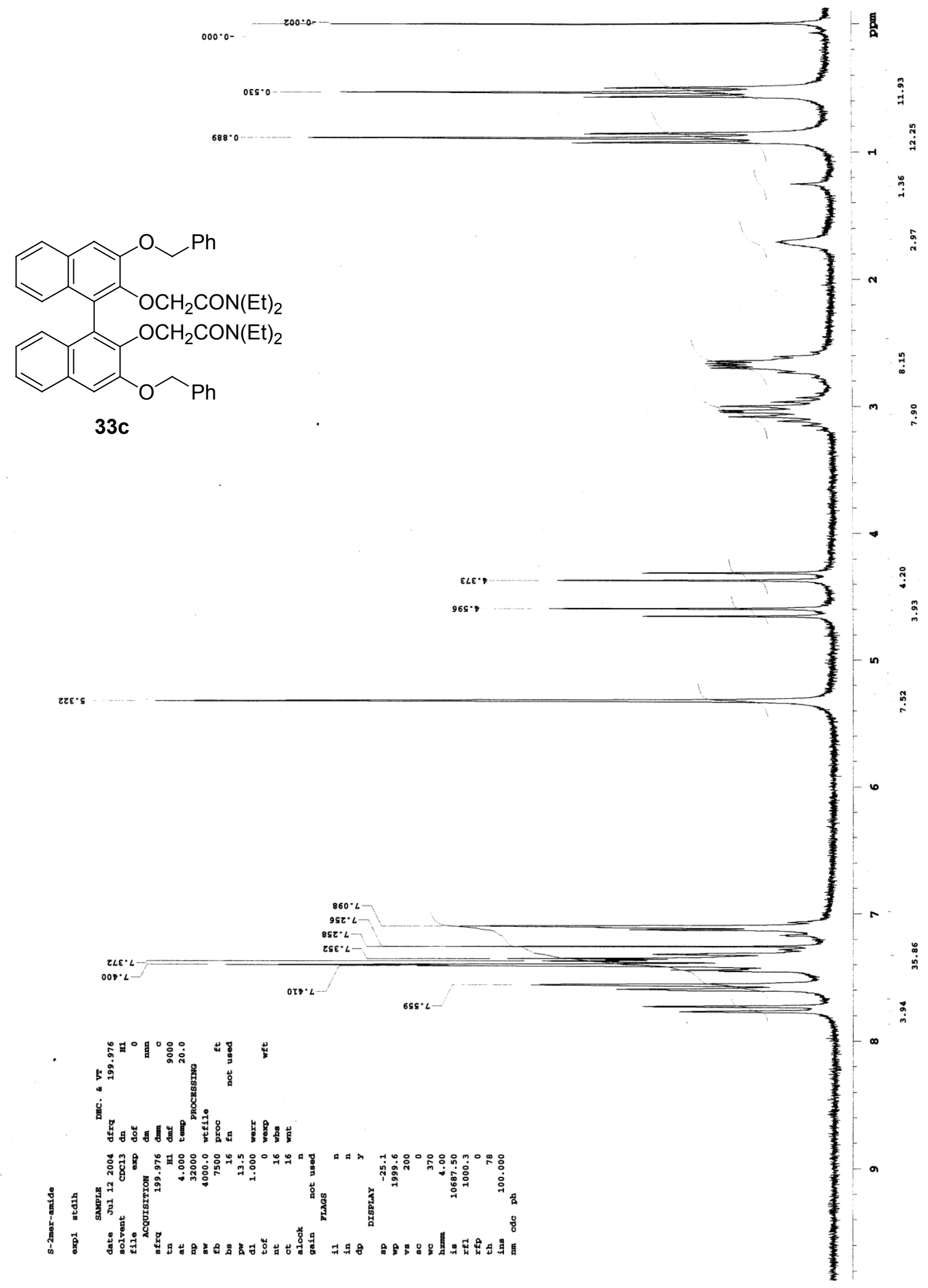




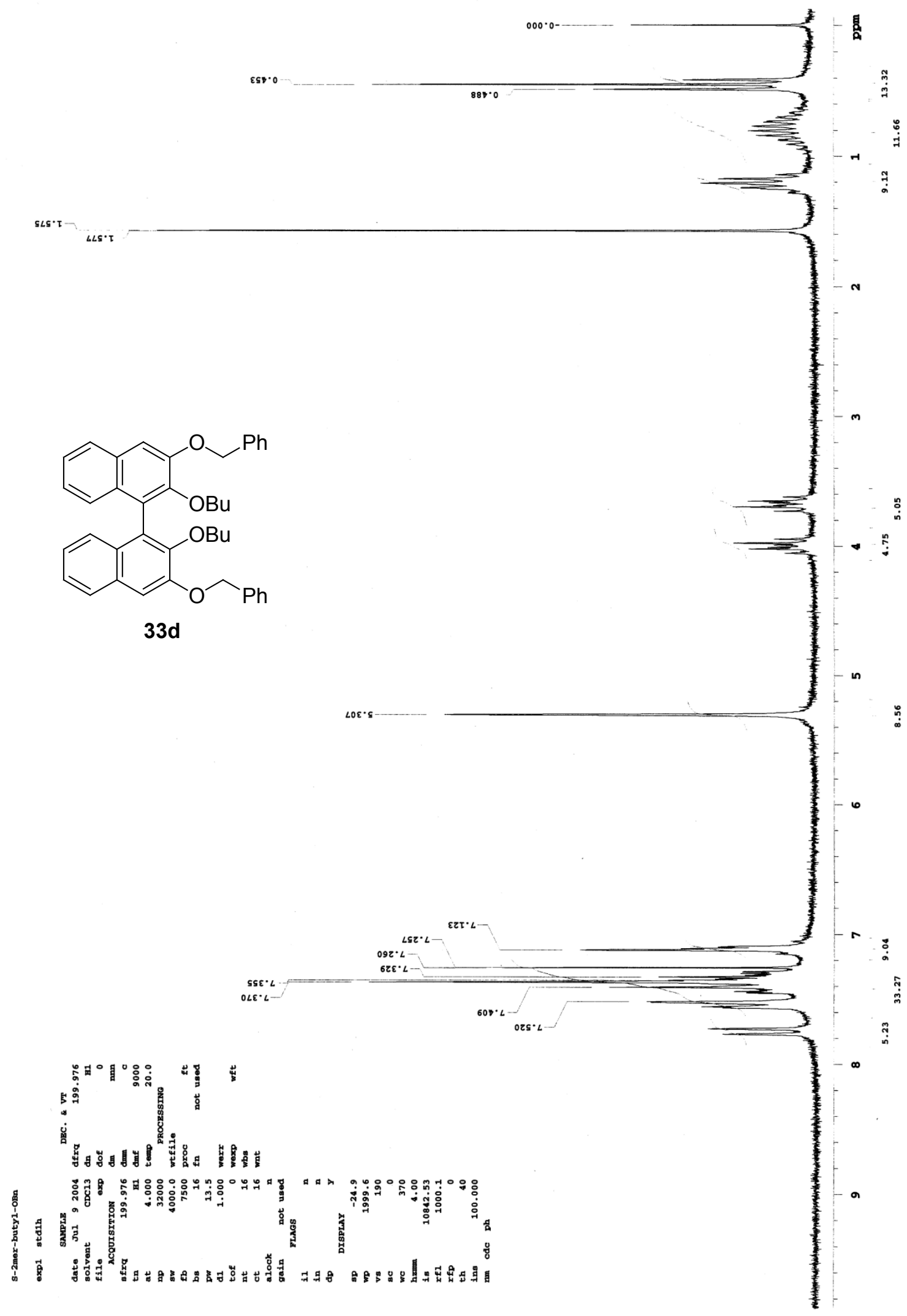


The synthesis of $(\boldsymbol{R})-1 \mathbf{b}:$ A suspension of $(R)-33 b(60.4 \mathrm{~g}, 80.2 \mathrm{mmol})$ and $10 \%$ palladium on carbon $(8.5 \mathrm{~g})$ in THF $(300 \mathrm{ml})$, was stirred under hydrogen atmosphere for 6 hours at room temperature with monitoring the reaction by TLC (hexane/ethyl acetate $=10 / 1$ x 5 times). After palladium on carbon was filtered off, the palladium on carbon was washed with chloroform and ethyl acetate. Organic layers and filtrate were combined and evaporated to give white solid. The white solid was filtered and recrystallized from ethyl acetate and hexane to give pure $(R)-\mathbf{1 b}(25.7 \mathrm{~g}, 50 \%$ yield). The filtrate was evaporated in vacuo to give a residue $(32.8 \mathrm{~g})$. The residue was purified by column chromatography $\left(\mathrm{SiO}_{2}\right.$, hexane/ethyl acetate $\left.=10 / 1\right)$ to afford $(R)-\mathbf{1 b}(16.2 \mathrm{~g}, 32 \%)$ and recovered $(R)-\mathbf{3 3 b}(8.3 \mathrm{~g})$.

$(R)-\mathbf{1 b}: \mathrm{Mp}, 144-146{ }^{\circ} \mathrm{C} ;[\alpha]_{\mathrm{D}}{ }^{20}=+3.1\left(\mathrm{c}=1.06, \mathrm{CHCl}_{3}\right) ; \mathrm{IR}(\mathrm{KBr}) 3265,1756,1724,1448,1241$ $\mathrm{cm}^{-1} ;{ }^{1} \mathrm{H}-\mathrm{NMR}\left(200 \mathrm{MHz}, \mathrm{CDCl}_{3}\right) \delta 1.19(\mathrm{~s}, 9 \mathrm{H}), 1.43(\mathrm{~s}, 9 \mathrm{H}), 3.96(\mathrm{~d}, J=17.0,1 \mathrm{H}), 4.07(\mathrm{~d}, J=15.4$, $1 \mathrm{H}), 4.28(\mathrm{~d}, J=17.0,1 \mathrm{H}), 4.60(\mathrm{~d}, J=15.4,1 \mathrm{H}), 5.32(\mathrm{~s}, 2 \mathrm{H}), 7.0-7.6(\mathrm{~m}, 13 \mathrm{H}), 7.7-7.8(\mathrm{~m}, 2 \mathrm{H}), 9.08$ (s, 1H); HRMS (EI) Calcd for $\mathrm{C}_{39} \mathrm{H}_{40} \mathrm{O}_{8}$ : 636.2723. Found: 636.2731; Anal. Calcd for $\mathrm{C}_{39} \mathrm{H}_{40} \mathrm{O}_{8} \cdot 0.5 \mathrm{H}_{2} \mathrm{O}: \mathrm{C}, 72.54 ; \mathrm{H}, 6.40$. Found: C, 72.30; H, 6.28.

(S)-1c: Similar to the above; $49 \%$ yield; white powder; Mp, $173-174{ }^{\circ} \mathrm{C} ;[\alpha]_{\mathrm{D}}{ }^{20}=-8.4(\mathrm{c}=1.00$, $\left.\mathrm{CHCl}_{3}\right)$; IR (KBr) 2973, 1639, 1498, 1438, $1246 \mathrm{~cm}^{-1} ;{ }^{1} \mathrm{H}-\mathrm{NMR}\left(200 \mathrm{MHz}, \mathrm{CDCl}_{3}\right) \delta 0.54(\mathrm{t}, J=7.1$, $3 \mathrm{H}), 0.83(\mathrm{t}, J=7.1,3 \mathrm{H}), 0.90(\mathrm{t}, J=6.9,3 \mathrm{H}), 1.06(\mathrm{t}, J=7.0,3 \mathrm{H}), 2.59(\mathrm{q}, J=7.4,2 \mathrm{H}), 2.7-2.85(\mathrm{~m}$, $2 \mathrm{H}), 2.85-3.5(\mathrm{~m}, 4 \mathrm{H}), 4.13(\mathrm{~d}, J=16.0,1 \mathrm{H}), 4.29(\mathrm{~d}, J=11.6,1 \mathrm{H}), 4.40(\mathrm{~d}, J=16.0,1 \mathrm{H}), 4.65(\mathrm{~d}, J=$ $11.8,1 \mathrm{H}), 5.36(\mathrm{~s}, 2 \mathrm{H}), 7.05-7.45(\mathrm{~m}, 11 \mathrm{H}), 7.58(\mathrm{~d}, J=7.4,2 \mathrm{H}), 7.73(\mathrm{~d}, J=10.0,1 \mathrm{H}), 7.78(\mathrm{~d}, J=8.8$, 1H), 10.76 (s, 1H); HRMS (EI) Calcd for $\mathrm{C}_{39} \mathrm{H}_{42} \mathrm{~N}_{2} \mathrm{O}_{6}$ : 634.3043. Found: 634.3070; Anal. Calcd for $\mathrm{C}_{39} \mathrm{H}_{42} \mathrm{~N}_{2} \mathrm{O}_{6}: \mathrm{C}, 73.79 ; \mathrm{H}, 6.67 ; \mathrm{N}, 4.41$. Found: $\mathrm{C}, 73.43 ; \mathrm{H}, 6.82 ; \mathrm{N}, 4.38$. 


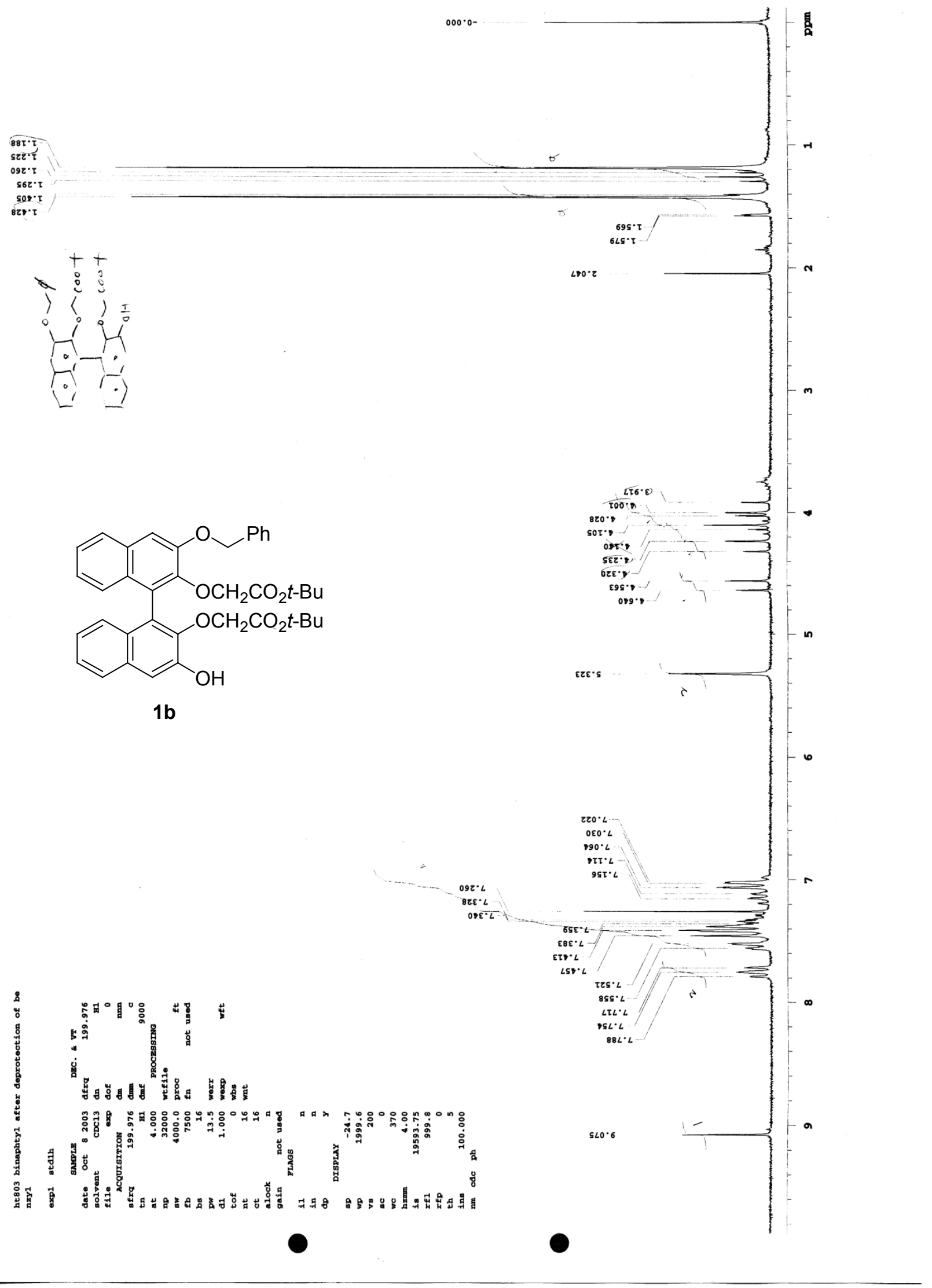




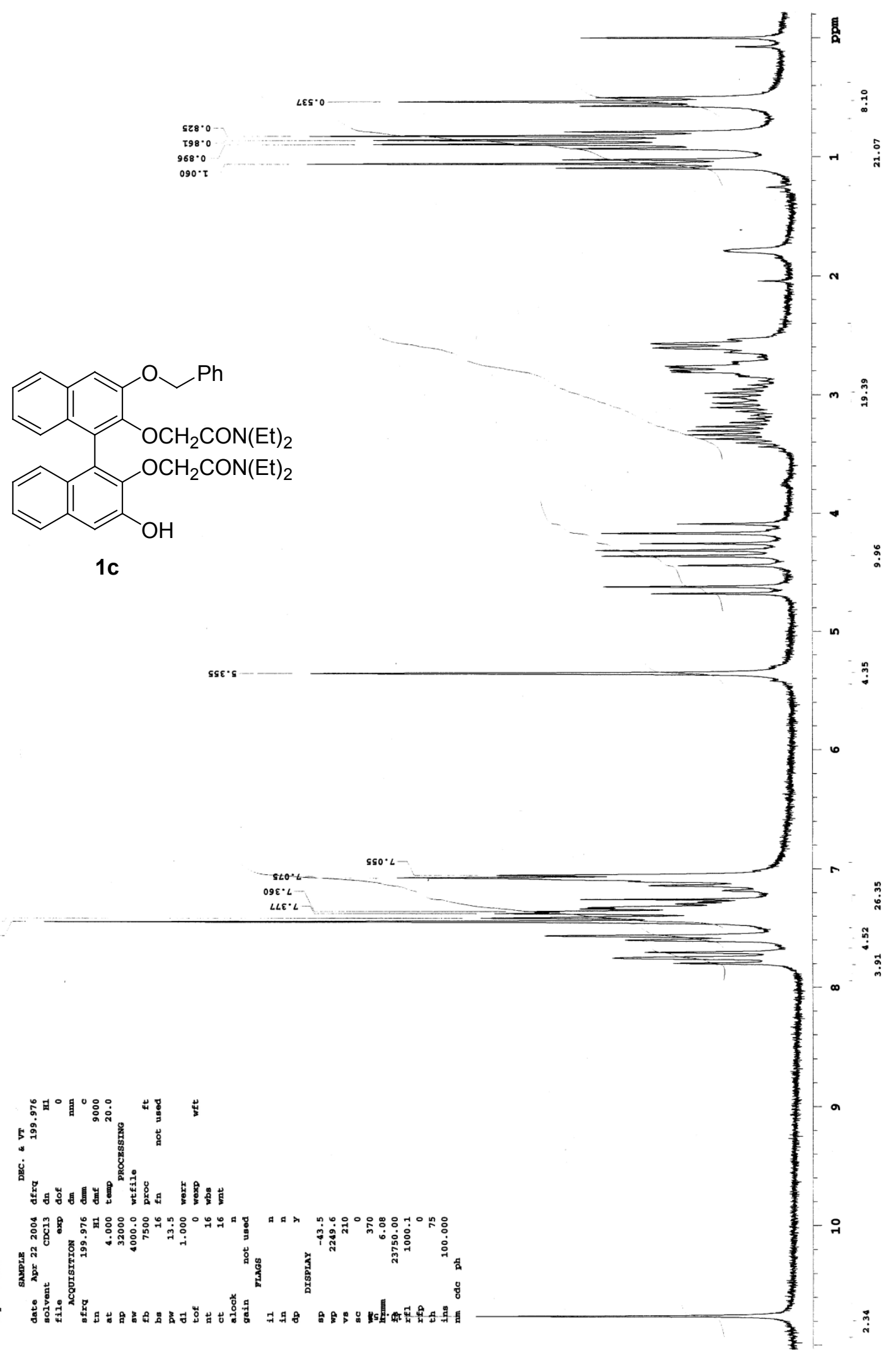


(S)-1d: A mixture of $(S)-33 d(37.0 \mathrm{~g}, 61 \mathrm{mmol})$, ammonium formate (4.6 g, $73 \mathrm{mmol}, 1.2$ eq.) and $10 \%$ palladium on carbon $(3 \mathrm{~g})$ in ethanol $(75 \mathrm{ml})$ and dioxane $(150 \mathrm{ml})$ was stirred for 1.5 hours at $80{ }^{\circ} \mathrm{C}$. After palladium on carbon was filtered off, the filtrate was evaporated. The residue was purified by column chromatography $\left(\mathrm{SiO}_{2}\right.$, hexane/ethyl acetate $\left.=4 / 1\right)$ to afford $(S)-1 \mathbf{d}(16.4 \mathrm{~g}, 52 \%)$ as pale red foam.

$(S)-1 d:[\alpha]_{\mathrm{D}}{ }^{19}=+49.9\left(\mathrm{c}=1.14, \mathrm{CHCl}_{3}\right) ; \mathrm{IR}\left(\mathrm{CHCl}_{3}\right) 3512,2960,1440,1378,1244 \mathrm{~cm}^{-1} ;{ }^{1} \mathrm{H}-\mathrm{NMR}$ $\left(200 \mathrm{MHz}, \mathrm{CDCl}_{3}\right) \delta 0.44(\mathrm{t}, J=7.4,3 \mathrm{H}), 0.57(\mathrm{t}, J=7.2,3 \mathrm{H}), 0.6-1.4(\mathrm{~m}, 8 \mathrm{H}), 3.4-3.7(\mathrm{~m}, 3 \mathrm{H}), 3.95-$ $4.05(\mathrm{~m}, 1 \mathrm{H}), 5.30(\mathrm{~s}, 2 \mathrm{H}), 6.20(\mathrm{~s}, 1 \mathrm{H}), 7.05-7.20(\mathrm{~m}, 4 \mathrm{H}), 7.3-7.5(\mathrm{~m}, 7 \mathrm{H}), 7.5-7.6(\mathrm{~m}, 2 \mathrm{H})$, 7.7-7.85 (m, 2H); HRMS (EI) Calcd for $\mathrm{C}_{35} \mathrm{H}_{36} \mathrm{O}_{4}:$ 520.2614. Found: 520.2640; Anal. Calcd for $\mathrm{C}_{35} \mathrm{H}_{36} \mathrm{O}_{4}: \mathrm{C}_{\text {, }}$ 80.74; H, 6.97. Found: C, 80.37; H, 6.89. 


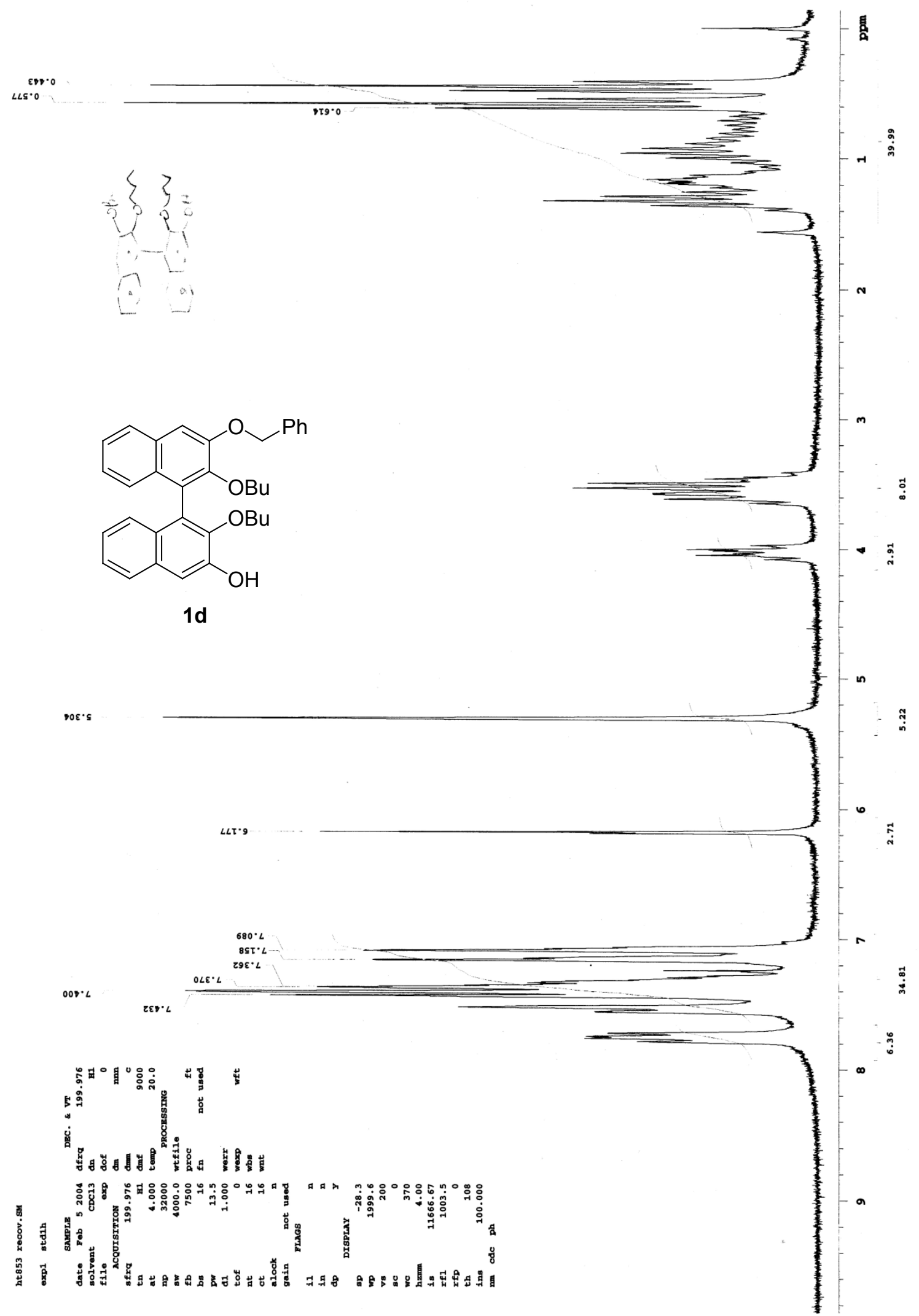


General Procedure for the Synthesis of Compounds 2b-d: The synthesis of $(R, R, R)$ - and $(R, S, R)$ 2b is typical. To a mixture of $\mathrm{CuCl}_{2}(540 \mathrm{mg}, 4.0 \mathrm{mmol})$ in methanol $(10 \mathrm{ml}),(d l)$ - $\alpha$-phenylethylamine (0.64 ml, $5.0 \mathrm{mmol})$ was added under argon atmosphere under ice-bath cooling. After $30 \mathrm{~min}$, a solution of $(R)-1 \mathbf{b}(1.27 \mathrm{~g}, 2.0 \mathrm{mmol})$ in dichloromethane $(10 \mathrm{ml})$ was added and the reaction mixture was stirred for 2 days at $0{ }^{\circ} \mathrm{C}$. The reaction mixture was poured into the mixed solvent of $1 \mathrm{M}$ hydrochloric acid solution and ethyl acetate. The organic layer was washed with water (three times) and brine, and dried over sodium sulfate and evaporated to give a residue. The residue was purified by column chromatography $\left(\mathrm{SiO}_{2}\right.$ hexane/chloroform/ethyl acetate $\left.=8 / 3 / 1\right)$ to afford to $(R, R, R)-\mathbf{2 b}(605$ $\mathrm{mg}, 48 \%)$ and $(R, S, R)-\mathbf{2 b}(69 \mathrm{mg}, 5.4 \%)$.

$(R, R, R)-\mathbf{2 b}$ : colorless foam; $[\alpha]_{\mathrm{D}}{ }^{20}=+51.0\left(\mathrm{c}=0.94, \mathrm{CHCl}_{3}\right) ; \mathrm{IR}(\mathrm{KBr}) 3310,2980,1728,1418,1369$, $1234 \mathrm{~cm}^{-1} ;{ }^{1} \mathrm{H}-\mathrm{NMR}\left(200 \mathrm{MHz}, \mathrm{CDCl}_{3}\right) \delta 1.28(\mathrm{~s}, 18 \mathrm{H}), 1.39(\mathrm{~s}, 18 \mathrm{H}), 4.03(\mathrm{~d}, J=16.6,2 \mathrm{H}), 4.25(\mathrm{~d}, J$ $=16.6,2 \mathrm{H}), 4.27(\mathrm{~d}, J=15.8,2 \mathrm{H}), 4.67(\mathrm{~d}, J=15.8,2 \mathrm{H}), 5.37(\mathrm{~s}, 4 \mathrm{H}), 7.0-7.6(\mathrm{~m}, 26 \mathrm{H}), 7.80(\mathrm{~d}, J=$ 8.0, 2H), $8.82(\mathrm{~s}, 2 \mathrm{H})$; HRMS (FAB) Calcd for $\mathrm{C}_{78} \mathrm{H}_{78} \mathrm{O}_{16}: 1270.5290$. Found: 1270.5297; Anal. Calcd for $\mathrm{C}_{78} \mathrm{H}_{78} \mathrm{O}_{16} \cdot 1 \mathrm{H}_{2} \mathrm{O}: \mathrm{C}, 72.65 ; \mathrm{H}, 6.25$. Found: $\mathrm{C}, 72.58 ; \mathrm{H}, 6.04$.

$(R, S, R)-\mathbf{2 b}$ : colorless foam; $[\alpha]_{\mathrm{D}}{ }^{20}=+12.8\left(\mathrm{c}=1.00, \mathrm{CHCl}_{3}\right)$; IR $(\mathrm{KBr}) 3454,2979,1750,1726,1439$, 1369, $1234 \mathrm{~cm}^{-1} ;{ }^{1} \mathrm{H}-\mathrm{NMR}\left(200 \mathrm{MHz}, \mathrm{CDCl}_{3}\right) \delta 1.28(\mathrm{~s}, 18 \mathrm{H}), 1.38(\mathrm{~s}, 18 \mathrm{H}), 4.21(\mathrm{~d}, J=16.6,2 \mathrm{H})$, $4.23(\mathrm{~d}, J=15.2,2 \mathrm{H}), 4.49(\mathrm{~d}, J=16.6,2 \mathrm{H}), 4.70(\mathrm{~d}, J=15.2,2 \mathrm{H}), 5.37(\mathrm{~s}, 4 \mathrm{H}), 7.0-7.6(\mathrm{~m}, 26 \mathrm{H}), 7.80$ $(\mathrm{d}, J=8.2,2 \mathrm{H}), 8.71(\mathrm{~s}, 2 \mathrm{H})$; HRMS (FAB) Calcd for $\mathrm{C}_{78} \mathrm{H}_{78} \mathrm{O}_{16}$ : 1270.5290. Found: 1270.5302; Anal. Calcd for $\mathrm{C}_{78} \mathrm{H}_{78} \mathrm{O}_{16} \cdot 0.5 \mathrm{H}_{2} \mathrm{O}: \mathrm{C}, 73.16 ; \mathrm{H}, 6.22$. Found: C, 73.24; H, 6.29.

$(S, S, S)-2 \mathrm{c}$ : colorless foam; $[\alpha]_{\mathrm{D}}{ }^{20}=-44.3\left(\mathrm{c}=0.91, \mathrm{CHCl}_{3}\right)$; IR $(\mathrm{KBr}) 3482,2970,1638,1438,1242$, $1118 \mathrm{~cm}^{-1} ;{ }^{1} \mathrm{H}-\mathrm{NMR}\left(200 \mathrm{MHz}, \mathrm{CDCl}_{3}\right) \delta 0.68(\mathrm{t}, J=6.8,6 \mathrm{H}), 0.81(\mathrm{t}, J=7.2,6 \mathrm{H}), 0.9-1.1(\mathrm{~m}, 12 \mathrm{H})$, 2.65-3.00 (m, 8H), 3.05-3.45 (m, 8H), $4.16(\mathrm{~d}, J=15.6,2 \mathrm{H}), 4.34(\mathrm{~d}, J=15.8,2 \mathrm{H}), 4.49(\mathrm{~d}, J=11.8$, $2 \mathrm{H}), 4.77(\mathrm{~d}, J=11.8,2 \mathrm{H}), 5.40(\mathrm{~s}, 4 \mathrm{H}), 7.0-7.35(\mathrm{~m}, 22 \mathrm{H}), 7.63(\mathrm{~d}, J=7.0,4 \mathrm{H}), 7.81(\mathrm{~d}, J=8.2,2 \mathrm{H})$, 
10.55 (s, 2H); HRMS (FAB) Calcd for $\mathrm{C}_{78} \mathrm{H}_{83} \mathrm{~N}_{4} \mathrm{O}_{12}\left(\mathrm{M}+\mathrm{H}^{+}\right)$: 1267.6007. Found: 1267.6042; Anal. Calcd for $\mathrm{C}_{78} \mathrm{H}_{82} \mathrm{~N}_{4} \mathrm{O}_{12} \cdot 1 \mathrm{H}_{2} \mathrm{O}$ : C, 72.88; H, 6.59; N, 4.36. Found: C, 73.19; H, 6.63; N, 4.29.

$(S, R, S)-2 \mathrm{c}:$ colorless foam; $[\alpha]_{\mathrm{D}}{ }^{20}=-17.7\left(\mathrm{c}=0.87, \mathrm{CHCl}_{3}\right)$; IR $(\mathrm{KBr}) 3481,3062,2972,1638,1439$, 1294, $1118 \mathrm{~cm}^{-1} ;{ }^{1} \mathrm{H}-\mathrm{NMR}\left(200 \mathrm{MHz}, \mathrm{CDCl}_{3}\right) \delta 0.60(\mathrm{t}, J=6.8,6 \mathrm{H}), 0.8-1.1(\mathrm{~m}, 18 \mathrm{H}), 2.5-3.5(\mathrm{~m}$, $16 \mathrm{H}), 4.29(\mathrm{~d}, J=15.4,2 \mathrm{H}), 4.45(\mathrm{~d}, J=11.2,2 \mathrm{H}), 4.48(\mathrm{~d}, J=15.8,2 \mathrm{H}), 4.80(\mathrm{~d}, J=11.2,2 \mathrm{H}), 5.42(\mathrm{~s}$, 4H), 7.05-7.55 (m, 22H), $7.65(\mathrm{~d}, J=6.8,4 \mathrm{H}), 7.82(\mathrm{~d}, J=8.2,2 \mathrm{H}), 10.51(\mathrm{~s}, 2 \mathrm{H})$; HRMS (FAB) Calcd for $\mathrm{C}_{78} \mathrm{H}_{83} \mathrm{~N}_{4} \mathrm{O}_{12}\left(\mathrm{M}+\mathrm{H}^{+}\right)$: 1267.6007. Found: 1267.6002; Anal. Calcd for $\mathrm{C}_{78} \mathrm{H}_{82} \mathrm{~N}_{4} \mathrm{O}_{12} \cdot 1 \mathrm{H}_{2} \mathrm{O}$ : C, 72.88; H, 6.59; N, 4.36. Found: C, 72.58; H, 6.45; N, 4.31.

$(S, S, S)-2 d:\left(\right.$ colorless needle); $\mathrm{Mp} 81-83{ }^{\circ} \mathrm{C} ;[\alpha]_{\mathrm{D}}{ }^{20}=-36.8\left(\mathrm{c}=1.01, \mathrm{CHCl}_{3}\right) ; \mathrm{IR}(\mathrm{KBr}) 3513,3064$, 2957, 2870, 1594, 1439, $1376 \mathrm{~cm}^{-1} ;{ }^{1} \mathrm{H}-\mathrm{NMR}\left(200 \mathrm{MHz}, \mathrm{CDCl}_{3}\right) \delta 0.58(\mathrm{t}, J=7.3,12 \mathrm{H}), 0.70-1.20(\mathrm{~m}$, $8 \mathrm{H}), 1.25-1.40(\mathrm{~m}, 8 \mathrm{H}), 3.5-3.9(\mathrm{~m}, 6 \mathrm{H}), 4.1-4.3(\mathrm{~m}, 2 \mathrm{H}), 5.36(\mathrm{~s}, 4 \mathrm{H}), 6.31(\mathrm{~s}, 2 \mathrm{H}), 7.0-7.5(\mathrm{~m}, 22 \mathrm{H})$, $7.57(\mathrm{~d}, J=7.6,4 \mathrm{H}), 7.81(\mathrm{~d}, J=7.6,2 \mathrm{H})$; HRMS Calcd for $\mathrm{C}_{70} \mathrm{H}_{70} \mathrm{O}_{8}: 1038.5071$. Found: 1038.5055; Anal. Calcd for $\mathrm{C}_{70} \mathrm{H}_{70} \mathrm{O}_{8}:$ C, 80.90; H, 6.79. Found: C, 80.89; H, 6.79.

$(S, R, S)-2 d$ : (colorless foam); $[\alpha]_{\mathrm{D}}{ }^{20}=+8.5\left(\mathrm{c}=0.81, \mathrm{CHCl}_{3}\right) ; \mathrm{IR}(\mathrm{KBr}) 3513,3063,2957,2871,1595$, 1440, $1377 \mathrm{~cm}^{-1} ;{ }^{1} \mathrm{H}-\mathrm{NMR}\left(200 \mathrm{MHz}, \mathrm{CDCl}_{3}\right) \delta$ 0.5-0.6 (m, 12H), 0.7-1.1 (m, 8H), 1.2-1.4 (m, 8H), 3.6-3.8 (m, 6H), 4.1-4.2 (m, 2H), $5.36(\mathrm{~s}, 4 \mathrm{H}), 6.03(\mathrm{~s}, 2 \mathrm{H}), 7.1-7.5(\mathrm{~m}, 22 \mathrm{H}), 7.57(\mathrm{~d}, J=6.6,4 \mathrm{H}), 7.82$ (d, $J=8.0,2 \mathrm{H}$ ); HRMS Calcd for $\mathrm{C}_{70} \mathrm{H}_{70} \mathrm{O}_{8}$ : 1038.5071. Found: 1038.5066; Anal. Calcd for $\mathrm{C}_{70} \mathrm{H}_{70} \mathrm{O}_{8}$ : C, 80.90; H, 6.79. Found: C, 80.71; H, 6.87. 


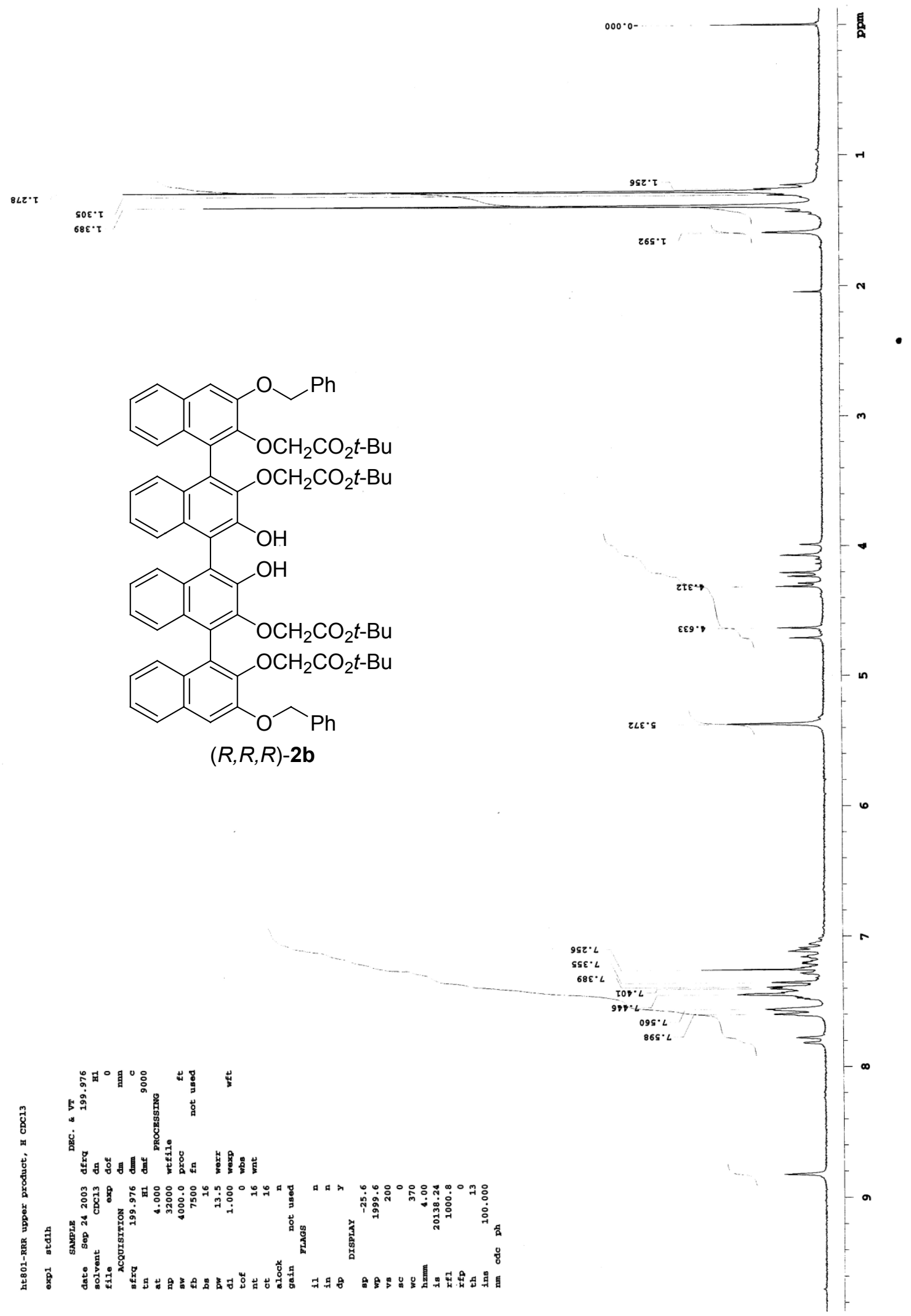




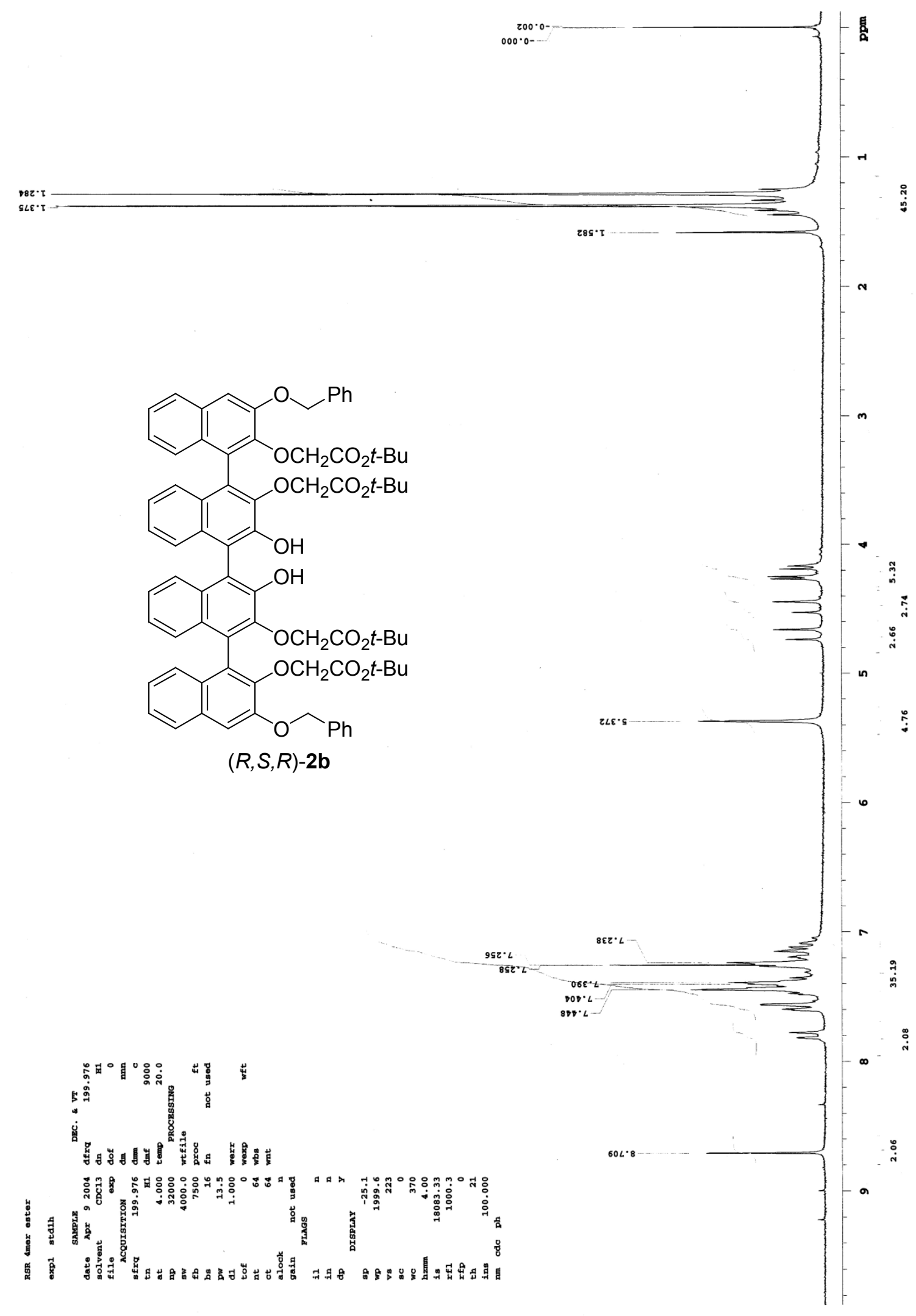




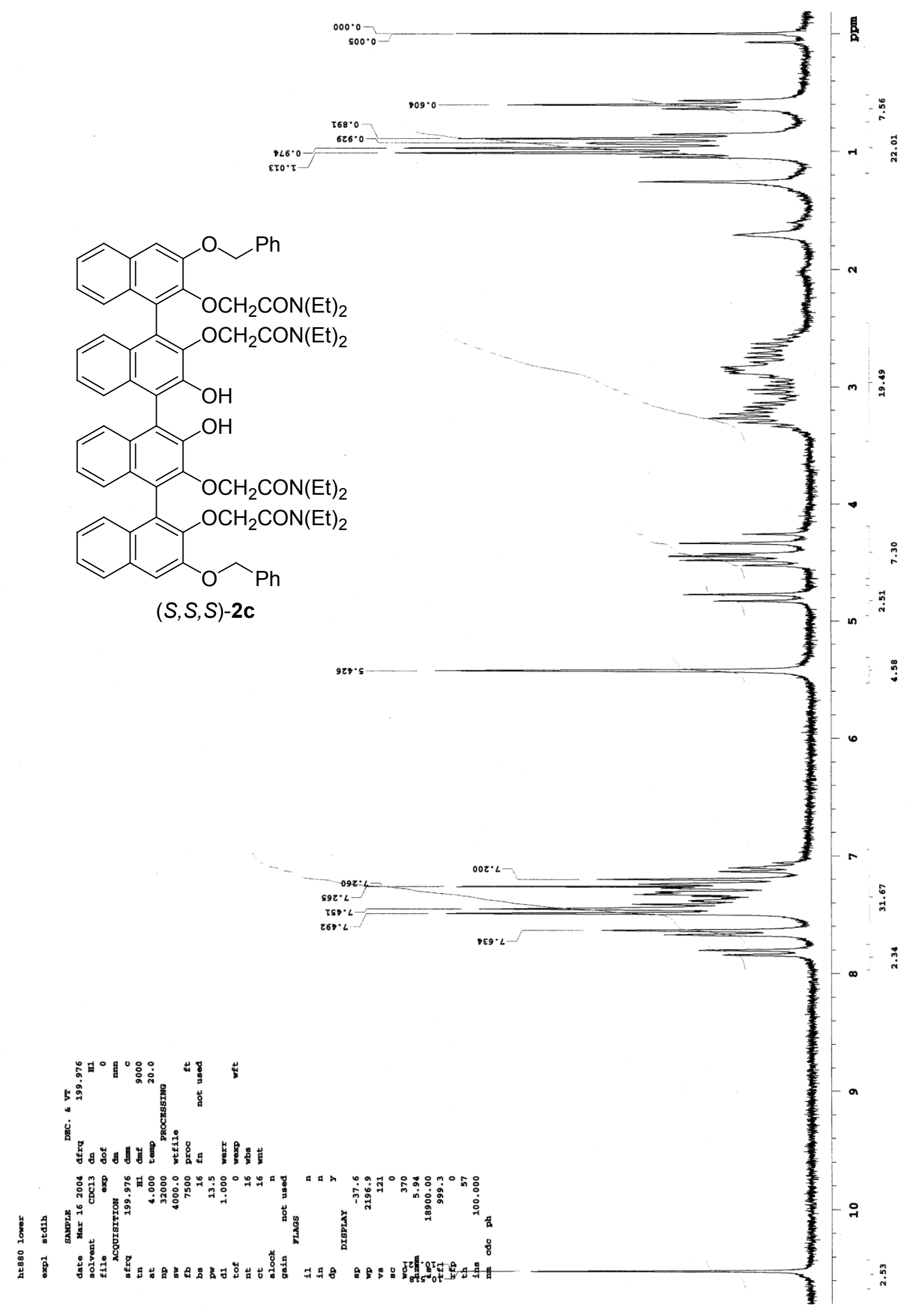




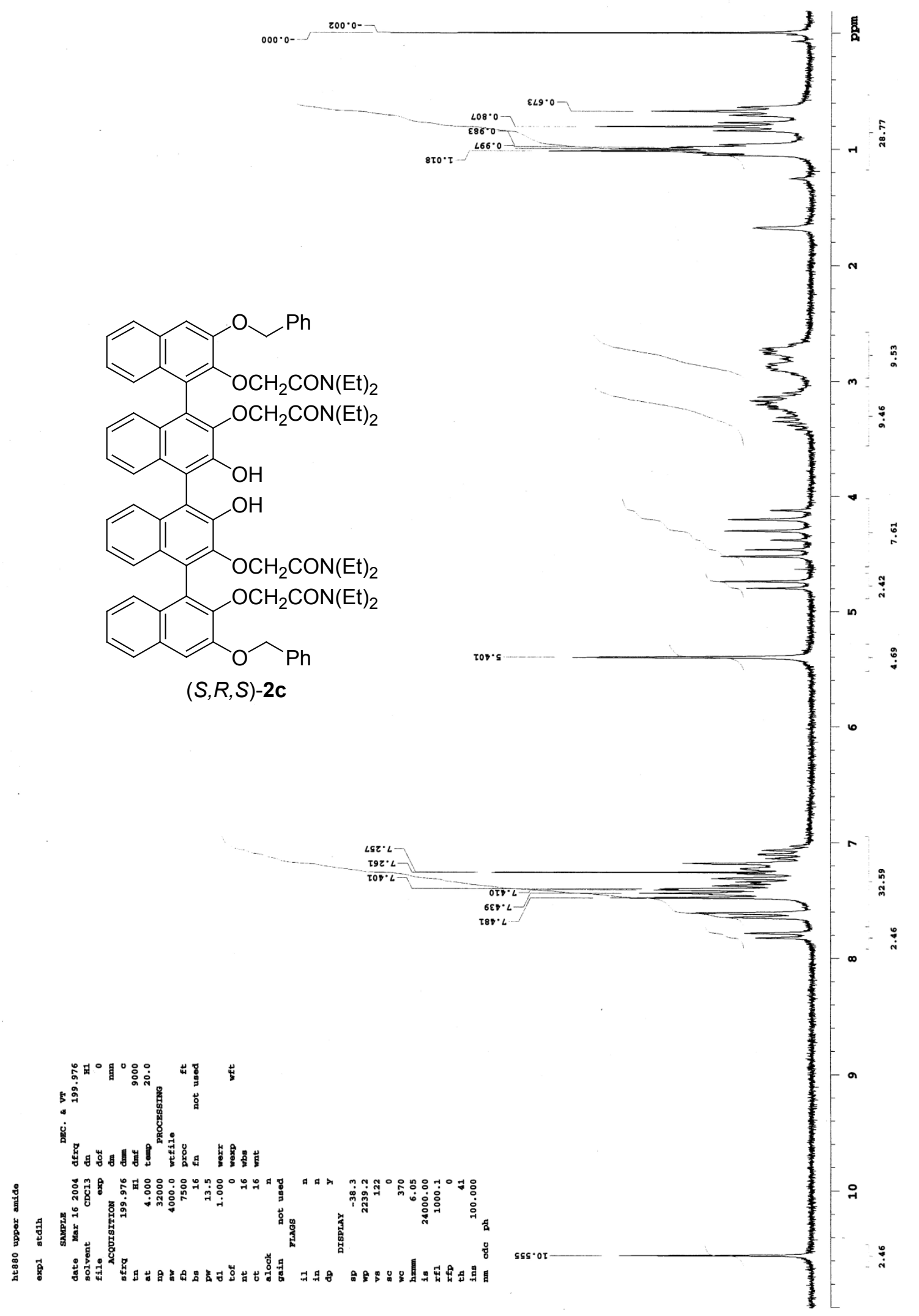



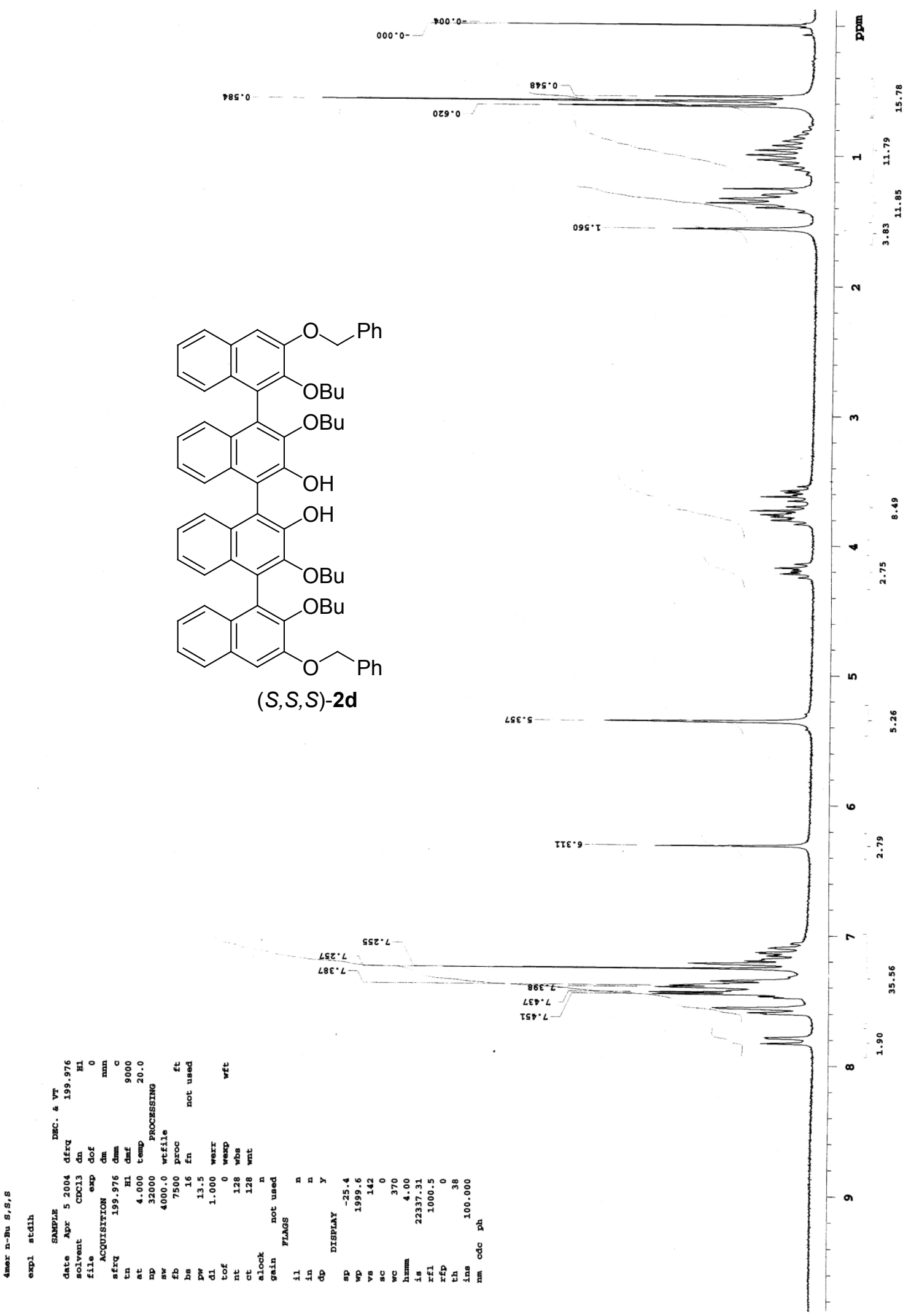


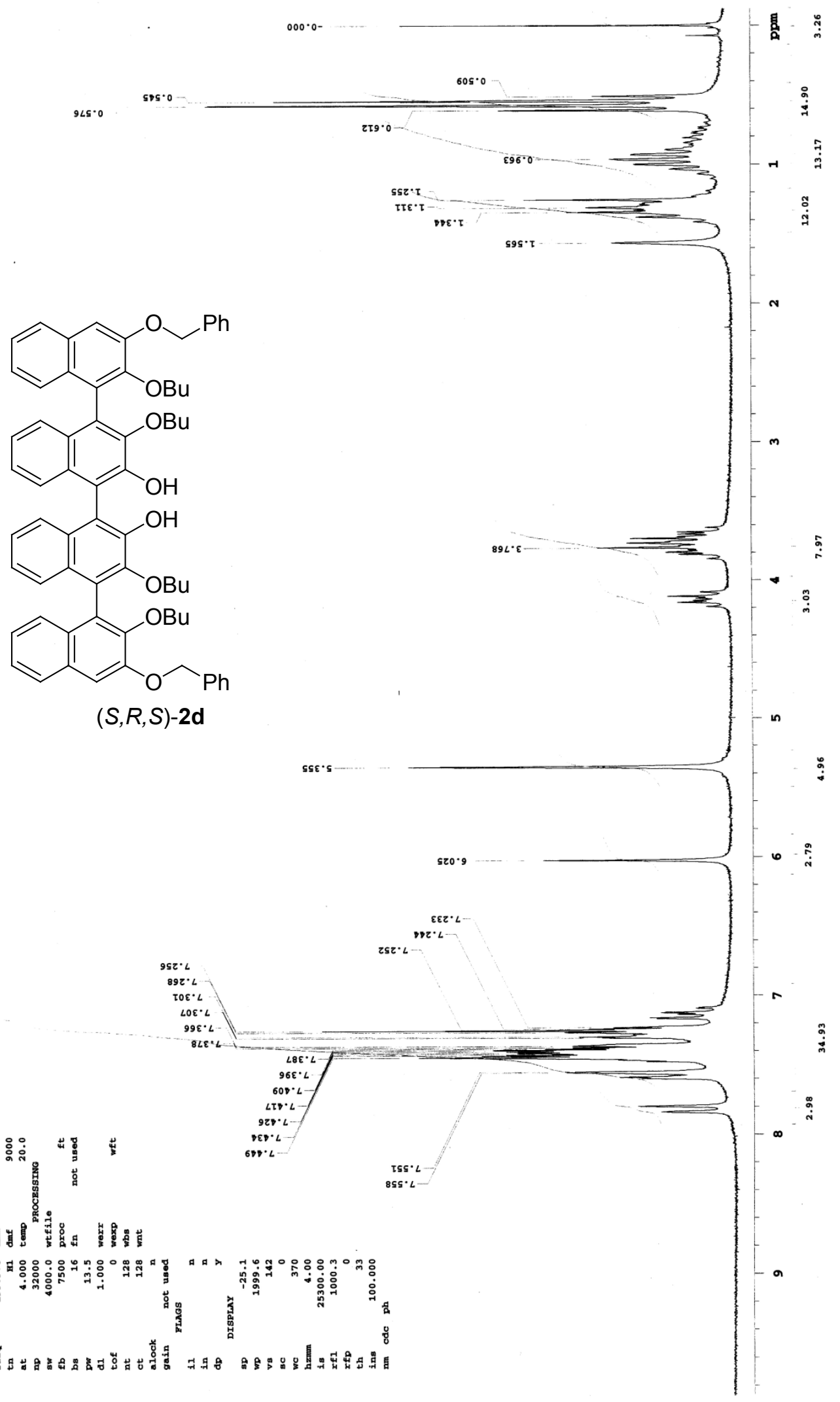

$P$ 
$(R, R, R)-\mathbf{3 4 b}$ : To a solution of $(R, R, R)-\mathbf{2 b}(714 \mathrm{mg}, 0.56 \mathrm{mmol})$ and $N, N$-diisopropylethylamine $(0.29$ $\mathrm{ml}, 1.68 \mathrm{mmol})$ in acetonitrile $(12 \mathrm{ml})$ and methanol $(3 \mathrm{ml})$, trimethyldiazomethane $(2.0 \mathrm{M}$ solution in diethyl ether, $1.4 \mathrm{ml}$ ) was added dropwise at room temperature and stirred for 3 hours. The reaction mixture was poured into the mixed solvent of $1 \mathrm{M}$ hydrochloric acid solution and ethyl acetate. The organic layer was washed with water (three times) and brine, dried over sodium sulfate and evaporated to give a residue. The residue was purified by column chromatography $\left(\mathrm{SiO}_{2}\right.$ hexane/ethyl acetate $=$ $4 / 1)$ to afford to $(R, R, R)-34 \mathbf{b}(470 \mathrm{mg}, 64 \%)$ as colorless foam.

$[\alpha]_{\mathrm{D}}^{20}=+74.2\left(\mathrm{c}=1.02, \mathrm{CHCl}_{3}\right) ; \mathrm{IR}(\mathrm{KBr}) 2929,1757,1458,1397,1228,1154 \mathrm{~cm}^{-1} ;{ }^{1} \mathrm{H}-\mathrm{NMR}(200$ $\left.\mathrm{MHz}, \mathrm{CDCl}_{3}\right) \delta 1.29(\mathrm{~s}, 36 \mathrm{H}), 3.76(\mathrm{~s}, 6 \mathrm{H}), 4.25(\mathrm{~d}, J=15.2,2 \mathrm{H}), 4.45(\mathrm{~d}, J=15.4,2 \mathrm{H}), 4.65(\mathrm{~d}, J=$ 15.4, 2H), $4.72(\mathrm{~d}, J=15.2,2 \mathrm{H}), 5.37(\mathrm{~s}, 4 \mathrm{H}), 7.1-7.6(\mathrm{~m}, 26 \mathrm{H}), 7.79(\mathrm{~d}, J=8.2,2 \mathrm{H})$; HRMS $\left(\mathrm{FAB}^{+}\right)$ Calcd for $\mathrm{C}_{80} \mathrm{H}_{82} \mathrm{O}_{16}$ : 1298.5603. Found: 1298.5641; Anal. Calcd for $\mathrm{C}_{80} \mathrm{H}_{82} \mathrm{O}_{16} \cdot 0.5 \mathrm{H}_{2} \mathrm{O}: \mathrm{C}, 73.43$; $\mathrm{H}$, 6.39. Found: C, 73.39; H, 6.61 . 


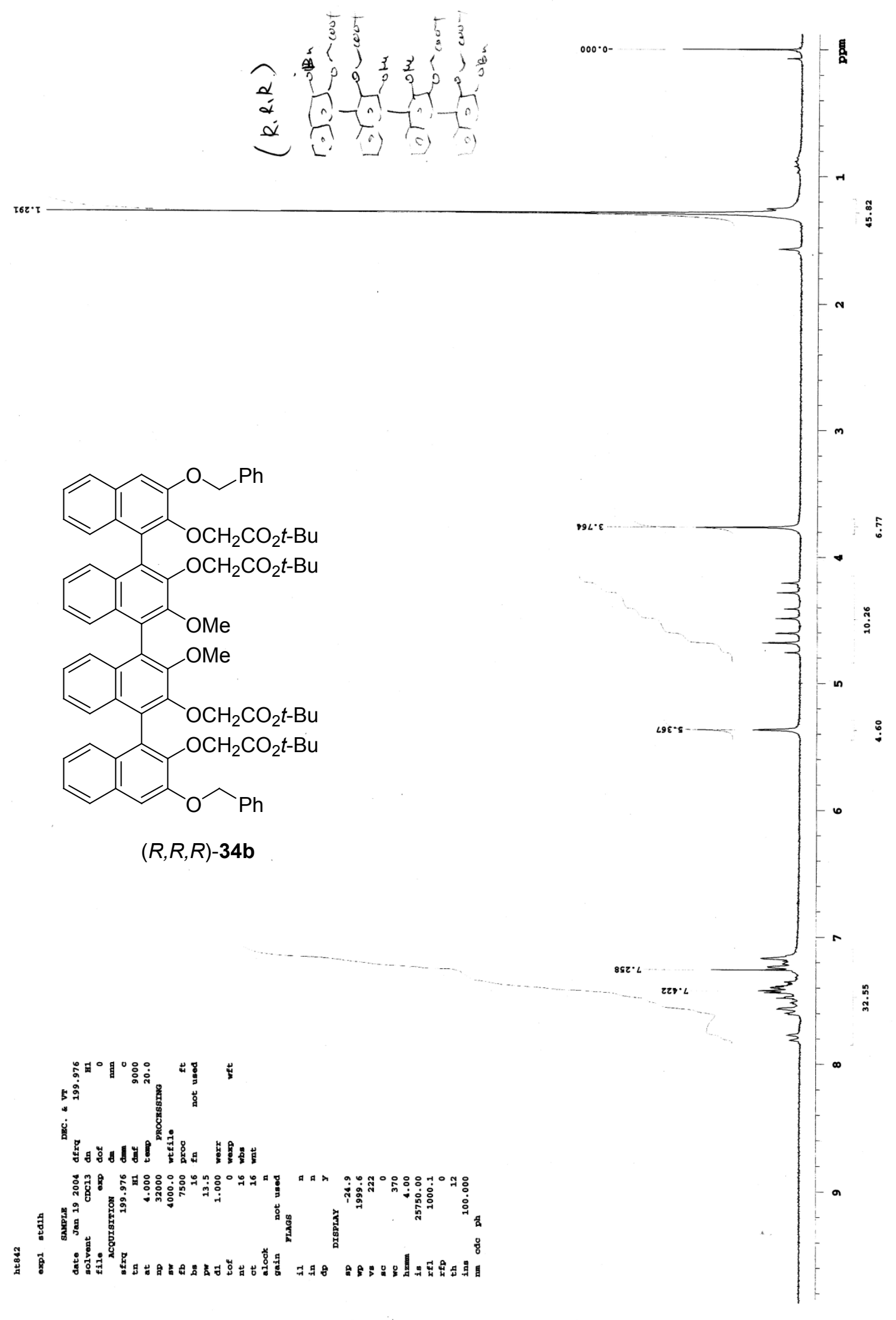


$(S, S, S)-17$ : To a suspension of $(S, S, S)-2 \mathrm{c}(3.29 \mathrm{~g}, 2.6 \mathrm{mmol})$ and potassium carbonate $(1.08 \mathrm{~g}, 7.8$ mmol, 3.0 eq.) in DMF $(8 \mathrm{ml})$, iodomethane $(0.81 \mathrm{ml}, 13 \mathrm{mmol}, 5.0$ eq. $)$ was added. The reaction mixture was stirred for 19 hours at room temperature. The reaction mixture was poured into the mixed solvent of ethyl acetate and water. The organic layer was separated, washed successively with $0.1 \mathrm{M}$ hydrochloric acid solution, water (twice) and brine. After dried over sodium sulfate, the solvent was evaporated in vacuo to afford pale yellow powder $(3.4 \mathrm{~g}, 100 \%)$. Crude $(S, S, S)-17$ was directly used for the next step without further purification. A small part of the sample was subjected to further purification by PTLC to give an analytical sample. yellow powder; $\mathrm{Mp}, 125^{\circ} \mathrm{C} ;[\alpha]_{\mathrm{D}}{ }^{20}=-68.8(\mathrm{c}=$ 1.28, $\left.\mathrm{CHCl}_{3}\right) ; \mathrm{IR}\left(\mathrm{CHCl}_{3}\right) 3445,3006,2936,1640,1459,1442 \mathrm{~cm}^{-1} ;{ }^{1} \mathrm{H}-\mathrm{NMR}\left(200 \mathrm{MHz}, \mathrm{CDCl}_{3}\right) \delta$ $0.58(\mathrm{t}, J=7.1,6 \mathrm{H}), 0.74(\mathrm{t}, J=6.9,6 \mathrm{H}), 0.9-1.05(\mathrm{~m}, 12 \mathrm{H}), 2.66(\mathrm{q}, J=7.4,4 \mathrm{H}), 2.9-3.3(\mathrm{~m}, 12 \mathrm{H})$, $3.80(\mathrm{~s}, 2 \mathrm{H}), 4.31(\mathrm{~d}, J=11.8,2 \mathrm{H}), 4.55(\mathrm{~d}, J=12.2,2 \mathrm{H}), 4.73(\mathrm{~d}, J=13,2 \mathrm{H}), 4.80(\mathrm{~d}, J=12.4,2 \mathrm{H})$, $5.36(\mathrm{~s}, 4 \mathrm{H}), 7.1-7.5(\mathrm{~m}, 22 \mathrm{H}), 7.5-7.6(\mathrm{~m}, 4 \mathrm{H}), 7.81(\mathrm{~d}, J=8.2,2 \mathrm{H})$; HRMS (FAB $\left.{ }^{+}\right)$Calcd for $\mathrm{C}_{80} \mathrm{H}_{87} \mathrm{~N}_{4} \mathrm{O}_{12}$ : 1295.6321. Found: 1295.6315; Anal. Calcd for $\mathrm{C}_{80} \mathrm{H}_{86} \mathrm{~N}_{4} \mathrm{O}_{12} \cdot 1.5 \mathrm{H}_{2} \mathrm{O}$ : C, 72.65; H, 6.78; N, 4.24. Found: C, 72.58; H, 6.70; N, 4.30. 


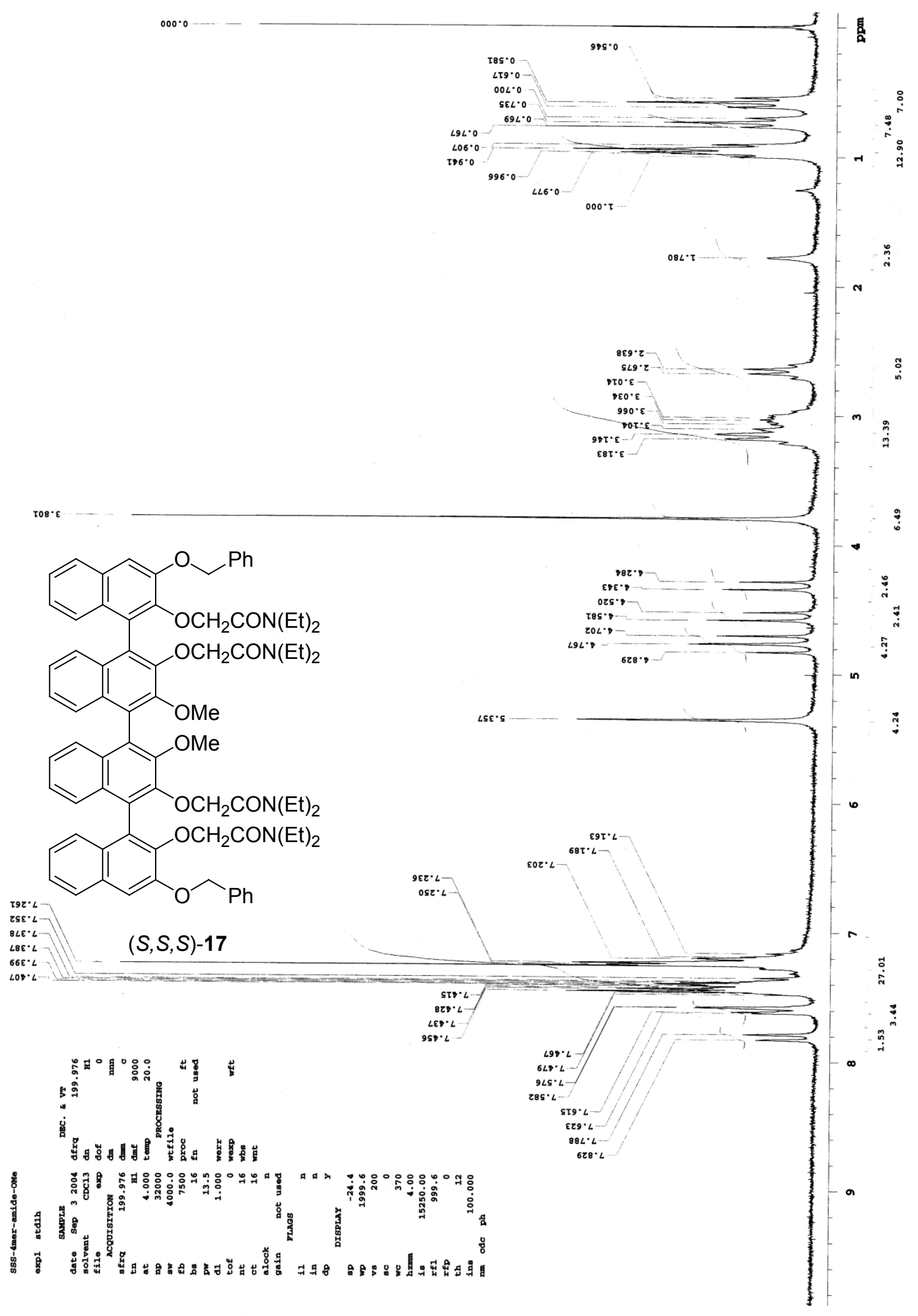


$(S, S, S)-34 \mathbf{d}:(S, S, S)-\mathbf{3 4 d}$ was synthesized similar to $(S, S, S)$-17; $93 \%$ yield; pale yellow gum; $[\alpha]_{\mathrm{D}}{ }^{20}=$ $81.6\left(\mathrm{c}=0.75, \mathrm{CHCl}_{3}\right) ; \mathrm{IR}(\mathrm{KBr}) 2956,1440,1375,1246 \mathrm{~cm}^{-1} ;{ }^{1} \mathrm{H}-\mathrm{NMR}\left(200 \mathrm{MHz}, \mathrm{CDCl}_{3}\right) \delta 0.53(\mathrm{t}, J$ $=7.1,6 \mathrm{H}), 0.65(\mathrm{t}, J=7.2,6 \mathrm{H}), 0.7-1.5(\mathrm{~m}, 16 \mathrm{H}), 3.78(\mathrm{~s}, 6 \mathrm{H}), 3.6-3.9(\mathrm{~m}, 4 \mathrm{H}), 4.0-4.3(\mathrm{~m}, 4 \mathrm{H}), 5.37(\mathrm{~s}$, 4H), 7.10-7.55 (m, 22H), $7.59(\mathrm{~d}, J=7.8,4 \mathrm{H}), 7.81(\mathrm{~d}, J=8.2,2 \mathrm{H})$; HRMS (FAB $\left.{ }^{+}\right)$Calcd for $\mathrm{C}_{72} \mathrm{H}_{74} \mathrm{O}_{8}$ : 1066.5384. Found: 1066.5376; Anal. Calcd for $\mathrm{C}_{72} \mathrm{H}_{74} \mathrm{O}_{8}$ : C, 81.02; H, 6.99. Found: C, 81.06; H, 6.93 . 


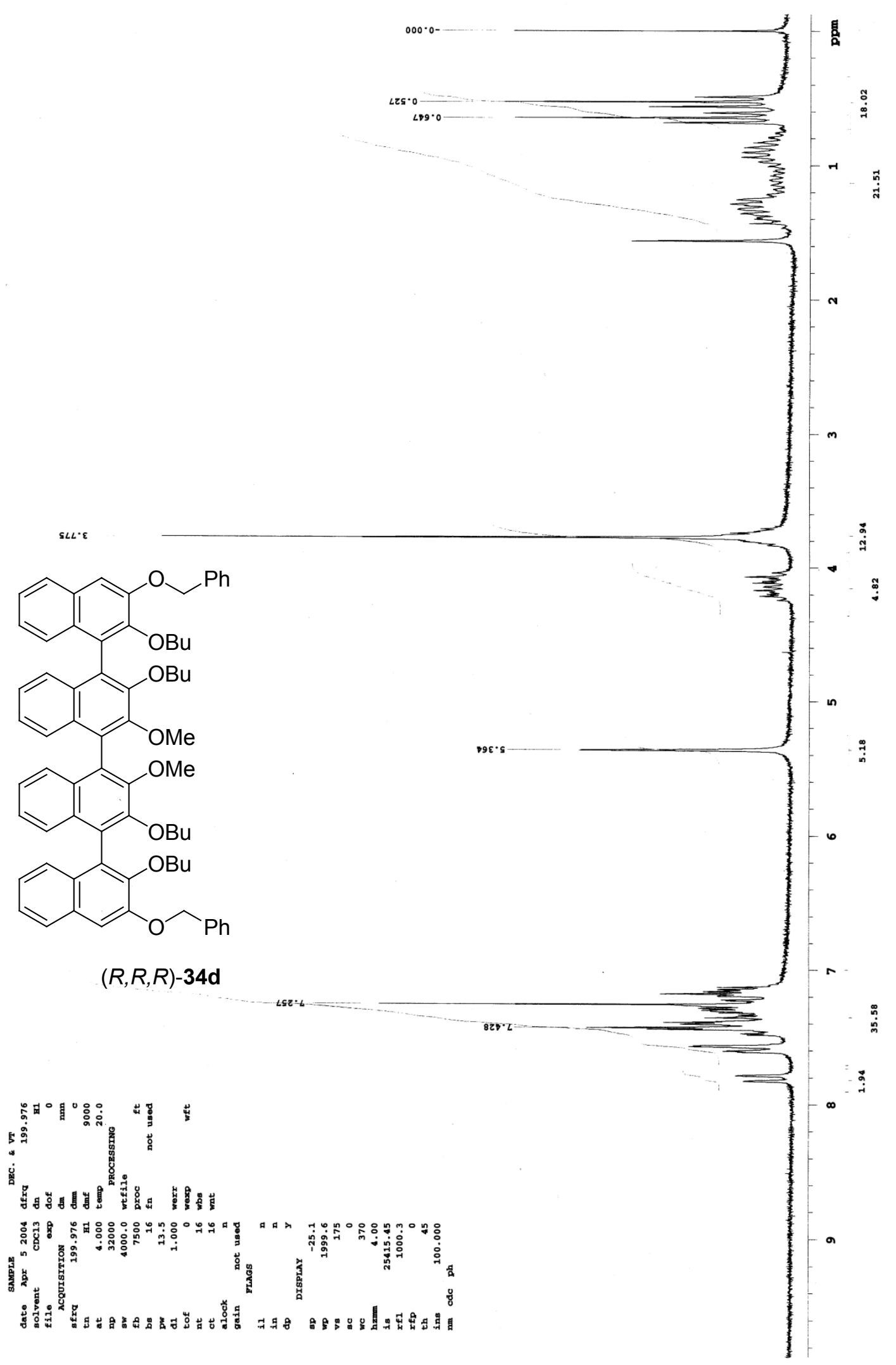


General Procedure for the Synthesis of Compounds 7b-d: The synthesis of $(R, R, R)-7 \mathbf{b}$ is typical. A suspension of $(R, R, R)-\mathbf{3 4 b}(1.05 \mathrm{~g}, 0.81 \mathrm{mmol})$ and $10 \%$ palladium on carbon $(85 \mathrm{mg})$ in THF (15 $\mathrm{ml}$ ), was stirred under hydrogen atmosphere for 3 hours at room temperature with monitoring the reaction by TLC (hexane/diethyl ether/chloroform $=10 / 3 / 3$ ). After palladium on carbon was filtered off, the filtrate was evaporated to give a residue. The residue was purified by column chromatography $\left(\mathrm{SiO}_{2}\right.$, hexane/diethyl ether/chloroform $\left.=10 / 3 / 3\right)$ to afford $(R, R, R)-7 \mathbf{b}(422 \mathrm{mg}, 43 \%)$ and recovered $(R, R, R)-34 \mathbf{b}(220 \mathrm{mg})$. (colorless foam); $[\alpha]_{\mathrm{D}}{ }^{20}=+87.2\left(\mathrm{c}=0.87, \mathrm{CHCl}_{3}\right) ; \mathrm{IR}(\mathrm{KBr}) 3324,3015,1749$, 1227, $1205 \mathrm{~cm}^{-1} ;{ }^{1} \mathrm{H}-\mathrm{NMR}\left(200 \mathrm{MHz}, \mathrm{CDCl}_{3}\right) \delta 1.30(\mathrm{~s}, 36 \mathrm{H}), 3.76(\mathrm{~s}, 3 \mathrm{H}), 3.80(\mathrm{~s}, 3 \mathrm{H}), 4.1-4.8(\mathrm{~m}$, 8H), $5.37(\mathrm{~s}, 2 \mathrm{H}), 7.1-7.6(\mathrm{~m}, 21 \mathrm{H}), 7.80(\mathrm{~d}, J=8.2,2 \mathrm{H}), 9.07(\mathrm{~s}, 1 \mathrm{H})$; HRMS (FAB $\left.{ }^{+}\right) \mathrm{Calcd}_{\text {for }}$ $\mathrm{C}_{73} \mathrm{H}_{76} \mathrm{O}_{16}:$ 1208.5133. Found: 1208.5167; Anal. Calcd for $\mathrm{C}_{73} \mathrm{H}_{76} \mathrm{O}_{16} \cdot \mathrm{H}_{2} \mathrm{O}: \mathrm{C}, 71.43 ; \mathrm{H}, 6.41$. Found: C, $71.43 ; \mathrm{H}, 6.36$.

$(S, S, S)-7 \mathrm{c}: 57 \%$ yield; white powder; $\mathrm{Mp}, 105{ }^{\circ} \mathrm{C} ;[\alpha]_{\mathrm{D}}{ }^{21}=-83.6\left(\mathrm{c}=0.60, \mathrm{CHCl}_{3}\right)$; IR $(\mathrm{KBr}) 3641$, 2971, 1641, 1548, 1511, $1442 \mathrm{~cm}^{-1} ;{ }^{1} \mathrm{H}-\mathrm{NMR}\left(200 \mathrm{MHz}, \mathrm{CDCl}_{3}\right) \delta 0.57(\mathrm{t}, J=7.0,3 \mathrm{H}), 0.60(\mathrm{t}, J=7.0$, 2H), $0.78(\mathrm{t}, J=6.8,3 \mathrm{H}), 0.85-1.05(\mathrm{~m}, 12 \mathrm{H}), 1.12(\mathrm{t}, J=7.4,3 \mathrm{H}), 2.50-2.75(\mathrm{~m}, 4 \mathrm{H}), 2.75-3.25(\mathrm{~m}$, $10 \mathrm{H}), 3.25-3.50(\mathrm{~m}, 2 \mathrm{H}), 3.81(\mathrm{~s}, 3 \mathrm{H}), 3.84(\mathrm{~s}, 3 \mathrm{H}), 4.35-4.85(\mathrm{~m}, 8 \mathrm{H}), 5.36(\mathrm{~s}, 2 \mathrm{H}), 7.10-7.55(\mathrm{~m}, 19 \mathrm{H})$, 7.55-7.60 (m, 2H), 7.75-7.85 (m, 2H), $10.81(\mathrm{~s}, 1 \mathrm{H})$; HRMS (FAB) Calcd for $\mathrm{C}_{73} \mathrm{H}_{81} \mathrm{O}_{12} \mathrm{~N}_{4}: 1205.5851$. Found: 1205.5836;

$(S, S, S)-7 \mathbf{d}: 40 \%$ yield; colorless gum; $[\alpha]_{\mathrm{D}}{ }^{18}=-71\left(\mathrm{c}=0.34, \mathrm{CHCl}_{3}\right) ; \mathrm{IR}\left(\mathrm{CHCl}_{3}\right) 3517,2959,1442$, 1377, 1347, $1241 \mathrm{~cm}^{-1} ;{ }^{1} \mathrm{H}-\mathrm{NMR}\left(200 \mathrm{MHz}, \mathrm{CDCl}_{3}\right) \delta$ 0.5-1.6 (m, 28H), 3.6-3.9 (m, 4H), $3.75(\mathrm{~s}, 3 \mathrm{H})$, $3.90(\mathrm{~s}, 3 \mathrm{H}), 4.0-4.3(\mathrm{~m}, 4 \mathrm{H}), 5.36(\mathrm{~s}, 2 \mathrm{H}), 6.20(\mathrm{~s}, 1 \mathrm{H}), 7.1-7.5(\mathrm{~m}, 19 \mathrm{H}), 7.58(\mathrm{~d}, J=7.4,2 \mathrm{H}), 7.80(\mathrm{~d}$, $J=8.4,2 \mathrm{H}$ ); HRMS (FAB) Calcd for $\mathrm{C}_{65} \mathrm{H}_{68} \mathrm{O}_{8}$ : 976.4914. Found: 976.4921; Anal. Calcd for $\mathrm{C}_{65} \mathrm{H}_{68} \mathrm{O}_{8}$ : C, 79.89; H, 7.01. Found: C, 79.73; H, 7.15. 


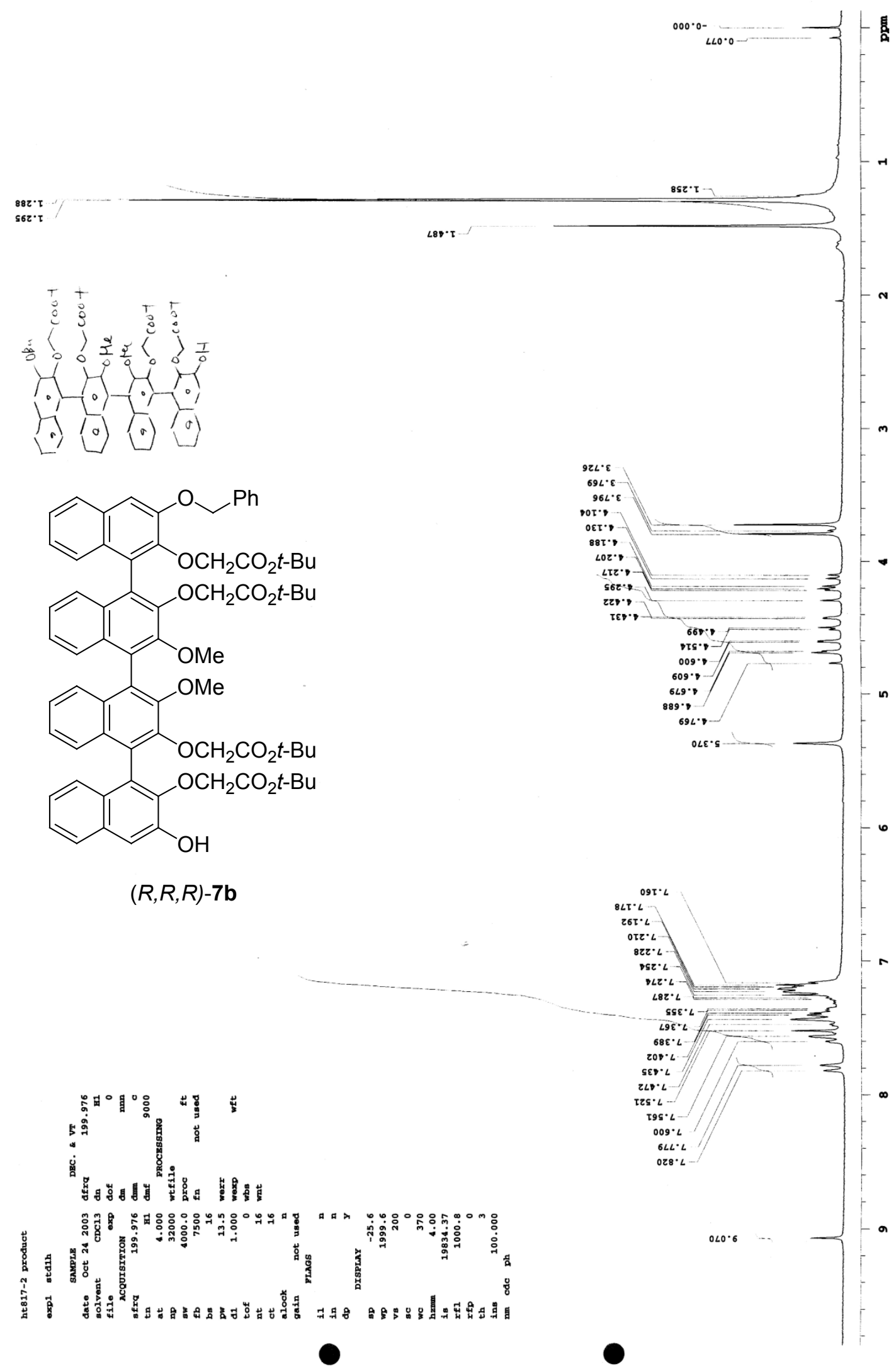




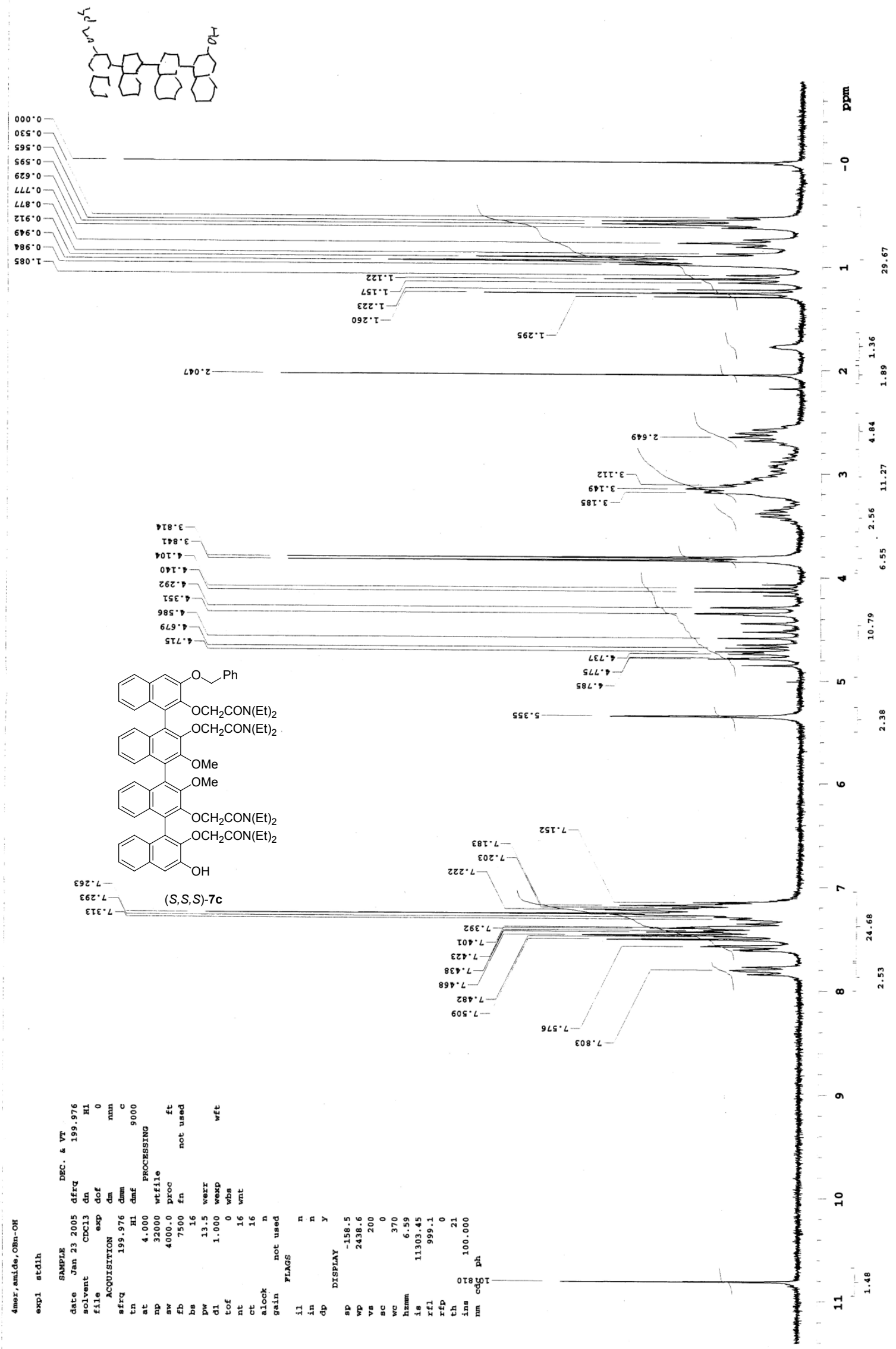




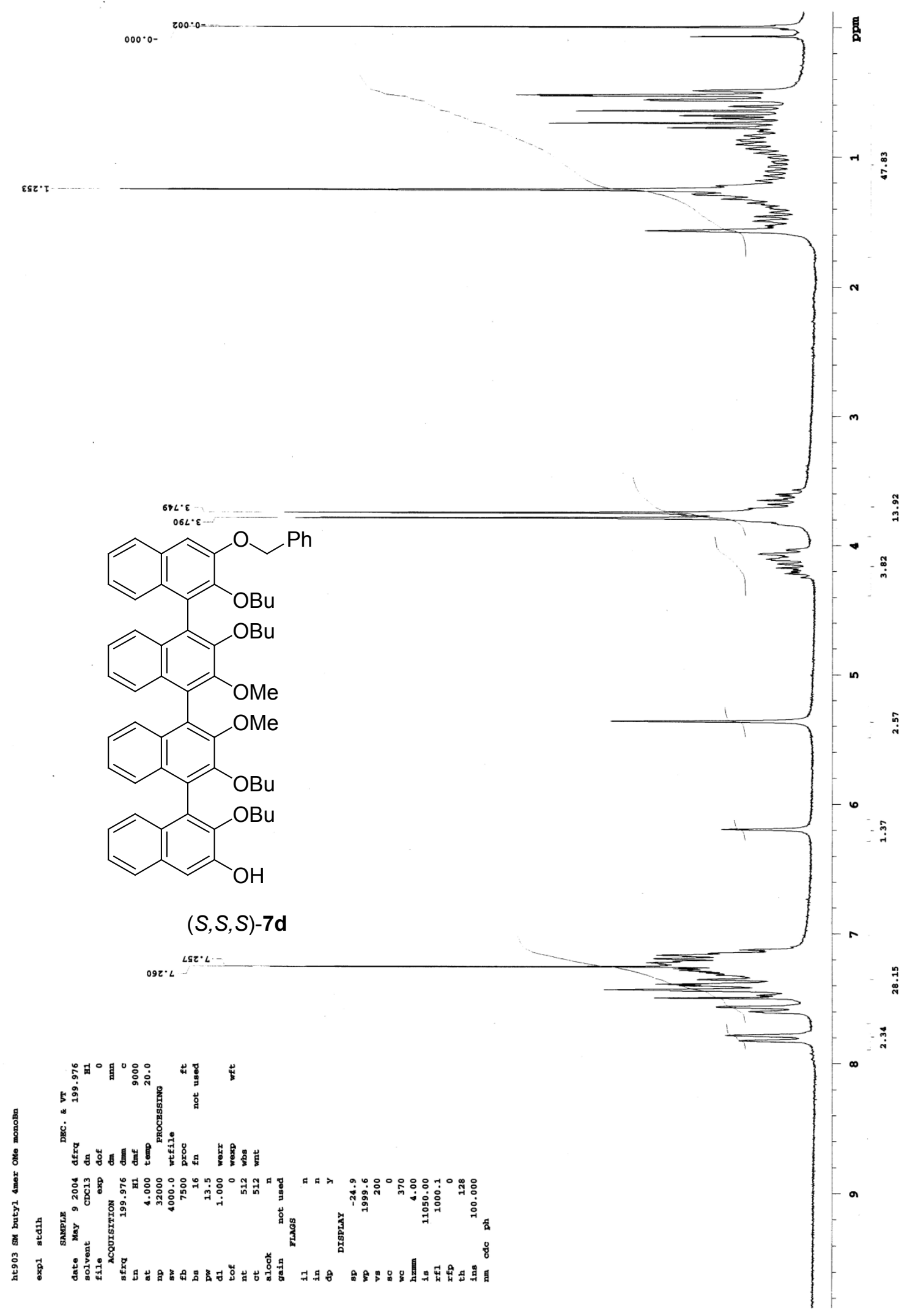


General Procedure for the Synthesis of Compounds 8b-d: The synthesis of $(S, S, S, S, S, S, S)$ - and $(S, S, S, R, S, S, S)-8 \mathbf{d}$ is typical. To a suspension of $\mathrm{CuCl}_{2}(27 \mathrm{mg}, 0.2 \mathrm{mmol})$ in methanol $(1 \mathrm{ml}),(d l)-\alpha-$ phenylethylamine $(32 \mu \mathrm{l}, 0.25 \mathrm{mmol})$ was added under argon atmosphere and ice-bath cooling. After $45 \mathrm{~min}$, a solution of $(S, S, S)-7 \mathbf{d}(98 \mathrm{mg}, 0.1 \mathrm{mmol})$ in dichloromethane $(1 \mathrm{ml})$ was added and the reaction mixture was stirred for 25 hours at $0{ }^{\circ} \mathrm{C}$. The reaction mixture was poured into the mixed solvent of $1 \mathrm{M}$ hydrochloric acid solution and ethyl acetate. The organic layer was washed with water, sodium hydrogen carbonate solution and brine, and dried over sodium sulfate and evaporated to give a residue. The residue was purified by PTLC (hexane/chloroform/ethyl acetate $=16 / 3 / 1$ ) to afford $(S, S, S, S, S, S, S)-8 \mathbf{d}(49 \mathrm{mg}, 50 \%$ yield) and $(S, S, S, R, S, S, S)-8 \mathbf{d}(4.6 \mathrm{mg}, 5 \%)$

$(S, S, S, S, S, S, S)-8 d$ : pale yellow foam; $[\alpha]_{\mathrm{D}}{ }^{18}=-137\left(\mathrm{c}=0.40, \mathrm{CHCl}_{3}\right)$; IR $(\mathrm{KBr}) 3504,2956,2871$, 1596, $1376 \mathrm{~cm}^{-1} ;{ }^{1} \mathrm{H}-\mathrm{NMR}\left(200 \mathrm{MHz}, \mathrm{CDCl}_{3}\right) \delta 0.55(\mathrm{t}, J=7.3,6 \mathrm{H}), 0.6-1.6(\mathrm{~m}, 50 \mathrm{H}), 3.70-4.35(\mathrm{~m}$, $16 \mathrm{H}), 3.83(\mathrm{~s}, 12 \mathrm{H}), 5.38(\mathrm{~s}, 4 \mathrm{H}), 6.36(\mathrm{~s}, 2 \mathrm{H}), 7.15-7.65(\mathrm{~m}, 42 \mathrm{H}), 7.82(\mathrm{~d}, J=7.8,2 \mathrm{H})$; FAB MS 1950 $\left(\mathrm{M}^{+}\right), 1973\left(\mathrm{M}+\mathrm{Na}^{+}\right)$; Anal. Calcd for $\mathrm{C}_{130} \mathrm{H}_{134} \mathrm{O}_{16}: \mathrm{C}, 79.97 ; \mathrm{H}, 6.92$. Found: C, 79.68; H, 7.06.

$(S, S, S, R, S, S, S)-8 d$ : pale yellow foam; $[\alpha]_{\mathrm{D}}{ }^{18}=-81\left(\mathrm{c}=0.11, \mathrm{CHCl}_{3}\right) ; \mathrm{IR}(\mathrm{KBr}) 2958,1442,1335$, $1241 \mathrm{~cm}^{-1} ;{ }^{1} \mathrm{H}-\mathrm{NMR}\left(300 \mathrm{MHz}, \mathrm{CDCl}_{3}\right) \delta 0.56(\mathrm{t}, J=7.2,6 \mathrm{H}), 0.6-1.6(\mathrm{~m}, 50 \mathrm{H}), 3.70-3.90(\mathrm{~m}, 8 \mathrm{H})$, $3.83(\mathrm{~s}, 6 \mathrm{H}), 3.86(\mathrm{~s}, 6 \mathrm{H}), 4.0-4.3(\mathrm{~m}, 8 \mathrm{H}), 5.38(\mathrm{~s}, 4 \mathrm{H}), 6.04(\mathrm{~s}, 2 \mathrm{H}), 7.1-7.65(\mathrm{~m}, 42 \mathrm{H}), 7.82(\mathrm{~d}, J=8.3$, 2H); FAB MS $1950\left(\mathrm{M}^{+}\right)$; Anal. Calcd for $\mathrm{C}_{130} \mathrm{H}_{134} \mathrm{O}_{16} \cdot 2 \mathrm{H}_{2} \mathrm{O}: \mathrm{C}, 78.52 ; \mathrm{H}, 7.00$. Found: C, 78.28; $\mathrm{H}$, 7.08 .

$(R, R, R, R, R, R, R)-8 \mathbf{b}:$ yellow foam; $[\alpha]_{\mathrm{D}}{ }^{20}=+103.2\left(\mathrm{c}=0.37, \mathrm{CHCl}_{3}\right)$; IR $(\mathrm{KBr}) 3326,1757,1598$, $1505 \mathrm{~cm}^{-1} ;{ }^{1} \mathrm{H}-\mathrm{NMR}\left(200 \mathrm{MHz}, \mathrm{CDCl}_{3}\right) \delta 1.31(\mathrm{~s}, 18 \mathrm{H}), 1.33(\mathrm{~s}, 18 \mathrm{H}), 1.36(\mathrm{~s}, 18 \mathrm{H}), 1.46(\mathrm{~s}, 18 \mathrm{H})$, $3.82(\mathrm{~s}, 6 \mathrm{H}), 3.83(\mathrm{~s}, 6 \mathrm{H}), 4.15-4.8(\mathrm{~m}, 16 \mathrm{H}), 5.39(\mathrm{~s}, 4 \mathrm{H}), 7.1-7.7(\mathrm{~m}, 42 \mathrm{H}), 7.81(\mathrm{~d}, J=7.4,2 \mathrm{H}), 8.83$ (s, 2H); Anal. Calcd for $\mathrm{C}_{146} \mathrm{H}_{150} \mathrm{O}_{32} \cdot \mathrm{H}_{2} \mathrm{O}$ : C, 72.02; H, 6.29. Found: C, 71.90; H, 6.31. 
$(R, R, R, S, R, R, R)-8 \mathbf{b}$ : yellow foam; $[\alpha]_{\mathrm{D}}{ }^{20}=+81.2\left(\mathrm{c}=0.34, \mathrm{CHCl}_{3}\right) ; \mathrm{IR}(\mathrm{KBr}) 3441,2978,1757,1598$, 1228, $1154 \mathrm{~cm}^{-1}$; ${ }^{1} \mathrm{H}-\mathrm{NMR}\left(200 \mathrm{MHz}, \mathrm{CDCl}_{3}\right) \delta 1.32(\mathrm{~s}, 36 \mathrm{H}), 1.37(\mathrm{~s}, 18 \mathrm{H}), 1.44(\mathrm{~s}, 18 \mathrm{H}), 3.78(\mathrm{~s}, 6 \mathrm{H})$, $3.85(\mathrm{~s}, 6 \mathrm{H}), 4.2-4.8(\mathrm{~m}, 16 \mathrm{H}), 5.38(\mathrm{~s}, 4 \mathrm{H}), 7.2-7.6(\mathrm{~m}, 42 \mathrm{H}), 7.81(\mathrm{~d}, J=8.6,2 \mathrm{H}), 8.46(\mathrm{~s}, 2 \mathrm{H}) ;$ Anal. Calcd for $\mathrm{C}_{146} \mathrm{H}_{150} \mathrm{O}_{32} \cdot 1.5 \mathrm{H}_{2} \mathrm{O}$ : C, 71.76; H, 6.31. Found: C, 71.84; H, 6.38.

$(S, S, S, S, S, S, S)-8 \mathrm{c}:$ white powder; $\mathrm{Mp}, 156^{\circ} \mathrm{C} ;[\alpha]_{\mathrm{D}}{ }^{21}=-96.5\left(\mathrm{c}=0.77, \mathrm{CHCl}_{3}\right)$; $\mathrm{IR}(\mathrm{KBr}) 2977,1645$, 1457, 1249, $1118 \mathrm{~cm}^{-1} ;{ }^{1} \mathrm{H}-\mathrm{NMR}\left(200 \mathrm{MHz}, \mathrm{CDCl}_{3}\right) \delta$ 0.50-1.20 (m, 48H), 2.60-3.50 (m, 32H), $3.89(\mathrm{~s}$, 6H), $3.92(\mathrm{~s}, 6 \mathrm{H}), 4.30-4.90(\mathrm{~m}, 16 \mathrm{H}), 5.37(\mathrm{~s}, 4 \mathrm{H}), 7.10-7.70(\mathrm{~m}, 42 \mathrm{H}), 7.84(\mathrm{~d}, J=8.0,2 \mathrm{H}), 10.73(\mathrm{~s}$, 2H); FAB MS $2407\left(\mathrm{M}^{+}\right), 2430\left(\mathrm{M}+\mathrm{Na}^{+}\right)$.

$(S, S, S, R, S, S, S)-8 \mathbf{8}:$ : white powder; $\mathrm{Mp}, 140{ }^{\circ} \mathrm{C} ;[\alpha]_{\mathrm{D}}{ }^{21}=-80.3\left(\mathrm{c}=0.50, \mathrm{CHCl}_{3}\right) ; \mathrm{IR}(\mathrm{KBr}) 2976$, $1637,1257,1399,1251,1117 \mathrm{~cm}^{-1} ;{ }^{1} \mathrm{H}-\mathrm{NMR}\left(200 \mathrm{MHz}, \mathrm{CDCl}_{3}\right) \delta 0.63(\mathrm{t}, J=7.0,12 \mathrm{H}), 0.75-1.10(\mathrm{~m}$, $36 \mathrm{H}), 2.55-2.90(\mathrm{~m}, 8 \mathrm{H}), 2.90-3.50(\mathrm{~m}, 24 \mathrm{H}), 3.90(\mathrm{~s}, 12 \mathrm{H}), 4.35-4.90(\mathrm{~m}, 16 \mathrm{H}), 5.37(\mathrm{~s}, 4 \mathrm{H}), 7.10-7.65$ (m, 42H), $7.84(\mathrm{~d}, J=8.0,2 \mathrm{H}), 10.59(\mathrm{~s}, 2 \mathrm{H}) ; \mathrm{FAB} \mathrm{MS} 2407\left(\mathrm{M}^{+}\right), 2430\left(\mathrm{M}+\mathrm{Na}^{+}\right)$. 


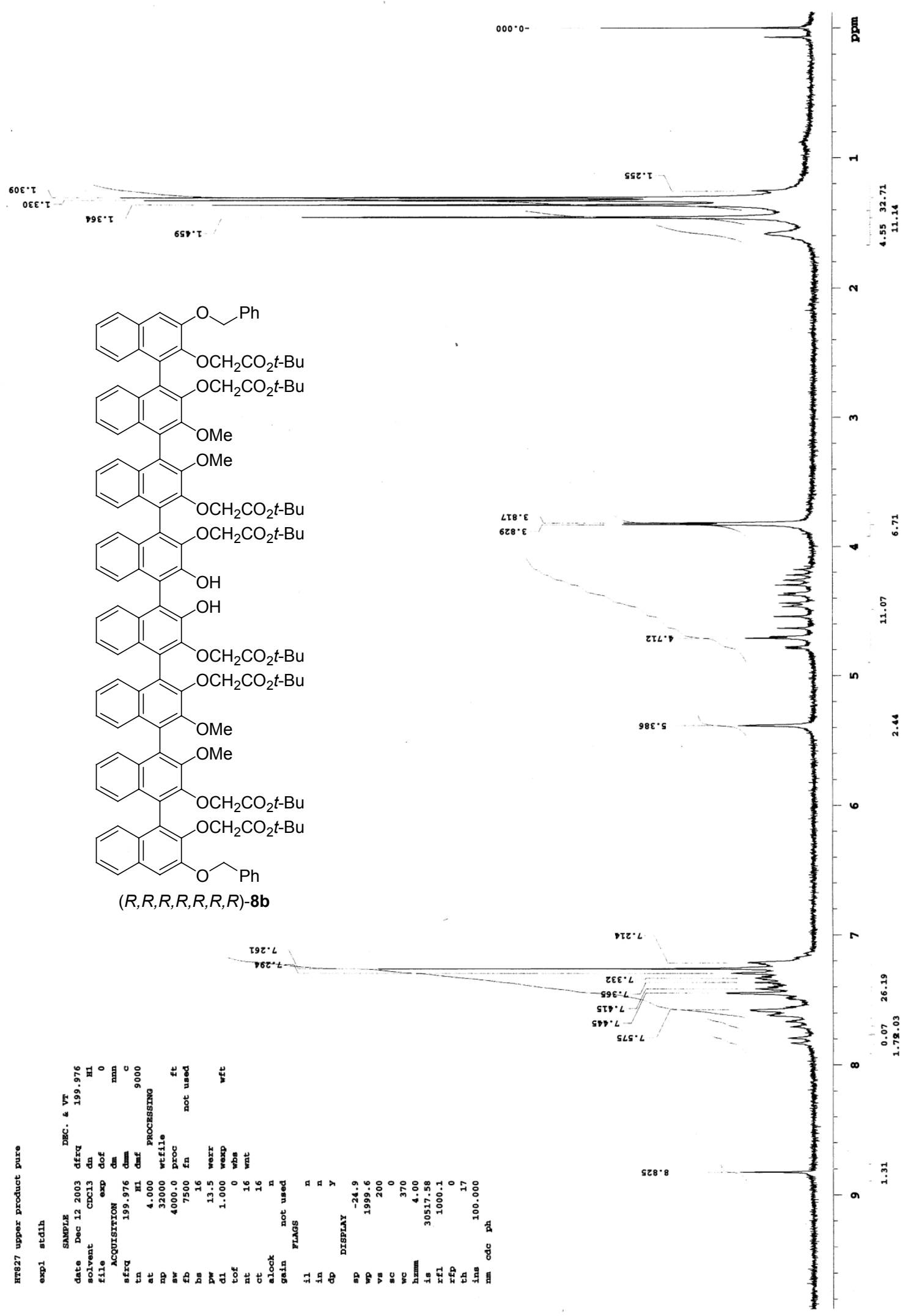




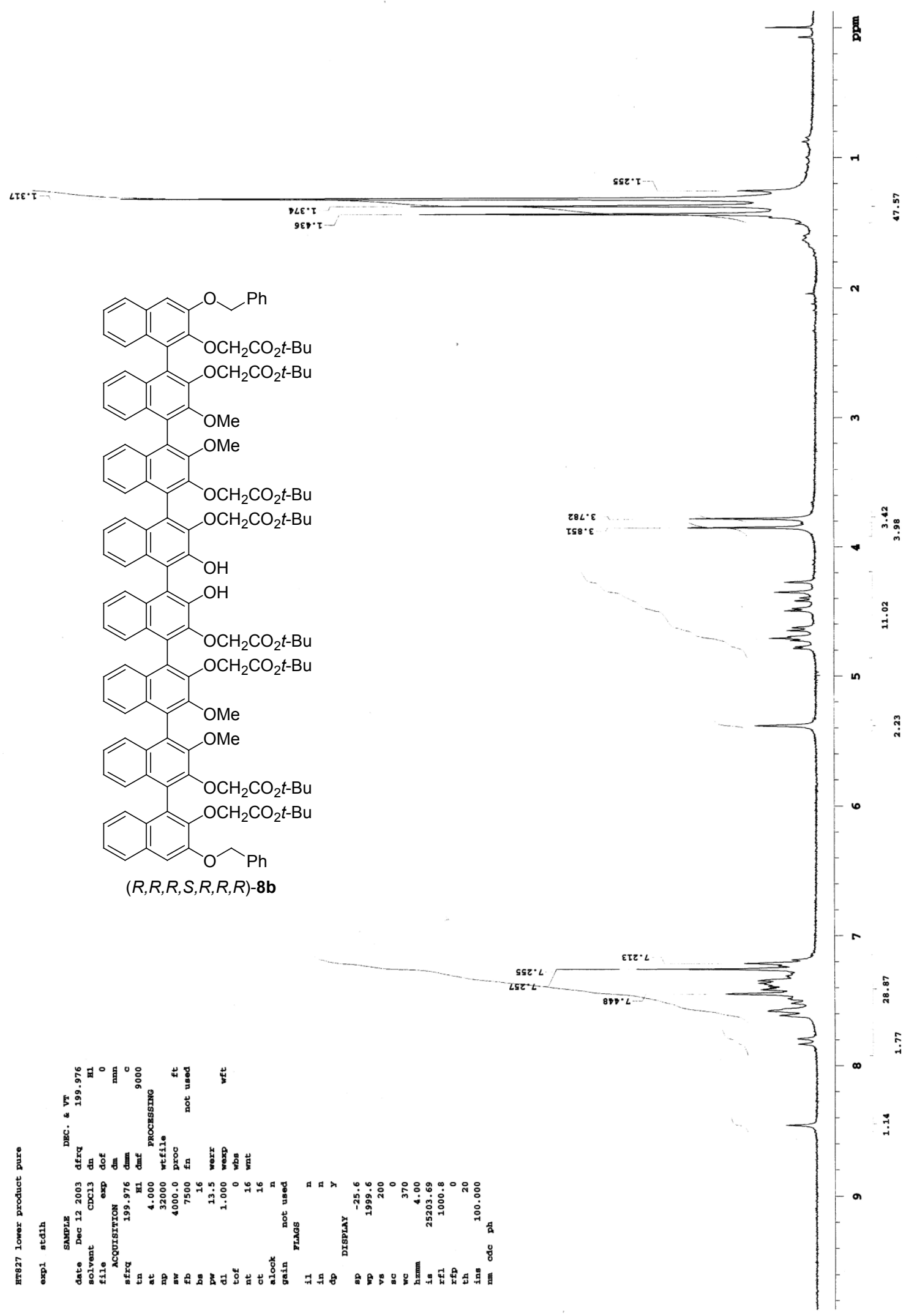




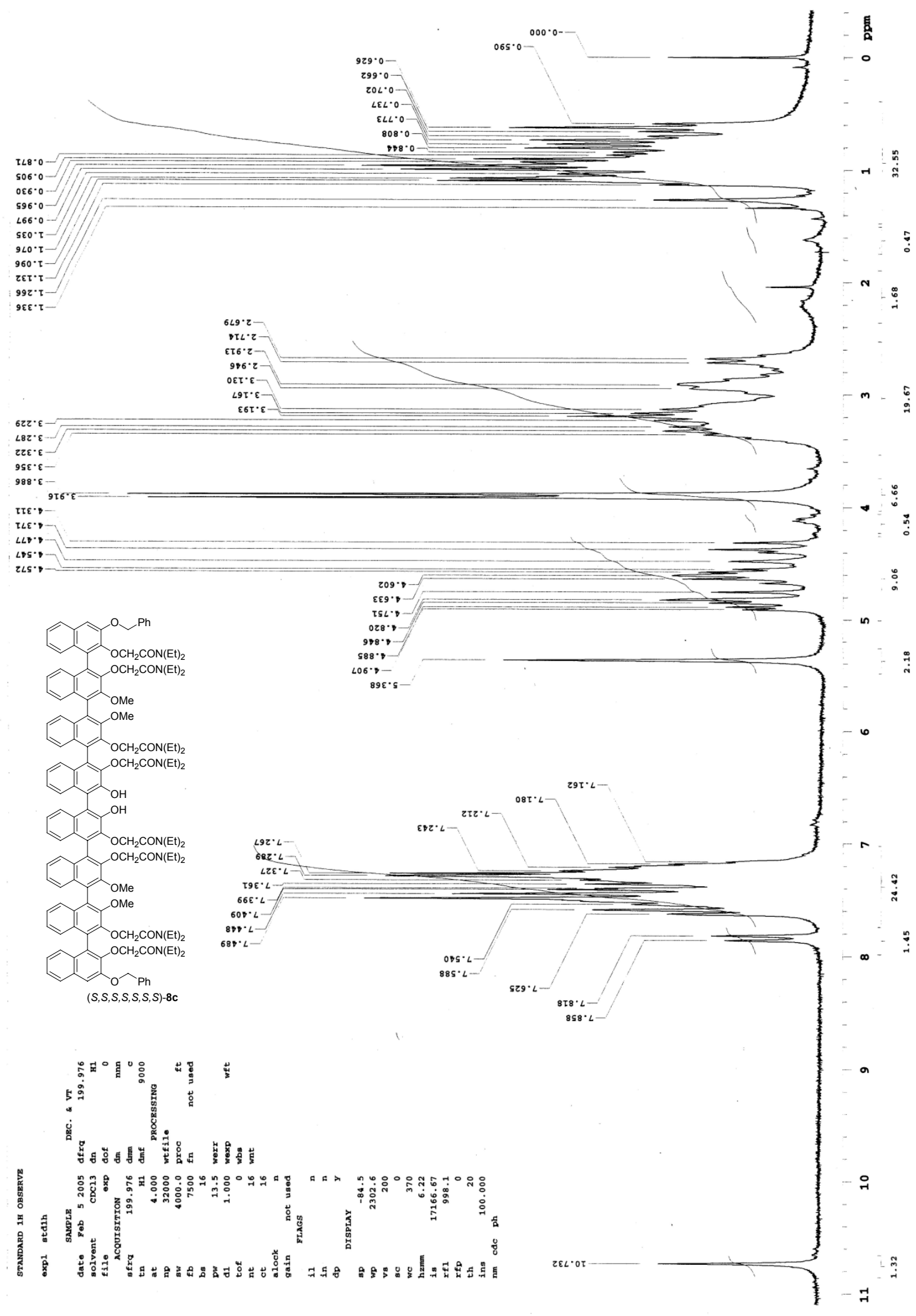




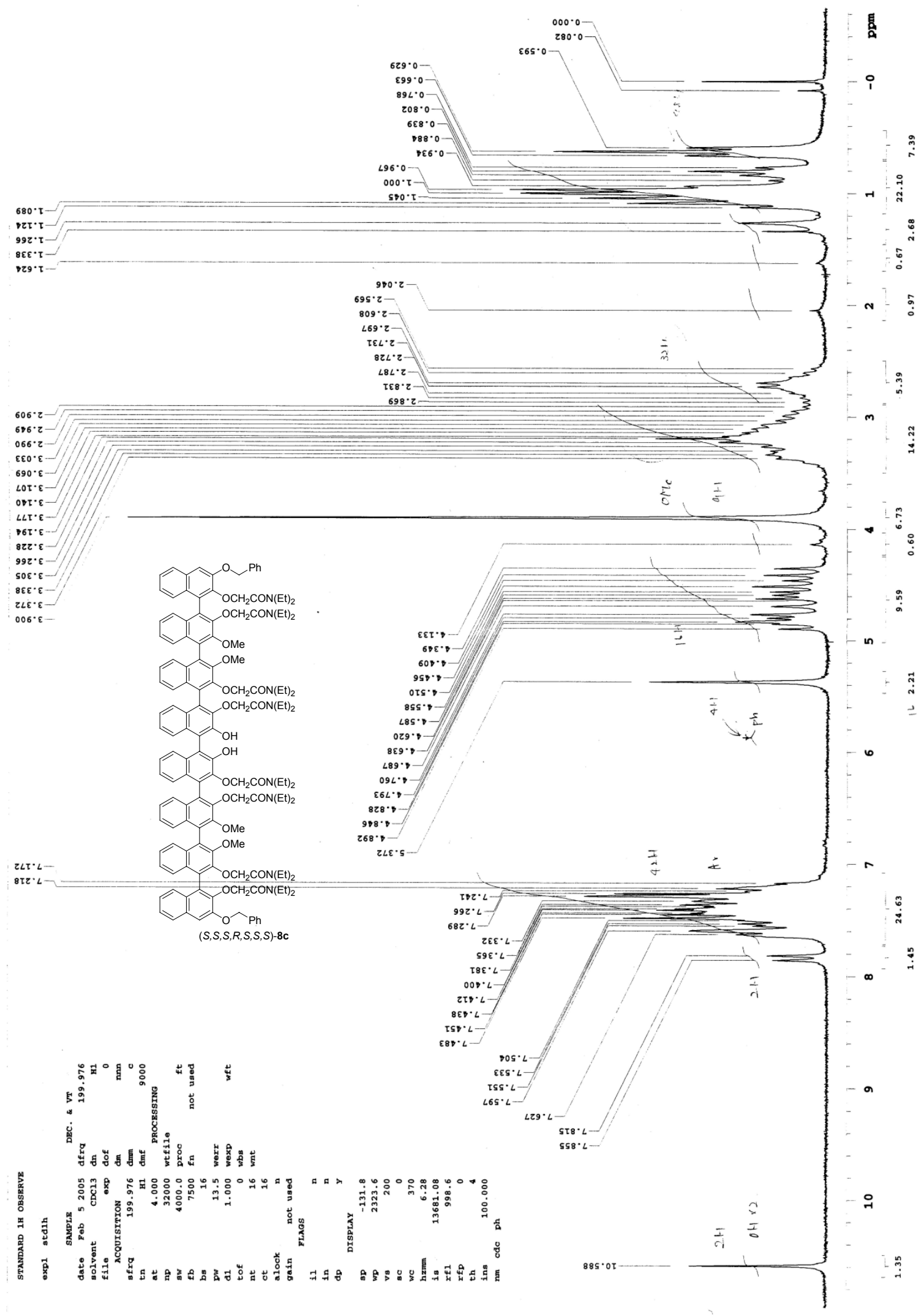




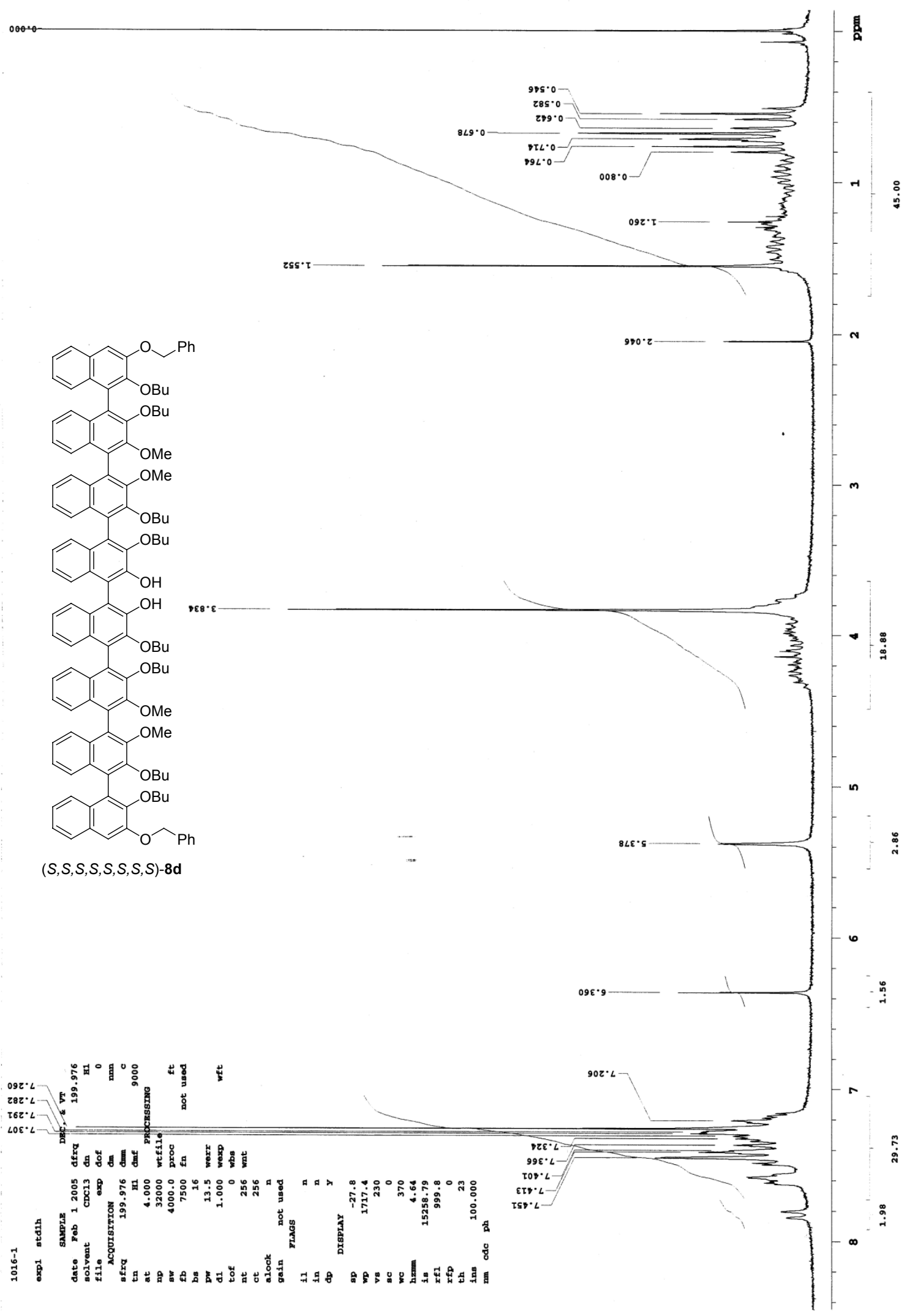




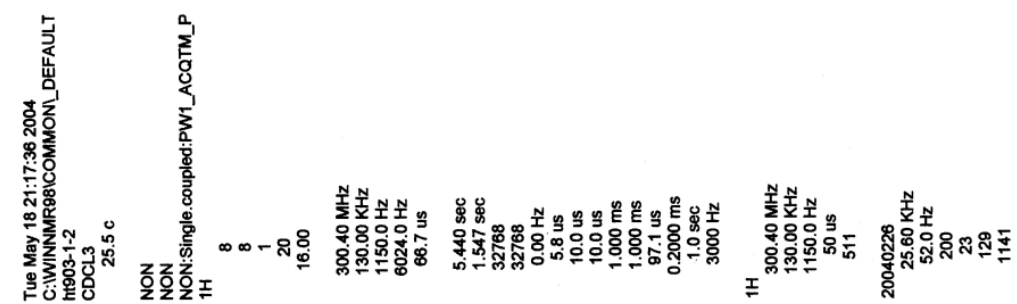

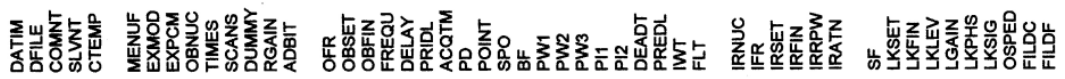

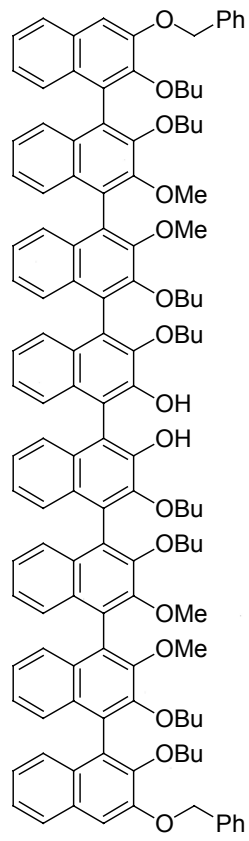

$(S, S, S, S, R, S, S, S)-8 \mathbf{d}$ 


\section{Synthesis of Compounds $(S, S, S)-2 \mathrm{c}-\mathrm{d}_{14}$}

(S)-33c $\longrightarrow$

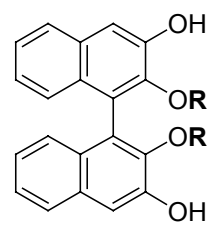

(S)-36
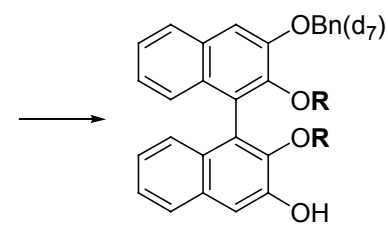

(S)-37

$\mathrm{R}=\mathrm{CH}_{2} \mathrm{CON}(\mathrm{Et})_{2}$

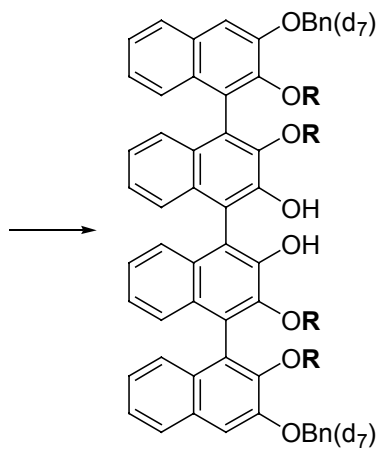

$(S, S, S)-2 c-d_{14}$

(S)-36: $(S)$-36 was isolated as a side product of reaction 33c to $1 \mathbf{c}$. white powder; $\mathrm{Mp}, 173-174{ }^{\circ} \mathrm{C}$, $[\alpha]_{\mathrm{D}}{ }^{20}=+98.6\left(\mathrm{c}=1.01, \mathrm{CHCl}_{3}\right) ; \mathrm{IR}(\mathrm{KBr}) 2980,1638,1509,1443 \mathrm{~cm}^{-1} ;{ }^{1} \mathrm{H}-\mathrm{NMR}\left(200 \mathrm{MHz}, \mathrm{CDCl}_{3}\right) \delta$ $0.76(\mathrm{t}, J=7.2,6 \mathrm{H}), 1.06(\mathrm{t}, J=7.1,6 \mathrm{H}), 2.66(\mathrm{q}, J=7.2,4 \mathrm{H}), 3.1-3.5(\mathrm{~m}, 4 \mathrm{H}), 4.04(\mathrm{~d}, J=16.0,2 \mathrm{H})$, $4.22(\mathrm{~d}, J=16.0,2 \mathrm{H}), 7.0-7.15(\mathrm{~m}, 4 \mathrm{H}), 7.3-7.4(\mathrm{~m}, 2 \mathrm{H}), 7.49(\mathrm{~s}, 2 \mathrm{H}), 7.76(\mathrm{~d}, J=8.2,2 \mathrm{H}), 10.76$ (brs, $2 \mathrm{H}$ ); HRMS (EI) Calcd for $\mathrm{C}_{32} \mathrm{H}_{36} \mathrm{O}_{6} \mathrm{~N}_{2}$ : 544.2573. Found: 544.2568; Anal. Calcd for $\mathrm{C}_{32} \mathrm{H}_{36} \mathrm{O}_{6} \mathrm{~N}_{2}$ : C, 70.57; H, 6.66; N, 5.14. Found: C, 70.58; H, 6.71; N, 5.13.

$(S)-37$ : Benzyl-d 7 bromide $(107 \mu \mathrm{l}, 0.9 \mathrm{mmol})$ was added to a suspension of $(S)-36(1.0 \mathrm{~g}, 1.8 \mathrm{mmol})$ and $\mathrm{K}_{2} \mathrm{CO}_{3}(124 \mathrm{mg}, 0.9 \mathrm{mmol})$ in acetone $(10 \mathrm{ml})$. The suspension was stirred for 2 days at room temperature. The reaction mixture was poured into the mixed solvent of ethyl acetate and hydrochloric acid solution. After dried over sodium sulfate, the solvent was evaporated in vacuo to give a residue. The residue was purified by column chromatography $\left(\mathrm{SiO}_{2}\right.$, hexane/ethyl acetate $\left.=2 / 1\right)$ to afford $(S)-37$ $(275 \mathrm{mg}, 48 \%)$ as white powder. white powder; Mp, $173-174{ }^{\circ} \mathrm{C} ;[\alpha]_{\mathrm{D}}^{18}=-10\left(\mathrm{c}=0.23, \mathrm{CHCl}_{3}\right) ; \mathrm{IR}^{18}$ (KBr) 2973, 1639, 1437, $1246 \mathrm{~cm}^{-1} ;{ }^{1} \mathrm{H}-\mathrm{NMR}\left(300 \mathrm{MHz}, \mathrm{CDCl}_{3}\right) \delta 0.54(\mathrm{t}, J=7.1,3 \mathrm{H}), 0.83(\mathrm{t}, J=7.2$, $3 \mathrm{H}), 0.90(\mathrm{t}, J=7.1,3 \mathrm{H}), 1.06(\mathrm{t}, J=7.1,3 \mathrm{H}), 2.50-3.45(\mathrm{~m}, 8 \mathrm{H}), 4.13(\mathrm{~d}, J=15.9,1 \mathrm{H}), 4.29(\mathrm{~d}, J=$ $11.7,1 \mathrm{H}), 4.39(\mathrm{~d}, J=16.2,1 \mathrm{H}), 4.64(\mathrm{~d}, J=11.7,1 \mathrm{H}), 7.05-7.20(\mathrm{~m}, 4 \mathrm{H}), 7.25-7.40(\mathrm{~m}, 2 \mathrm{H}), 7.44(\mathrm{~s}$, $2 \mathrm{H}), 7.72(\mathrm{~d}, J=8.4,1 \mathrm{H}), 7.77(\mathrm{~d}, J=7.8,1 \mathrm{H}), 10.71(\mathrm{~s}, 1 \mathrm{H}) ; \mathrm{HRMS}(\mathrm{EI})$ Calcd for $\mathrm{C}_{38} \mathrm{H}_{35} \mathrm{D}_{7} \mathrm{O}_{6} \mathrm{~N}_{2}$ : 641.3483. Found: 641.3491; Anal. Calcd for $\mathrm{C}_{38} \mathrm{H}_{35} \mathrm{D}_{7} \mathrm{O}_{6} \mathrm{~N}_{2}$ : C, 72.98; $\mathrm{H}+\mathrm{D}, 7.70 ; \mathrm{N}, 4.36$. Found: C, $72.73 ; \mathrm{H}+\mathrm{D}, 7.70 ; \mathrm{N}, 4.39$. 
$(S, S, S)-2 \mathrm{c}-\mathrm{d}_{14}: \quad$ To a mixture of $\mathrm{CuCl}_{2}(108 \mathrm{mg}, 0.8 \mathrm{mmol})$ in methanol $(2 \mathrm{ml})$, $(d l)-\alpha-$ phenylethylamine $(128 \mu \mathrm{l}, 1.0 \mathrm{mmol})$ was added under argon atmosphere at room temperature. After 45 min, a solution of $(S)-37(256 \mathrm{mg}, 0.4 \mathrm{mmol})$ in dichloromethane $(2 \mathrm{ml})$ was added and the reaction mixture was stirred for 18 hours at $0{ }^{\circ} \mathrm{C}$. The reaction mixture was poured into the mixed solvent of 1 M hydrochloric acid solution and ethyl acetate. The organic layer was washed with water (three times) and brine, and dried over sodium sulfate and evaporated to give a residue. The residue was purified by column chromatography $\left(\mathrm{SiO}_{2}\right.$ hexane/chloroform/ethyl acetate $\left.=1 / 1 / 1\right)$ and by PTLC (hexane/acetone $=1 / 1)$ to afford to $(S, S, S)-\mathbf{2} \mathbf{c}-\mathrm{d}_{14}(79 \mathrm{mg}, 31 \%)$. colorless foam; $[\alpha]_{\mathrm{D}}{ }^{18}=-35\left(\mathrm{c}=0.49, \mathrm{CHCl}_{3}\right) ; \mathrm{IR}^{2}$ (KBr) 3446, 2934, 1639, 1441, $1245 \mathrm{~cm}^{-1} ;{ }^{1} \mathrm{H}-\mathrm{NMR}\left(300 \mathrm{MHz}, \mathrm{CDCl}_{3}\right) \delta 0.68(\mathrm{t}, J=7.1,6 \mathrm{H}), 0.81(\mathrm{t}$, $J=7.1,6 \mathrm{H}), 0.92-1.06(\mathrm{~m}, 12 \mathrm{H}), 2.65-3.00(\mathrm{~m}, 8 \mathrm{H}), 3.05-3.40(\mathrm{~m}, 8 \mathrm{H}), 4.16(\mathrm{~d}, J=15.9,2 \mathrm{H}), 4.33(\mathrm{~d}$, $J=15.9,2 \mathrm{H}), 4.49(\mathrm{~d}, J=12.0,2 \mathrm{H}), 4.76(\mathrm{~d}, J=12.3,2 \mathrm{H}), 7.00-7.50(\mathrm{~m}, 16 \mathrm{H}), 7.80(\mathrm{~d}, J=7.8,2 \mathrm{H})$, 10.52 (s, 2H); HRMS (FAB) Calcd for $\mathrm{C}_{78} \mathrm{H}_{68} \mathrm{D}_{14} \mathrm{~N}_{4} \mathrm{O}_{12}$ : 1280.6808. Found: 1280.6786; Anal. Calcd for $\mathrm{C}_{78} \mathrm{H}_{68} \mathrm{D}_{14} \mathrm{~N}_{4} \mathrm{O}_{12} \cdot 1 \mathrm{H}_{2} \mathrm{O}: \mathrm{C}, 72.09 ; \mathrm{H}+\mathrm{D}, 7.60 ; \mathrm{N}, 4.31$. Found: $\mathrm{C}, 72.33 ; \mathrm{H}+\mathrm{D}, 7.66 ; \mathrm{N}, 4.36$. 


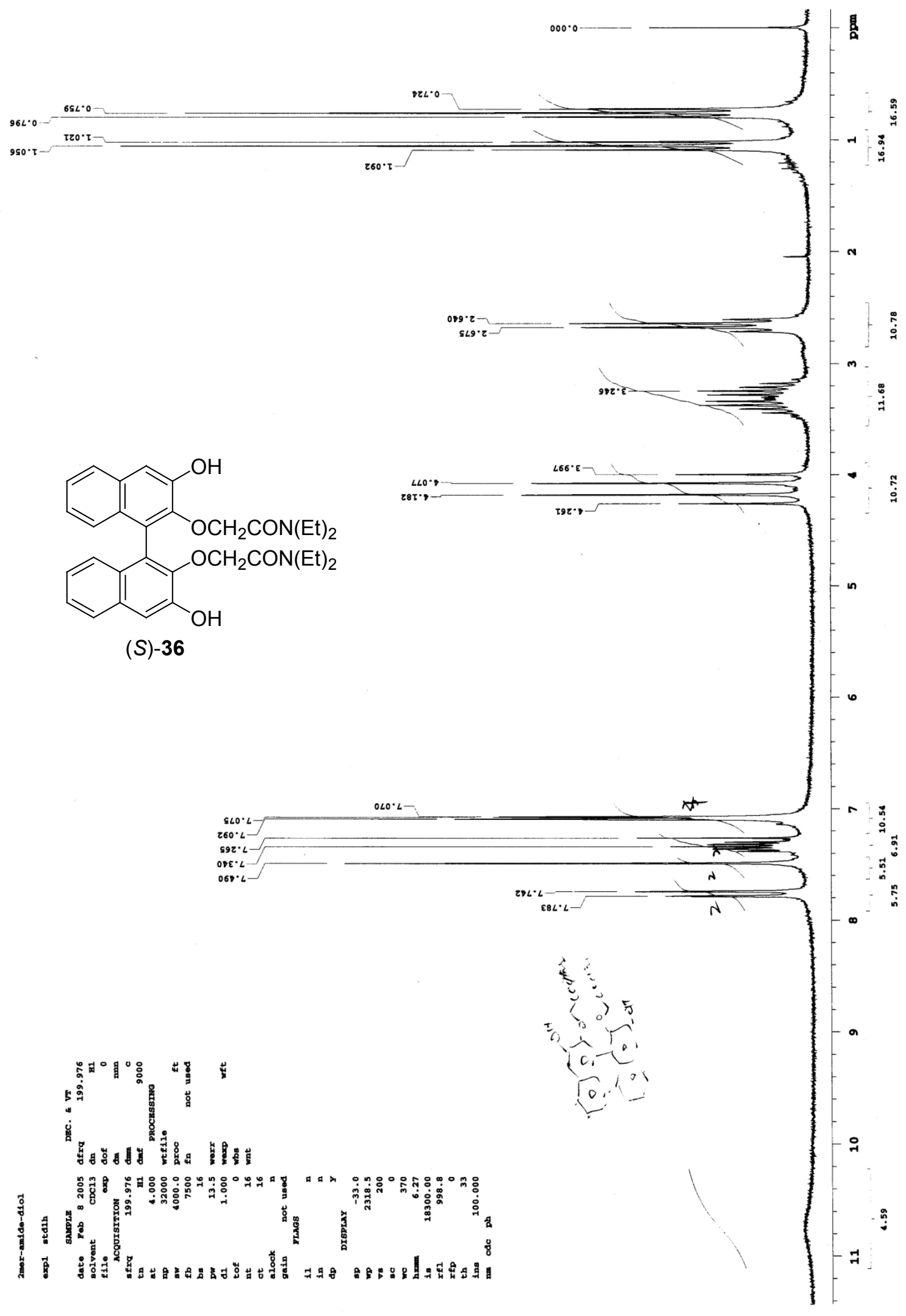



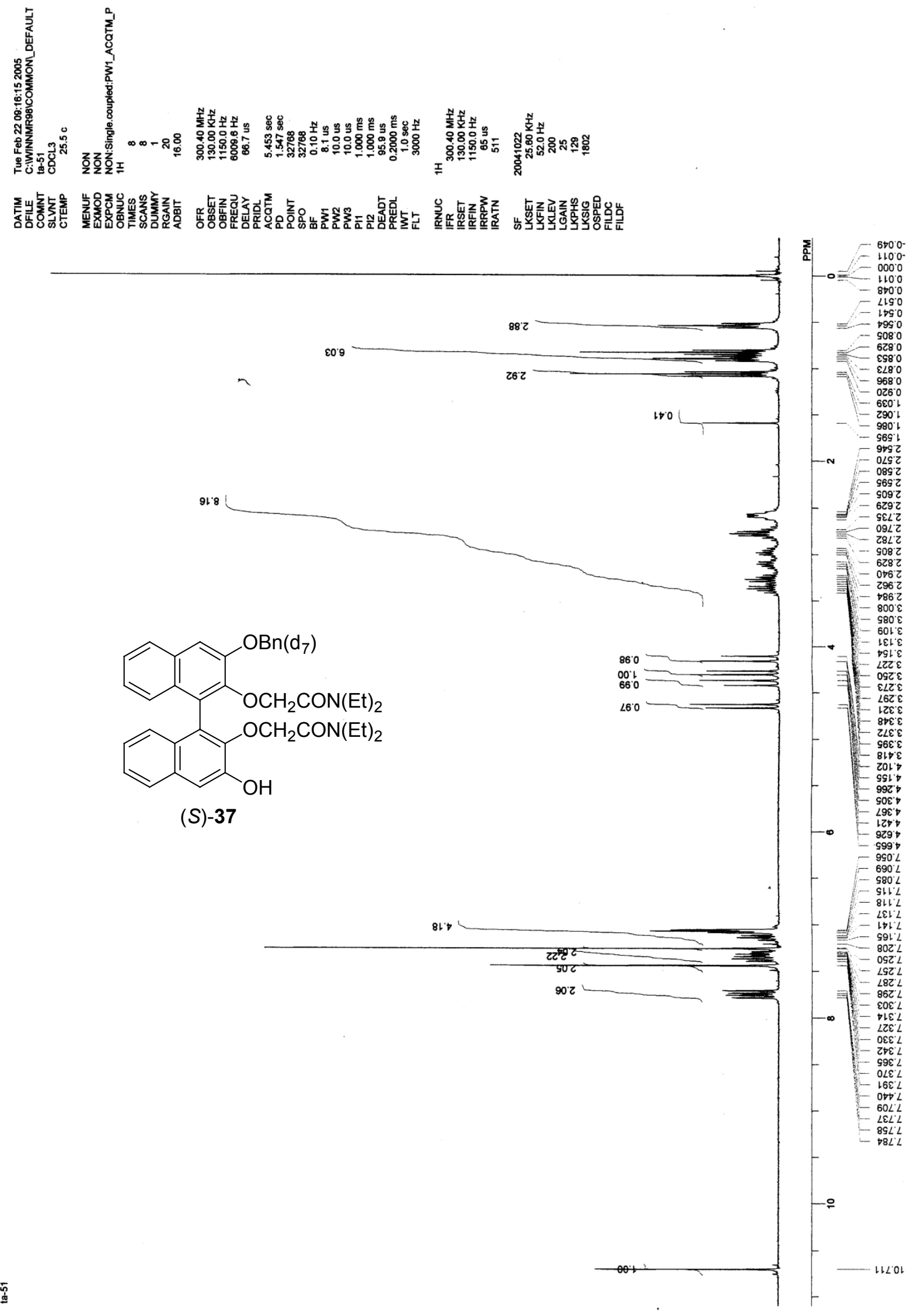


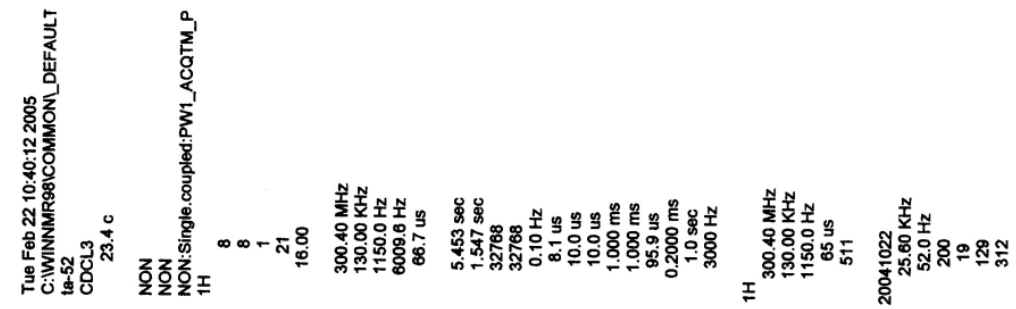

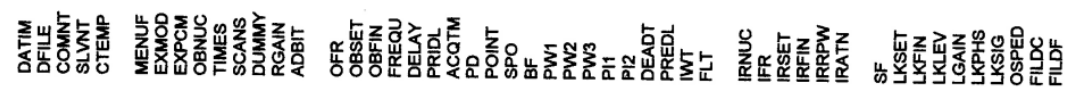

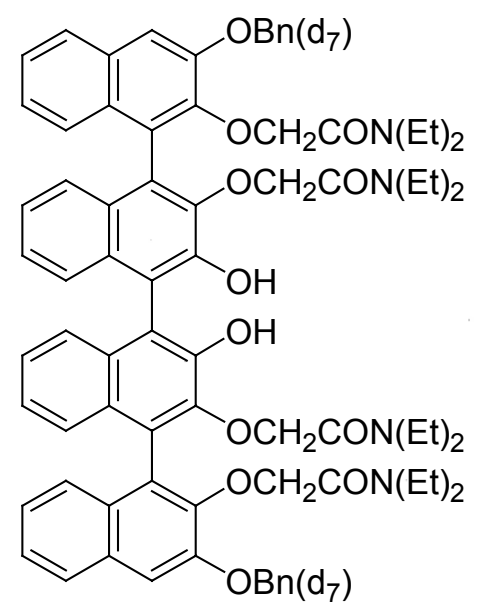

$(S, S, S)-2 c-d_{14}$

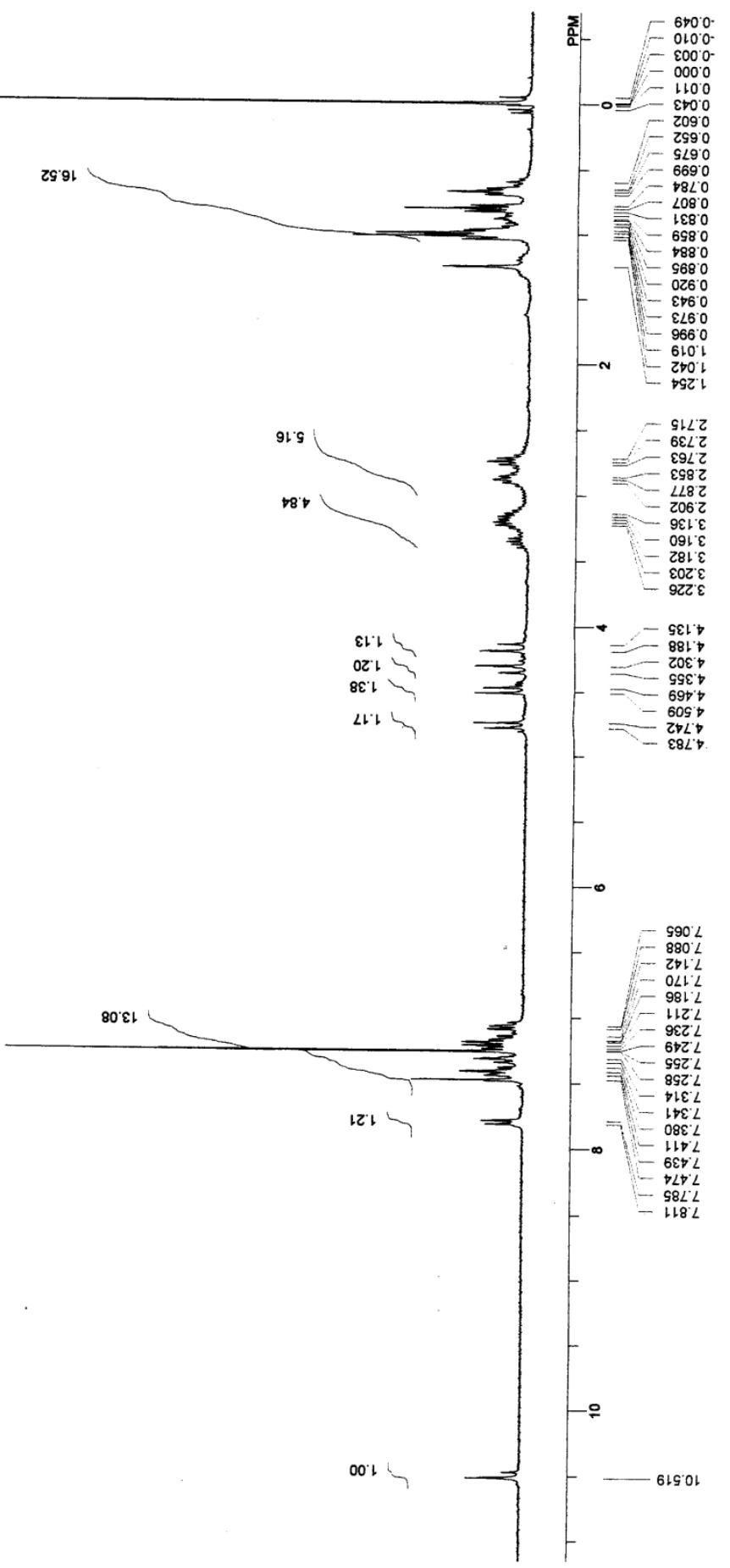




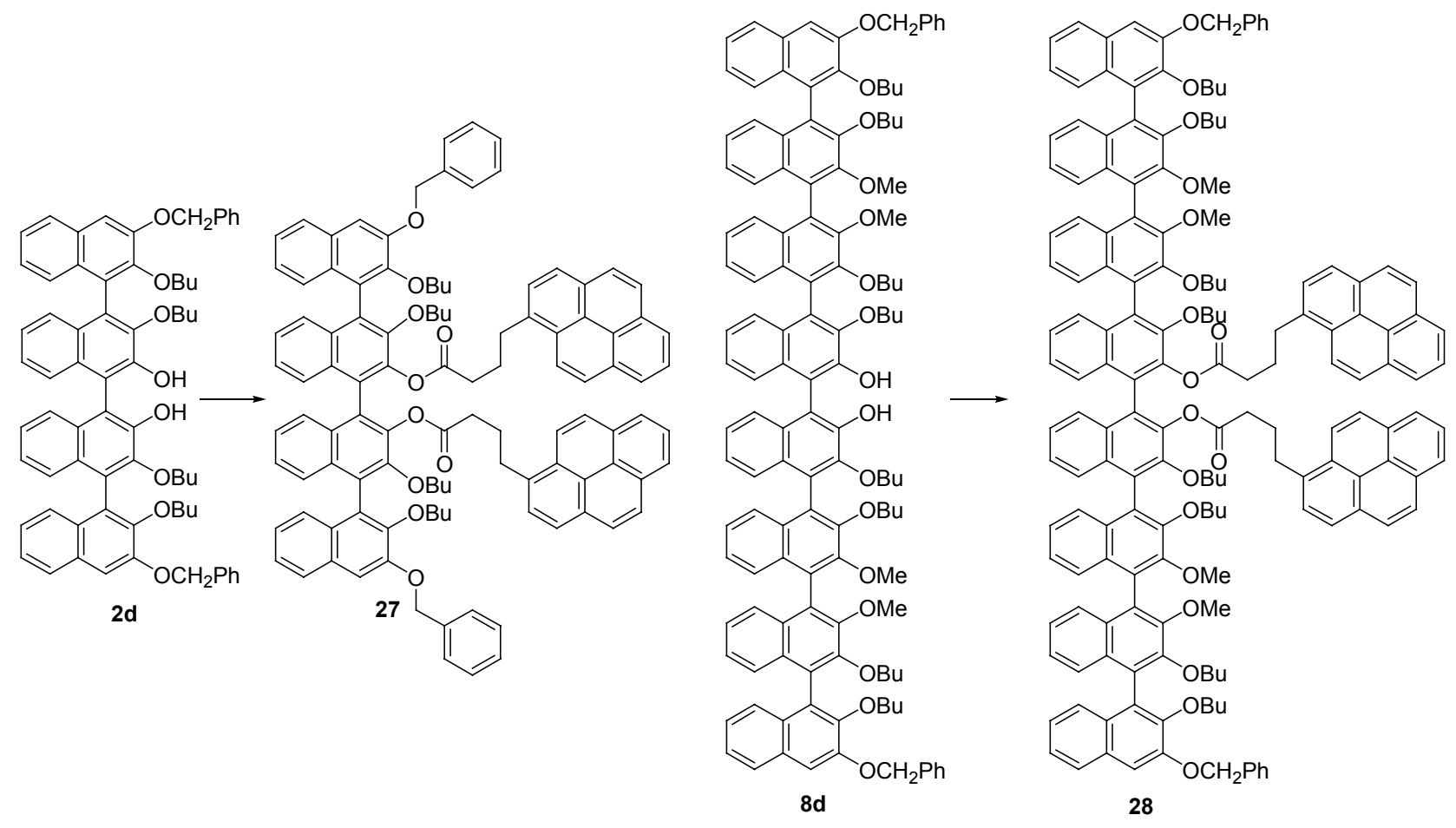

General Procedure for the Synthesis of Pyrene Compounds 27 and 28:

The synthesis of $(S, S, S)-\mathbf{2 7}$ is typical.

To a solution of $(S, S, S)-2 \mathbf{d}(25 \mathrm{mg}, 0.024 \mathrm{mmol}), 1$-pyrenebutyric acid $(21 \mathrm{mg}, 0.072 \mathrm{mmol})$ and DMAP (3 mg) in DMF (4.0 ml), WSC $\cdot \mathrm{HCl}(14 \mathrm{mg}, 0.072 \mathrm{mmol})$ was added and the solution was stirred for 1 day at room temperature. The reaction mixture was poured into chloroform and $0.1 \mathrm{M}$ hydrochloric acid. The organic layer was separated, washed successively with water (twice), brine, and dried over sodium sulfate and evaporated in vacuo. The residue was purified by PTLC (hexane/ethyl acetate $=3 / 1)$ to afford $(S, S, S)-27(34 \mathrm{mg}, 92 \%) . \mathrm{Mp} ; 87^{\circ} \mathrm{C} ;[\alpha]_{\mathrm{D}}{ }^{21}=-33.6\left(\mathrm{c}=0.80, \mathrm{CHCl}_{3}\right) ; \mathrm{IR}(\mathrm{KBr})$ 3009, 1761, 1442, $1376 \mathrm{~cm}^{-1} ;{ }^{1} \mathrm{H}-\mathrm{NMR}\left(200 \mathrm{MHz}, \mathrm{CDCl}_{3}\right) \delta 0.38(\mathrm{t}, J=7.4 \mathrm{~Hz}, 6 \mathrm{H}), 0.72(\mathrm{t}, J=7.4$ $\mathrm{Hz}, 6 \mathrm{H}), 0.6-1.6(\mathrm{~m}, 16 \mathrm{H}), 1.9-2.2(\mathrm{~m}, 4 \mathrm{H}), 2.4-2.6(\mathrm{~m}, 4 \mathrm{H}), 2.9-3.3(\mathrm{~m}, 4 \mathrm{H}), 3.4-3.6(\mathrm{~m}, 2 \mathrm{H}), 3.7-4.0$ (m, 4H), 4.1-4.3 (m, 2H), $5.35(\mathrm{~s}, 4 \mathrm{H}), 6.77(\mathrm{t}, J=7.0 \mathrm{~Hz}, 2 \mathrm{H}), 7.10-7.65(\mathrm{~m}, 26 \mathrm{H}), 7.7-8.2(\mathrm{~m}, 18 \mathrm{H})$; HRMS (FAB) Calcd for $\mathrm{C}_{110} \mathrm{H}_{98} \mathrm{O}_{10}: 1578.7160$. Found: 1578.7177

$(S, R, S)-27: \mathrm{Mp} ; 89^{\circ} \mathrm{C} ;[\alpha]_{\mathrm{D}}^{21}=-16.7\left(\mathrm{c}=0.70, \mathrm{CHCl}_{3}\right)$; IR $(\mathrm{KBr}) 3008,2959,1763,1442 \mathrm{~cm}^{-1} ;{ }^{1} \mathrm{H}-$ $\operatorname{NMR}\left(200 \mathrm{MHz}, \mathrm{CDCl}_{3}\right) \delta 0.35(\mathrm{t}, J=7.2 \mathrm{~Hz}, 6 \mathrm{H}), 0.60(\mathrm{t}, J=7.6 \mathrm{~Hz}, 6 \mathrm{H}), 0.7-0.9(\mathrm{~m}, 4 \mathrm{H}), 0.9-1.2$ 
(m, 8H), 1.2-1.6 (m, 4H), 1.9-2.2 (m, 4H), 2.3-2.6 (m, 4H), 3.0-3.3 (m, 4H), 3.4-3.6 (m, 2H), 3.6-3.8 (m, 2H), 3.8-4.1 (m, 2H), 4.1-4.3 (m, 2H), $5.38(\mathrm{~s}, 4 \mathrm{H}), 7.0-8.3(\mathrm{~m}, 46 \mathrm{H})$; HRMS (FAB) Calcd for $\mathrm{C}_{110} \mathrm{H}_{98} \mathrm{O}_{10}: 1578.7160$. Found: 1578.7194

$(S, S, S, S, S, S, S)-\mathbf{2 8}: \mathrm{Mp} ; 109^{\circ} \mathrm{C} ;[\alpha]_{\mathrm{D}}^{21}=-109\left(\mathrm{c}=0.45, \mathrm{CHCl}_{3}\right) ; \mathrm{IR}(\mathrm{KBr}) 2957,1764,1447,1376 \mathrm{~cm}^{-}$ '; ${ }^{1} \mathrm{H}-\mathrm{NMR}\left(200 \mathrm{MHz}, \mathrm{CDCl}_{3}\right) \delta$ 0.4-1.6 (m, 56H), 2.0-2.2 (m, 4H), 2.4-2.7 (m, 4H), 2.9-3.4 (m, 4H), 3.6-4.4 (m, 16H), $3.79(\mathrm{~s}, 6 \mathrm{H}), 3.81(\mathrm{~s}, 6 \mathrm{H}), 5.37(\mathrm{~s}, 4 \mathrm{H}), 6.7-6.9(\mathrm{~m}, 2 \mathrm{H}), 6.9-7.7(\mathrm{~m}, 42 \mathrm{H}), 7.7-8.3(\mathrm{~m}$, $18 \mathrm{H}) ; \mathrm{MS}(\mathrm{FAB}) m / z 2491\left(\mathrm{M}^{+}\right)$ 


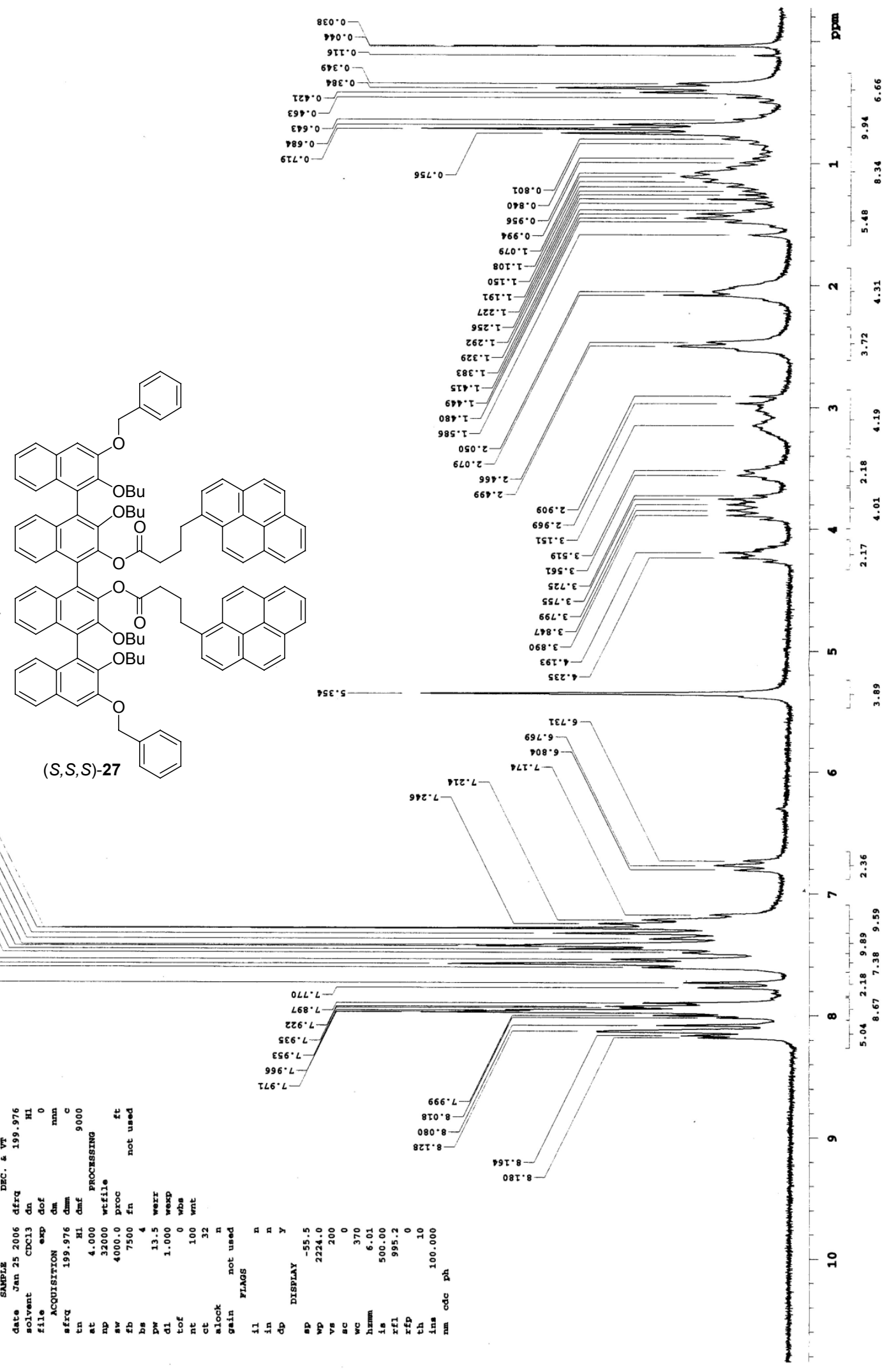




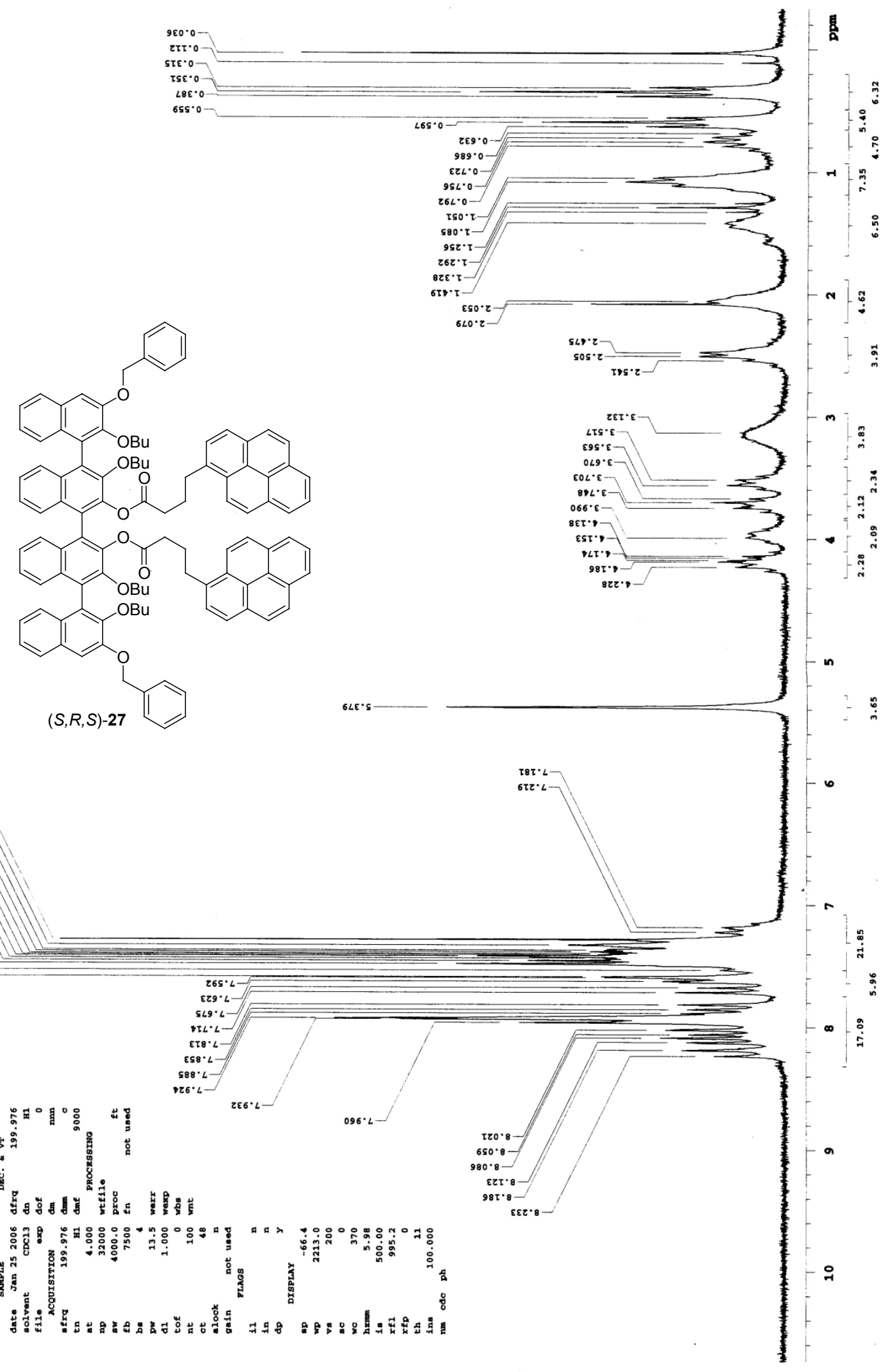



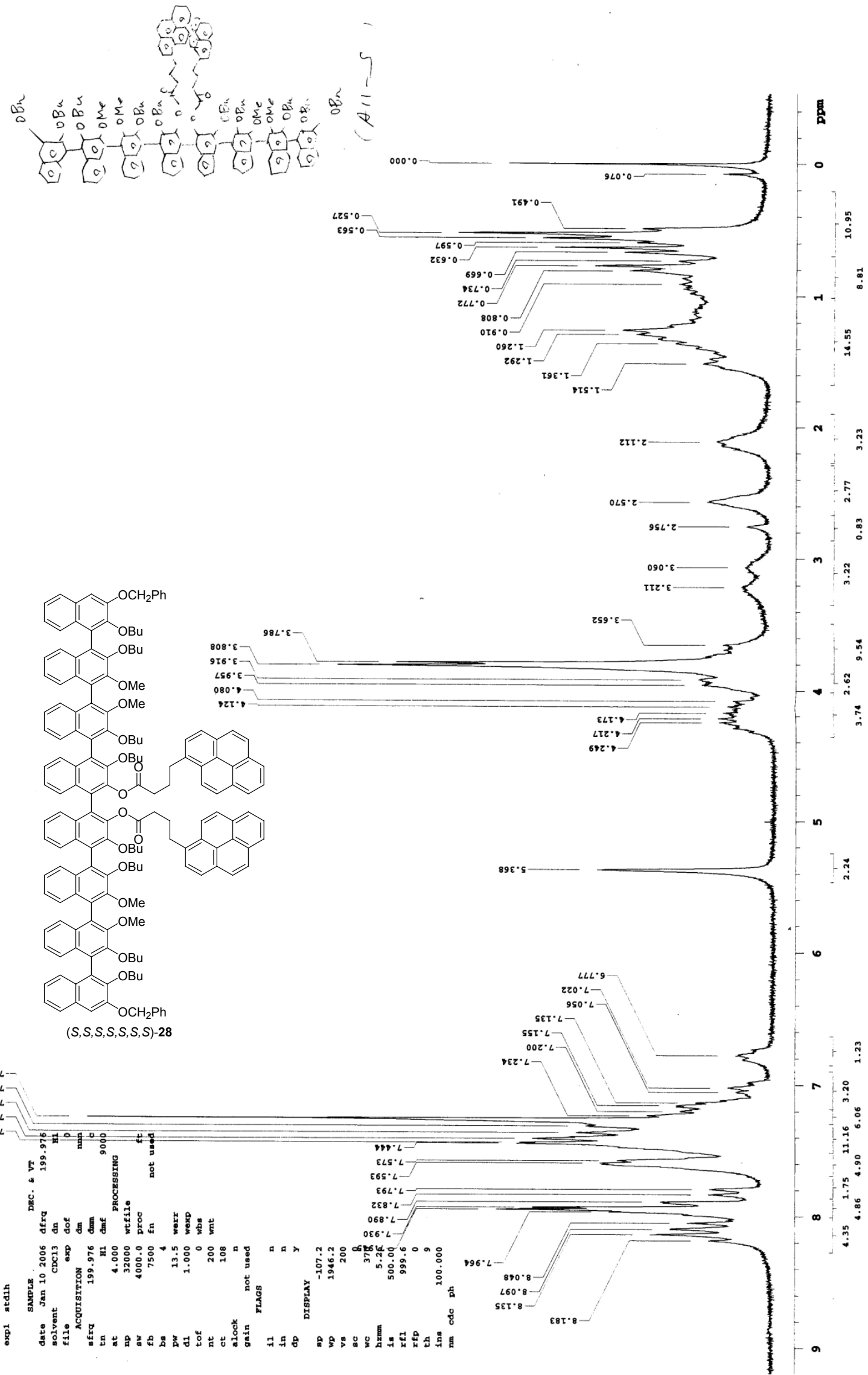


\section{General Procedure for the Synthesis of ${ }^{13} \mathrm{C}$ enrich Compounds 29 - 31:}

The synthesis of $(S)-29$ is typical.

To a suspension of $(S)-32(51 \mathrm{mg}, 0.1 \mathrm{mmol})$ and potassium carbonate $(41 \mathrm{mg}, 0.3 \mathrm{mmol})$ in DMF $(2.5$ $\mathrm{ml})$, iodomethane- ${ }^{13} \mathrm{C}(31 \mu \mathrm{l}, 0.5 \mathrm{mmol})$ was added. The reaction mixture was stirred for 15 hours at room temperature. The reaction mixture was poured into the mixed solvent of ethyl acetate and $0.1 \mathrm{M}$ hydrochloric acid solution. The organic layer was separated, washed successively with water (twice), and brine. After dried over sodium sulfate, the solvent was evaporated in vacuo to give $(S)$-29 (56 mg, 100\%). ${ }^{1} \mathrm{H}-\mathrm{NMR}\left(200 \mathrm{MHz}, \mathrm{CDCl}_{3}\right) \delta 3.66(\mathrm{~d}, J=144.4,6 \mathrm{H}), 5.33(\mathrm{~s}, 4 \mathrm{H}), 7.0-7.15(\mathrm{~m}, 4 \mathrm{H}), 7.20-$ $7.50(\mathrm{~m}, 10 \mathrm{H}), 7.50-7.60(\mathrm{~m}, 4 \mathrm{H}), 7.77(\mathrm{~d}, J=8.4 \mathrm{~Hz}, 2 \mathrm{H})$; HRMS (EI) Calcd for ${ }^{12} \mathrm{C}_{34}{ }^{13} \mathrm{C}_{2} \mathrm{H}_{30} \mathrm{O}_{4}$ : 528.2211. Found: 528.2217.

$(R, R, R)-30:{ }^{1} \mathrm{H}-\mathrm{NMR}\left(200 \mathrm{MHz}, \mathrm{CDCl}_{3}\right) \delta 0.58(\mathrm{t}, J=7.1,6 \mathrm{H}), 0.73(\mathrm{t}, J=6.2,6 \mathrm{H}), 0.9-1.05(\mathrm{~m}$, $12 \mathrm{H}), 2.5-2.8(\mathrm{~m}, 4 \mathrm{H}), 2.9-3.3(\mathrm{~m}, 12 \mathrm{H}), 3.80(\mathrm{~d}, J=145.0,6 \mathrm{H}), 4.3-4.9(\mathrm{~m}, 8 \mathrm{H}), 5.36(\mathrm{~s}, 4 \mathrm{H}), 7.1-7.5$ (m, 22H), 7.5-7.8 (m, 6H); HRMS (FAB) Calcd for ${ }^{12} \mathrm{C}_{78}{ }^{13} \mathrm{C}_{2} \mathrm{H}_{86} \mathrm{O}_{12} \mathrm{~N}_{4} \mathrm{Na}$ : 1319.6207. Found: 1319.6230.

$(R, S, R)-30:{ }^{1} \mathrm{H}-\mathrm{NMR}\left(200 \mathrm{MHz}, \mathrm{CDCl}_{3}\right) \delta 0.57(\mathrm{t}, J=7.2,6 \mathrm{H}), 0.64(\mathrm{t}, J=7.0,6 \mathrm{H}), 0.95(\mathrm{t}, J=7.2$, $12 \mathrm{H}), 2.5-3.3(\mathrm{~m}, 16 \mathrm{H}), 3.81(\mathrm{~d}, J=145.2,6 \mathrm{H}), 4.39(\mathrm{~d}, J=12.0,2 \mathrm{H}), 4.47(\mathrm{~d}, J=12.2,2 \mathrm{H}), 4.71(\mathrm{~d}, J$ $=12.2,2 \mathrm{H}), 4.76(\mathrm{~d}, J=12.0,2 \mathrm{H}), 5.38(\mathrm{~s}, 4 \mathrm{H}), 7.2-7.5(\mathrm{~m}, 22 \mathrm{H}), 7.5-7.7(\mathrm{~m}, 4 \mathrm{H}), 7.82(\mathrm{~d}, J=8.2$, $2 \mathrm{H})$, ; HRMS (FAB) Calcd for ${ }^{12} \mathrm{C}_{78}{ }^{13} \mathrm{C}_{2} \mathrm{H}_{87} \mathrm{O}_{12} \mathrm{~N}_{4}: 1297.6388$. Found: 1297.6373.

$(R, R, R, R, R, R, R)-31: \mathrm{Mp}, 132{ }^{\circ} \mathrm{C} ;[\alpha]_{\mathrm{D}}^{20}=+120\left(\mathrm{c}=0.50, \mathrm{CHCl}_{3}\right) ; \mathrm{IR}(\mathrm{KBr}) 2930,1642,1550,1446$ $\mathrm{cm}^{-1} ;{ }^{1} \mathrm{H}-\mathrm{NMR}\left(200 \mathrm{MHz}, \mathrm{CDCl}_{3}\right) \delta$ 0.5-1.2 (m, 48H), 2.6-3.4 (m, 32H), $3.84(\mathrm{~s}, 6 \mathrm{H}), 3.89(\mathrm{~s}, 6 \mathrm{H})$, $3.91(\mathrm{~d}, J=145 \mathrm{~Hz}, 6 \mathrm{H}), 4.4-5.1(\mathrm{~m}, 16 \mathrm{H}), 5.37(\mathrm{~s}, 4 \mathrm{H}), 7.1-7.7(\mathrm{~m}, 42 \mathrm{H}), 7.83(\mathrm{~d}, J=8.0 \mathrm{~Hz}, 2 \mathrm{H})$; MS (FAB) $m / z 2460(\mathrm{M}+\mathrm{Na})^{+}$ 
$(R, R, R, S, R, R, R)-31: \mathrm{Mp}, 130{ }^{\circ} \mathrm{C} ;[\delta]_{\mathrm{D}}{ }^{20}=+115\left(\mathrm{c}=0.35, \mathrm{CHCl}_{3}\right) ; \mathrm{IR}(\mathrm{KBr}) 2933,1648,1457,1396$ $\mathrm{cm}^{-1} ;{ }^{1} \mathrm{H}-\mathrm{NMR}\left(200 \mathrm{MHz}, \mathrm{CDCl}_{3}\right) \delta$ 0.5-1.3 (m, 48H), 2.6-3.4 (m, 32H), $3.89(\mathrm{~s}, 12 \mathrm{H}), 3.88(\mathrm{~d}, J=$ $144.8 \mathrm{~Hz}, 6 \mathrm{H}), 4.0-5.1(\mathrm{~m}, 16 \mathrm{H}), 5.37(\mathrm{~s}, 4 \mathrm{H}), 7.1-7.7(\mathrm{~m}, 42 \mathrm{H}), 7.83(\mathrm{~d}, J=8.2 \mathrm{~Hz}, 2 \mathrm{H}) ;$ MS (FAB) $m / z 2460(\mathrm{M}+\mathrm{Na})^{+}$ 

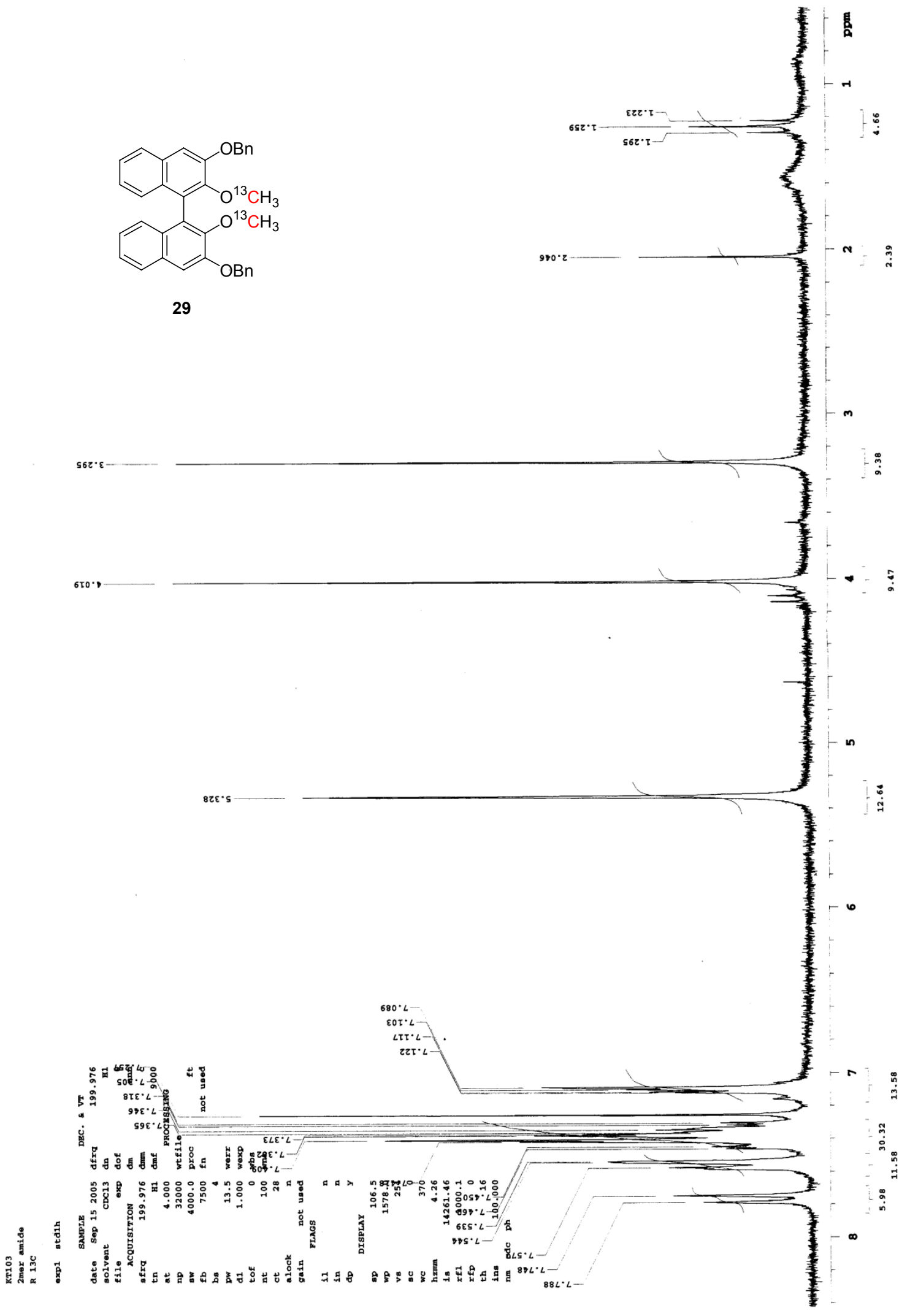


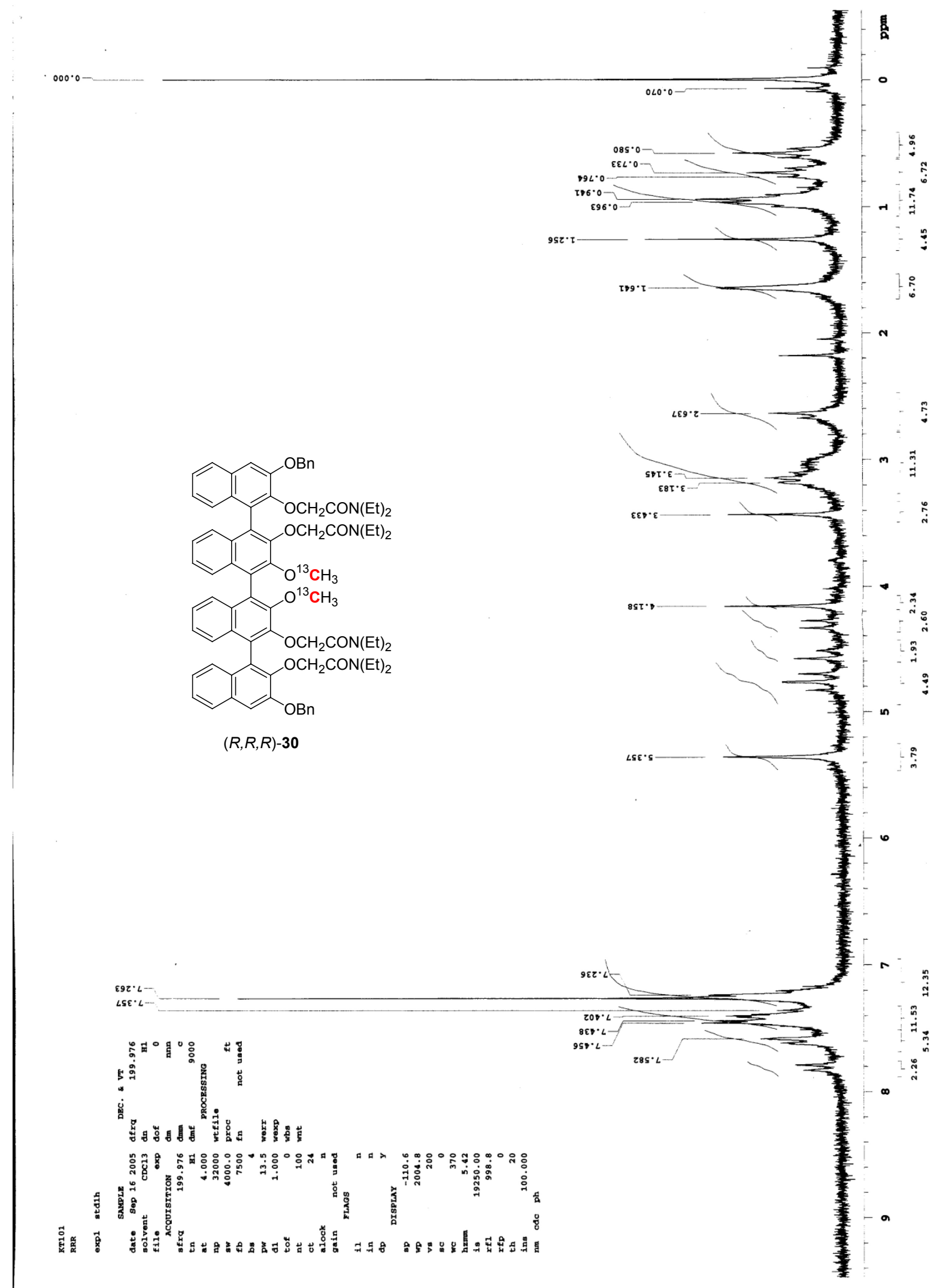




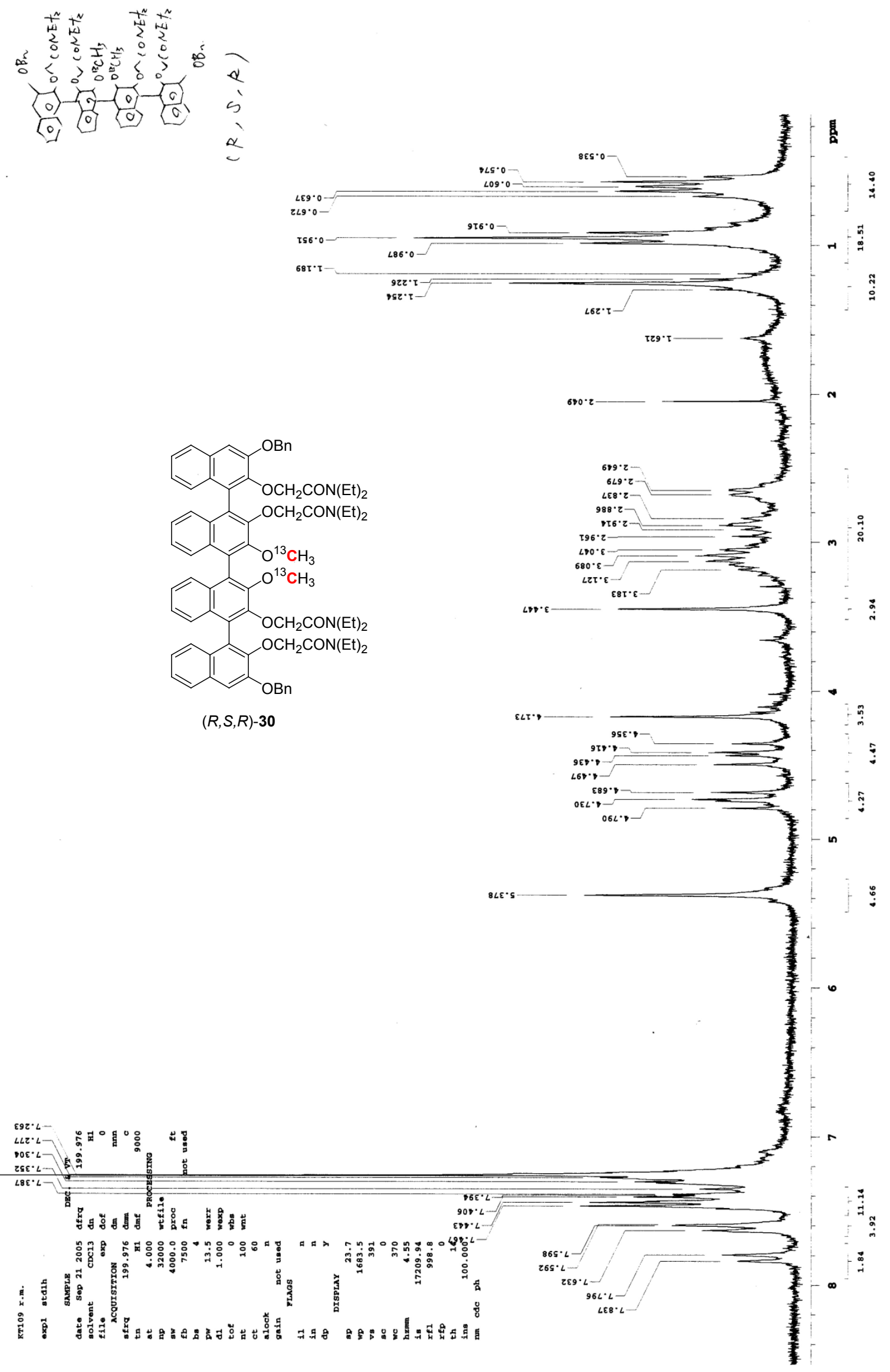




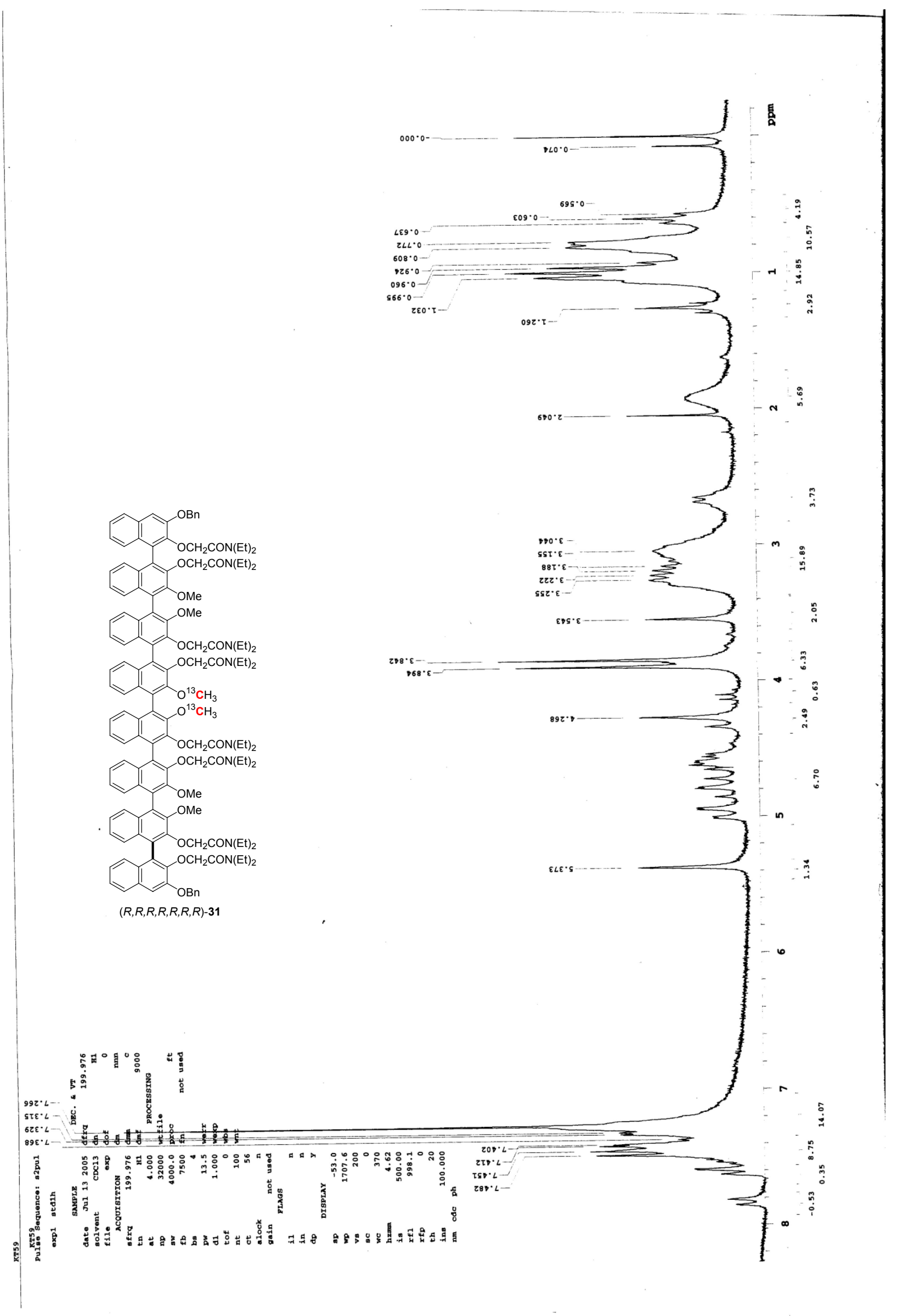




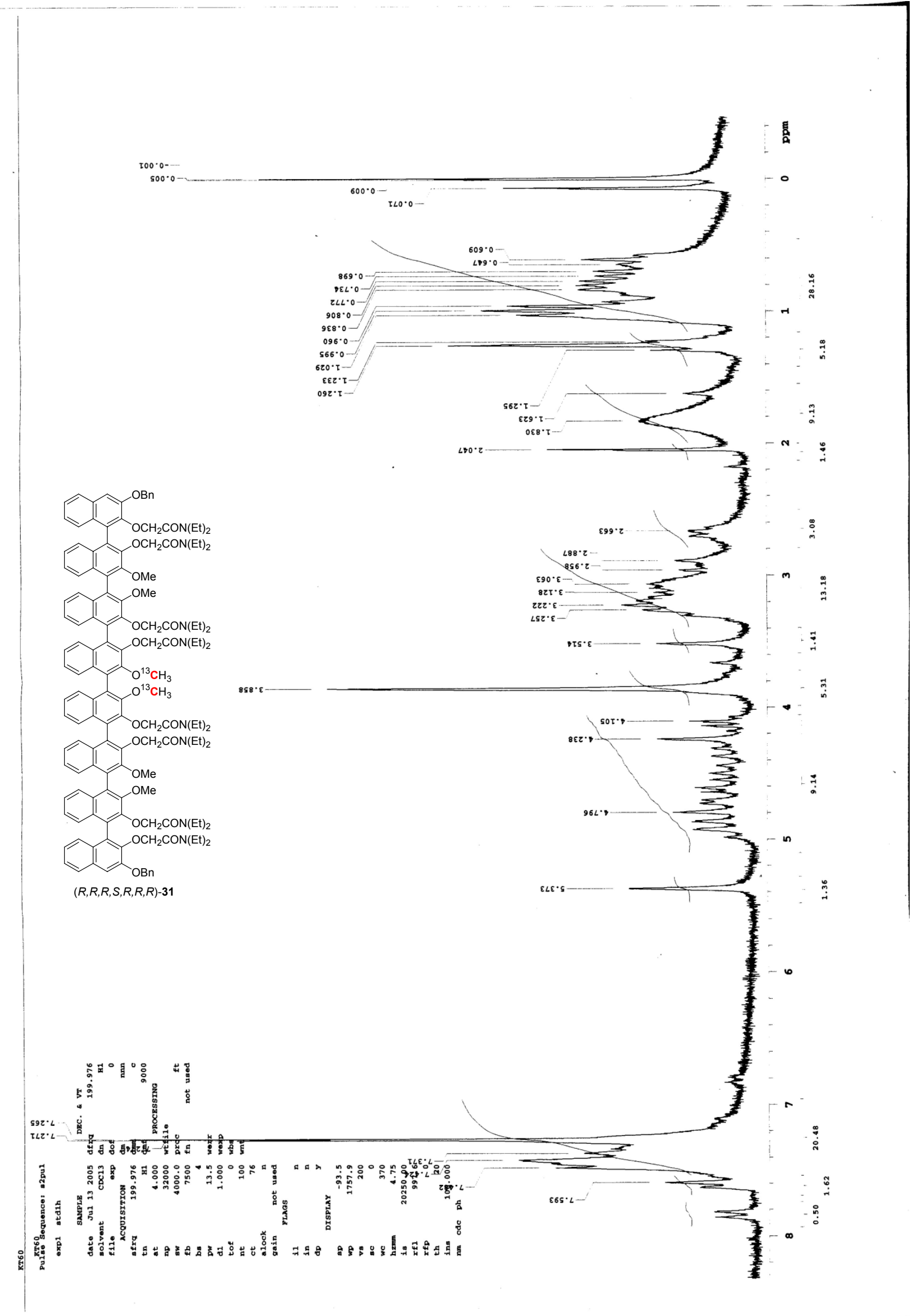

